\title{
VIBRATION ANALYSIS OF BEAMS USING ALTERNATIVE ADMISSIBLE FUNCTIONS WITH PENALTIES
}

\author{
Srividyadhare MC Kateel
}

\author{
A thesis submitted to the Faculty of Engineering \\ in partial fulfilment of the requirements for the degree of \\ MASTER OF APPLIED SCIENCE \\ in Mechanical Engineering \\ Ottawa-Carleton Institute for Mechanical and Aerospace Engineering \\ University of Ottawa \\ Ottawa, Canada \\ January 2022
}

(C) Srividyadhare M C Kateel, Ottawa, Canada, 2022 
Dedicated to my parents and grandmother, The most precious people I have in this world 


\section{Abstract}

Establishing dynamic characteristics of structures is a challenging area of research. The dynamic characteristics of structures, such as natural frequencies, modeshapes, response levels and damping characteristics play an important role in identifying the condition of the structures. The assumed modes method is a particular analytical method used to estimate the dynamic characteristics of a structure. However, the eigenfunctions used in the assumed mode method often led to illconditioning due to the presence of hyperbolic functions. Furthermore, a change in the boundary conditions of the system usually necessitates a change in the choice of assumed mode.

In this thesis, a set of Alternative Admissible Functions $(A A F)$, along with penalty functions, are used to obtain closed form solutions for an Euler-Bernoulli beam with various boundary conditions. A key advantage of the proposed approach is that the choice of $A A F$ does not depend on the boundary conditions since the boundary conditions are modelled via penalty functions. The mathematical formulation is validated with different boundary conditions, Clamped-Free $(C F)$, Simply-Supported $(S S)$, and Clamped-Clamped $(C C)$. A specific relation between the penalty function and the system parameters are established for $C F, S S$ and $C C$ boundary conditions to obtain appropriate values of penalties. Validation of results with the reported literature indicates excellent agreement when compared with closed-form Euler-Bernoulli beam values.

The $A A F$ approach with penalties is extended to a beam with a shallow crack to estimate the dynamic characteristics. The crack is modelled as a penalty function via a massless rotational spring. This model has the advantage of simplifying parametric studies, because of its discrete nature, allowing easy modification in the crack position and depth of the crack. Therefore, once the model is established, various practical applications may be performed without reformulation 
of the problem. Validation of results with the reported literature on beams with shallow cracks indicates the suitability of the proposed approach. 


\section{Acknowledgement}

My sincere gratitude to my wonderful supervisor, Dr. Natalie Baddour, for believing in my skills, providing me an excellent research opportunity, and for her constant support. As a role model, I look up to her as a source of great inspiration. She taught me more than just science that will guide me in my life. Your input, trust, and devotion made this thesis a success.

To Dr. Aneta Traikova for your incredible support and belief in me are invaluable! It is hard to find the words that can describe gratitude and appreciation towards you.

My friends and roommates have made these two years memorable, reminding me to have fun and put a smile on my face when I needed to.

Most of all, I would like to thank my mom and dad for always motivating me to thrive for the best. You guys are inspiration and your belief in me kept me going. For all the trust you had in me - I thank you! I would have never made it without you both! 


\section{Table of Contents}

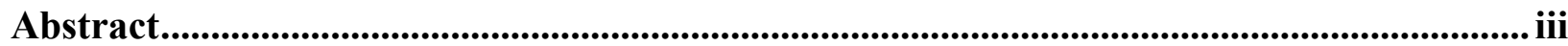

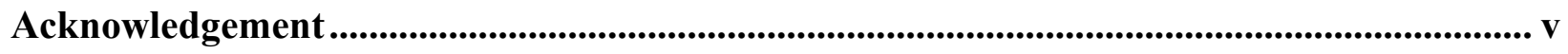

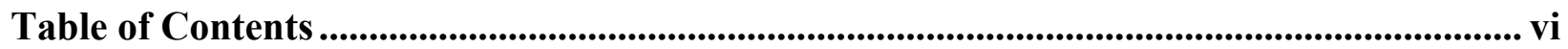

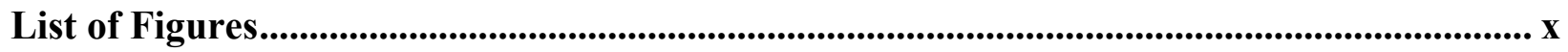

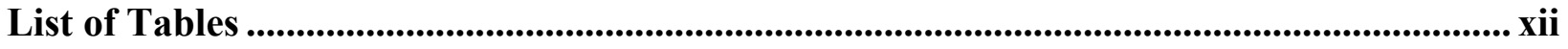

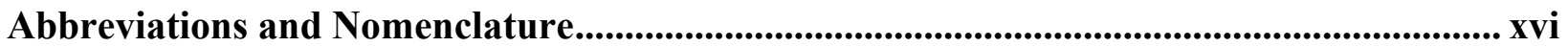

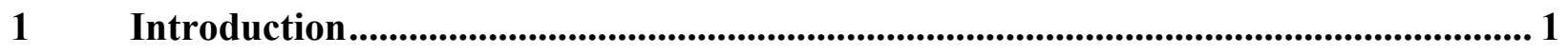

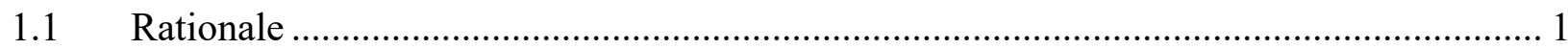

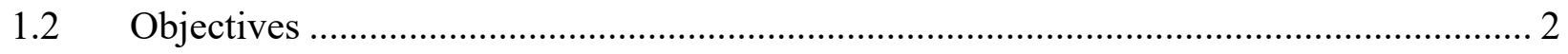

1.3 Thesis Contributions ............................................................................................. 3

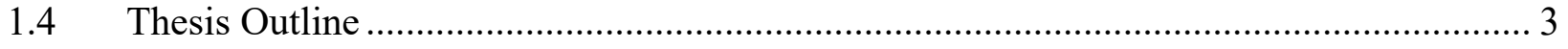

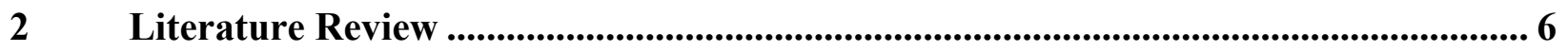

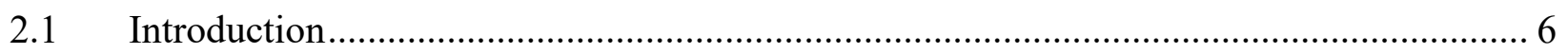

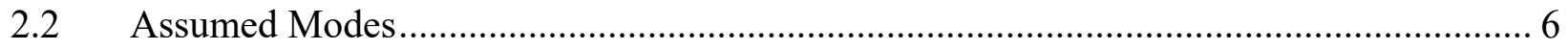

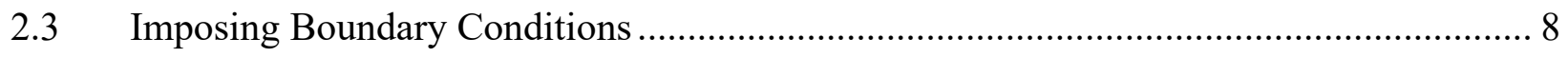

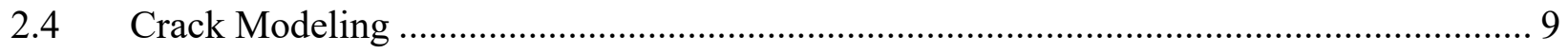

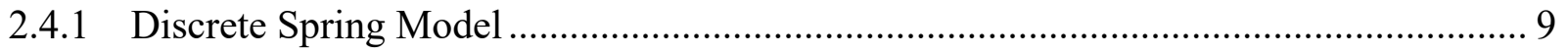

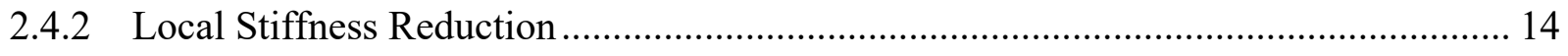




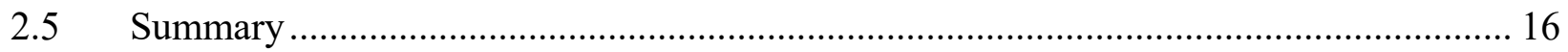

$3 \quad$ Mathematical Model using Alternative Admissible Functions....................................... 18

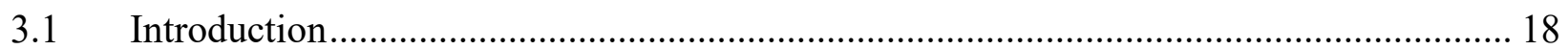

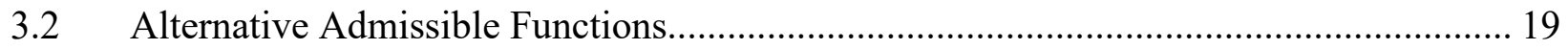

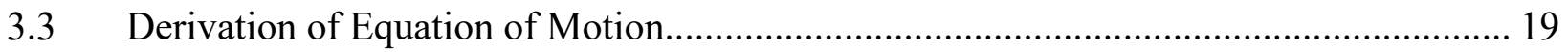

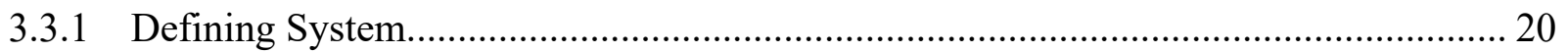

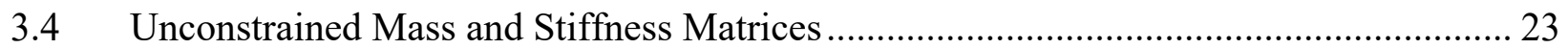

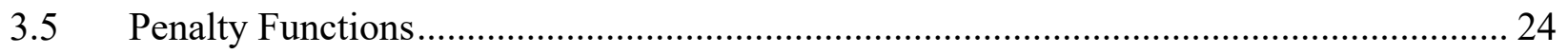

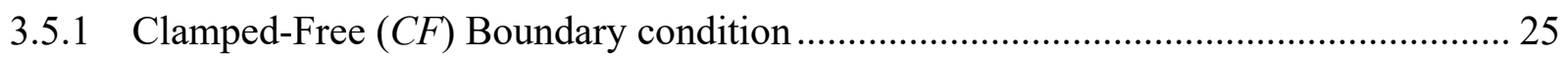

3.5.2 Simply-Supported (SS) Boundary condition........................................................... 26

3.5.3 Clamped-Clamped (CC) Boundary condition ………........................................... 27

3.6 Penalty Stiffness Matrix for Different Boundary Conditions ....................................... 28

3.6.1 Penalty Stiffness Matrix for Clamped-Free $(\mathrm{CF})$ Condition ....................................... 28

3.6.2 Penalty Stiffness Matrix for Simply-Supported (SS) Condition ................................... 30

3.6.3 Penalty Stiffness Matrix for Clamped-Clamped (CC) Condition................................. 32

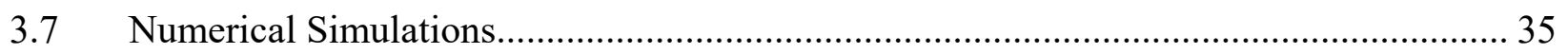

3.7.1 Geometrical Parameters and Material Characteristics ................................................. 35

3.7.2 Numerical Example on Clamped-Free $(C F)$ Beam ………………………............. 36

3.7.3 Numerical Example on Simply-Supported (SS) Beam ............................................... 39

3.7.4 Numerical Example on Clamped-Clamped (CC) Beam ............................................... 42 
3.8 Numerical Example on Varying Thickness for All Three Boundary Conditions. 45

3.9 Conclusions 50

4 Selection and Validation of Penalty Values for Alternative Admissible Function ... 51

4.1 Introduction. 51

4.2 Penalty Function for Different Boundary Conditions............................................... 52

4.2.1 Penalty Function for Clamped-Free $(C F)$ Boundary Condition ................................ 54

4.2.2 Penalty Function for Simply-Supported (SS) Boundary Condition........................... 57

4.2.3 Penalty Function for Clamped-Clamped $(C C)$ Boundary Condition.......................... 59

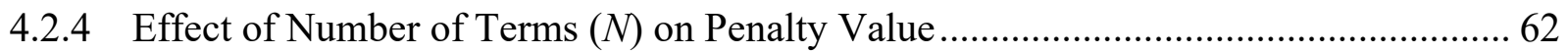

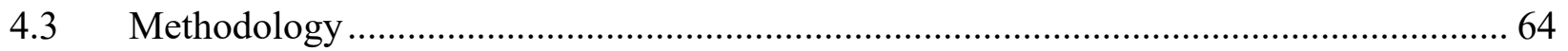

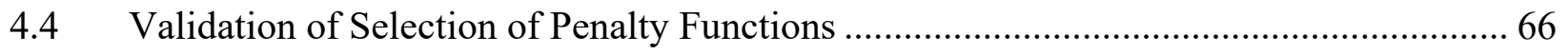

4.4.1 Geometrical Parameters and Material Characteristics .......................................... 66

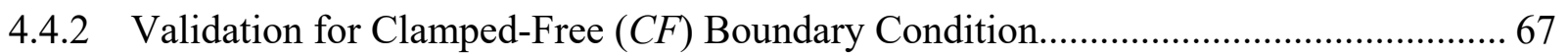

4.4.3 Validation for Simply-Supported (SS) Boundary Condition ................................... 73

4.4.4 Validation for Clamped-Clamped $(C C)$ Boundary Condition ................................... 78

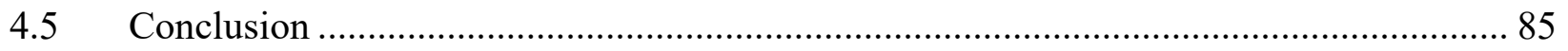

5 Alternative Admissible Function Approach for a Beam with a Shallow Crack ....... 86

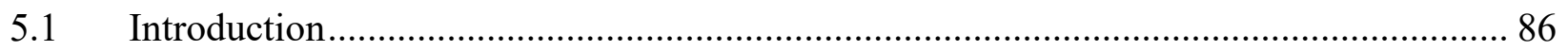

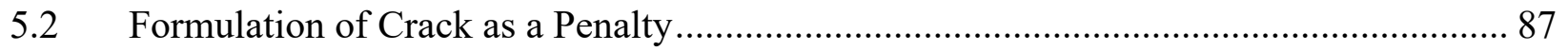

5.3 Equation of Motion of a Beam with a Shallow Crack ........................................... 90 


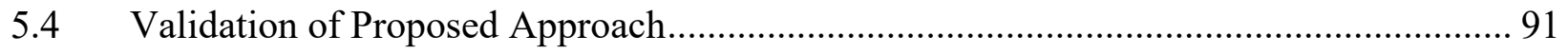

5.4.1 Crack Parameters .......................................................................................... 91

5.4.2 Validation of a Shallow Crack in a Simply-Supported (SS) Beam........................... 91

5.4.3 Validation of a Shallow Crack in a Clamped-Clamped $(C C)$ Beam ......................... 95

5.4.4 Validation of a Shallow Crack in a Clamped-Free $(C F)$ Beam ............................... 98

5.4.5 Validation of Proposed Approach to Estimate Modeshapes.................................... 102

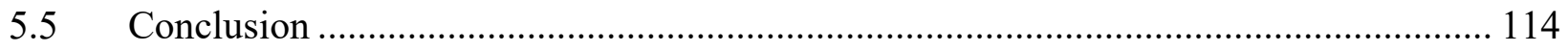

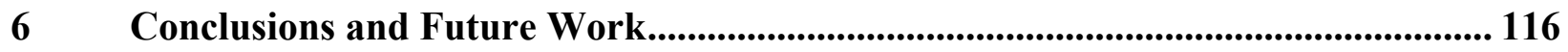

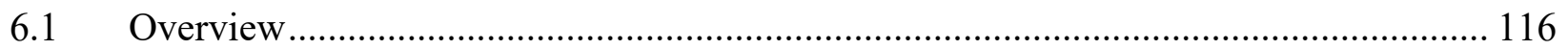

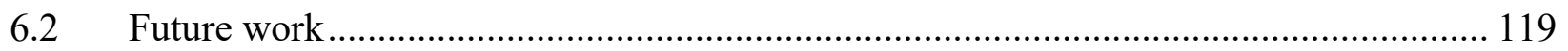

References................................................................................................................................................ 120 


\section{List of Figures}

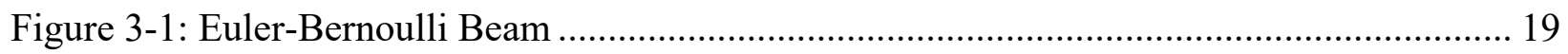

Figure 3-2: First Five Normalized Alternative Admissible Functions ......................................... 21

Figure 3-3: Beam with Clamped-Free $(C F)$ Boundary Condition............................................. 25

Figure 3-4: Beam with Simply-Supported (SS) Boundary Condition ....................................... 26

Figure 3-5: Beam with Clamped-Clamped $(C C)$ Boundary Condition ...................................... 27

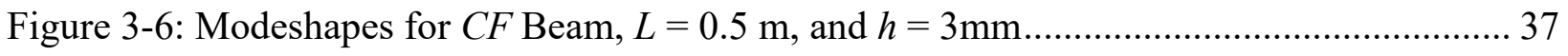

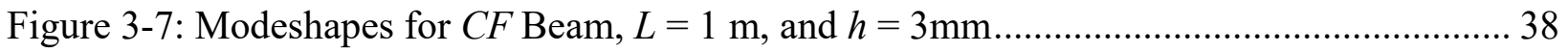

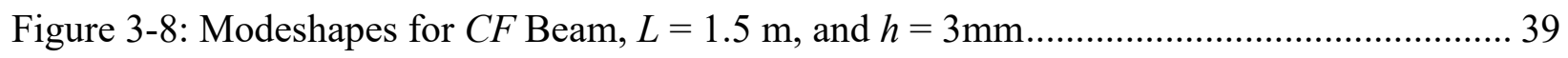

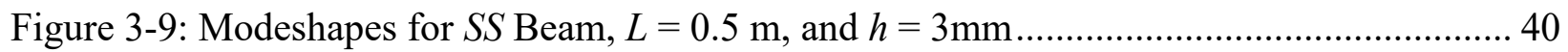

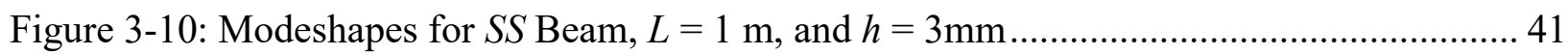

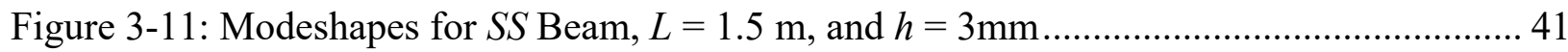

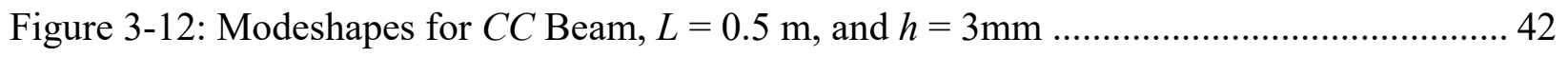

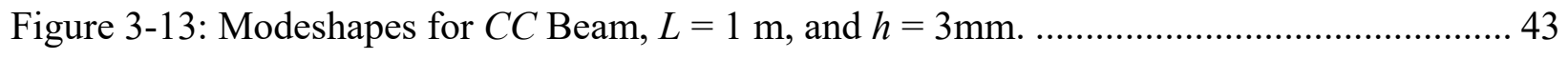

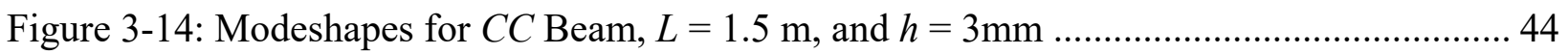

Figure 3-15: Deviation v/s Model Number for Various Penalty Values ...................................... 49

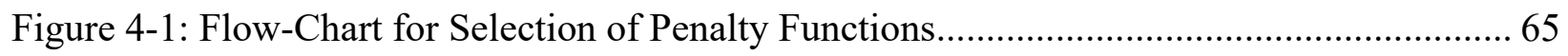

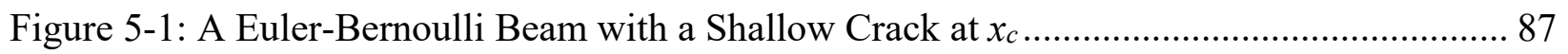

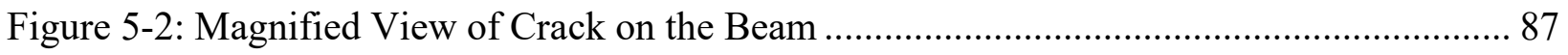

Figure 5-3: Crack Represented as a Massless Rotational Spring ................................................. 88

Figure 5-4: Modeshapes of SS Beam with Crack Depth Ratio, $(\mathrm{dc} / \mathrm{h})=0.2$. A. Normalized Location of Crack, $(x c)=0.2$, B. Normalized Location of Crack, $(x c)=0.8$, C. Normalized Location of Crack, $(x c)=0.4$, and D. Normalized Location of Crack, $(x c)=0.6 \ldots \ldots \ldots \ldots \ldots \ldots . . .104$ 
Figure 5-5: Modeshapes of SS Beam with Crack Depth Ratio, $\left(d_{c} / h\right)=0.4$, A. Normalized Location of Crack, $\left(x_{c}\right)=0.2$, B. Normalized Location of Crack, $\left(x_{c}\right)=0.8$, C. Normalized Location of Crack, $\left(x_{c}\right)=0.4$, and D. Normalized Location of Crack, $\left(x_{c}\right)=0.6$ 106

Figure 5-6: Modeshapes of CC Beam with Crack Depth Ratio, $(d c / h)=0.2$, A. Normalized Location of Crack, $(\mathrm{xc})=0.2$, B. Normalized Location of Crack, $\left(x_{c}\right)=0.8$, C. Normalized Location of Crack, $\left(x_{c}\right)=0.4$, and D. Normalized Location of Crack, $\left(x_{c}\right)=0.6$. 108 Figure 5-7: Modeshapes of $C C$ Beam with Crack Depth Ratio, $\left(d_{c} / h\right)=0.4$. A. Normalized Location of Crack, $(\mathrm{xc})=0.2$, B. Normalized Location of Crack, $\left(x_{c}\right)=0.8$, C. Normalized Location of Crack, $\left(x_{c}\right)=0.4$, and D. Normalized Location of Crack, $\left(x_{c}\right)=0.6$. 111 Figure 5-8: Modeshapes of $C F$ Beam with Crack Depth Ratio, $(d c / h)=0.5$ 113 


\section{List of Tables}

Table 3-1: Admissible Functions and 1st and 2nd Derivative............................................... 23

Table 3-2: Clamped-Free (CF) First Part of Eq.(3.15) ......................................................... 29

Table 3-3: Clamped- Free $(C F)$ Second Part of Eq.(3.15).................................................. 29

Table 3-4: Simply-Supported (SS) First Part of Eq.(3.16) .................................................. 30

Table 3-5: Simply-Supported (SS) Second Part of Eq.(3.16) ............................................. 31

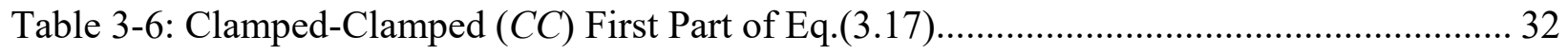

Table 3-7: Clamped-Clamped (CC) Second Part of Eq.(3.17) .............................................. 33

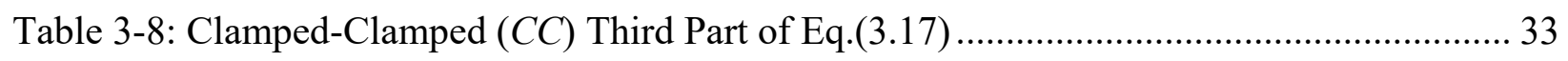

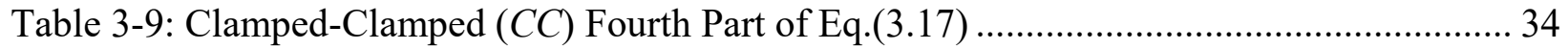

Table 3-10: Geometrical Parameters \& Material Characteristics ............................................ 35

Table 3-11: Natural Frequencies: $C F$ Beam, $L=0.5 \mathrm{~m}$, and $h=3 \mathrm{~mm}$.................................. 37

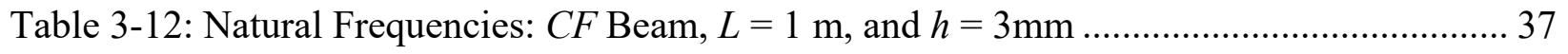

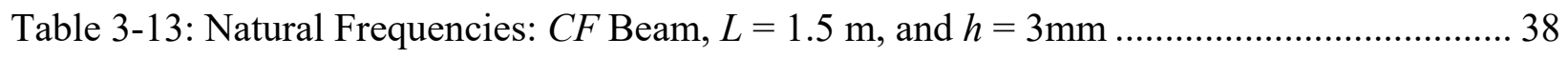

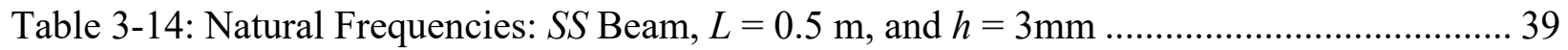

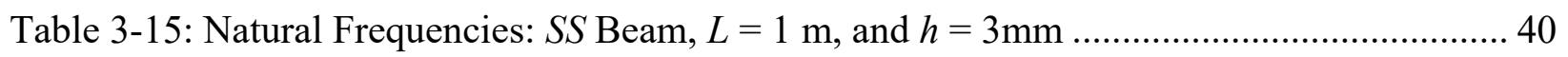

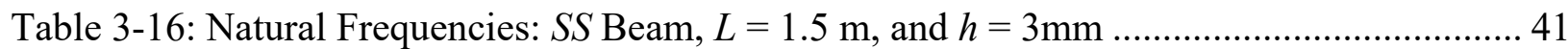

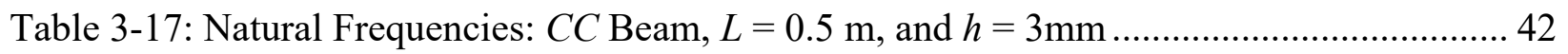

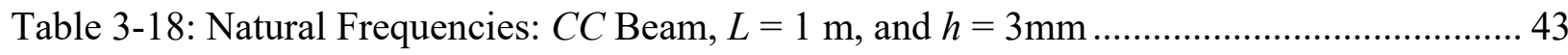

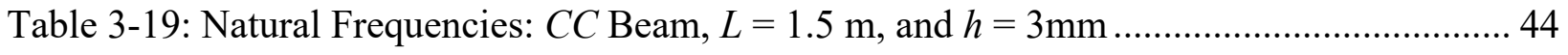

Table 3-20: Natural Frequencies: $C F$ Beam, $L=1 \mathrm{~m}$, and $h=3 \mathrm{~mm}$ and $4 \mathrm{~mm} \ldots \ldots \ldots \ldots \ldots \ldots \ldots . . . . . . . . . .45$

Table 3-21: Natural Frequencies: CF Beam, $L=1 \mathrm{~m}$, and $h=5 \mathrm{~mm}$................................. 46

Table 3-22: Natural Frequencies: $C C$ Beam, $L=1 \mathrm{~m}$, and Varying Thickness $(h) \ldots \ldots \ldots \ldots \ldots \ldots . . . . . . . . .47$ 
Table 3-23: Natural Frequencies: $S S$ Beam, $L=1 \mathrm{~m}$, and Varying Thickness $(h) \ldots \ldots \ldots \ldots \ldots \ldots \ldots . . . . . . . .48$

Table 3-24: Deviations in Predicted Natural Frequencies $(H z)$ with Varying Penalty Value ...... 49

Table 4-1: $k_{1}$ and $k_{2}$ Values for $C F$ Boundary Condition ............................................... 56

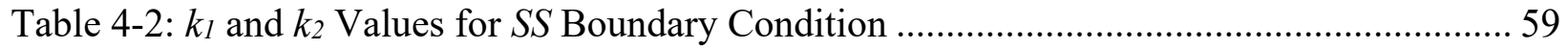

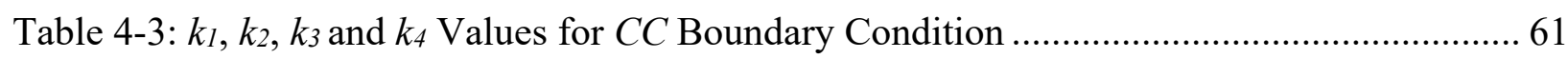

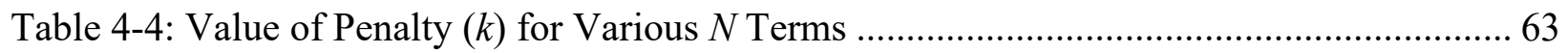

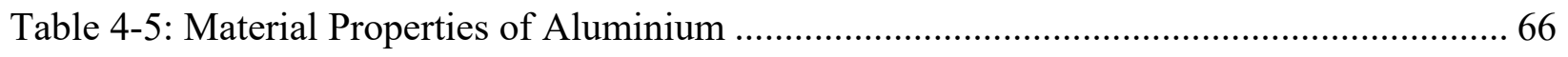

Table 4-6: Calculated Values of Penalty Function $C F$ Boundary Condition ............................. 67

Table 4-7: Natural Frequencies of $C F$ Beam with $L=0.5 \mathrm{~m}$ and $h=3 \mathrm{~mm}$. The $k 0=1 \mathrm{X} 10^{10}$ value is from the literature and the $k 0=1 \times 10^{7.29}$ value is from the proposed methodology............... 68

Table 4-8: Natural Frequencies of $C F$ Beam with $L=1.0 \mathrm{~m}$ and $h=3 \mathrm{~mm}$............................ 69

Table 4-9: Natural Frequencies of $C F$ Beam with $L=1.5 \mathrm{~m}$ and $h=3 \mathrm{~mm}$.......................... 69

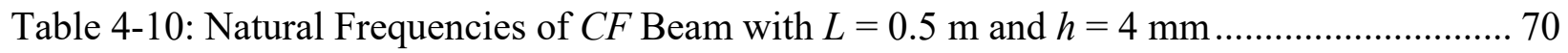

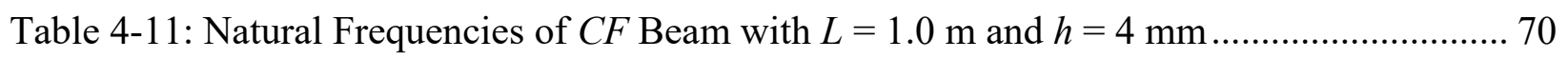

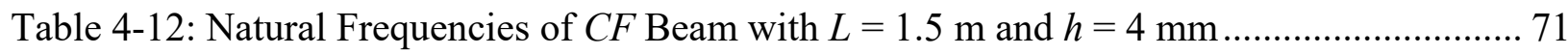

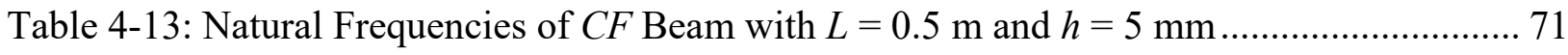

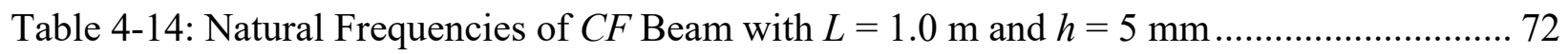

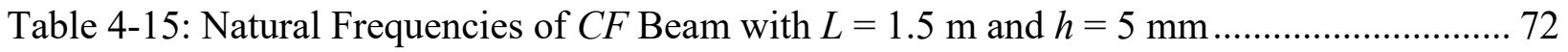

Table 4-16: Calculated Values of Penalty Function for SS Boundary Conditions ..................... 73

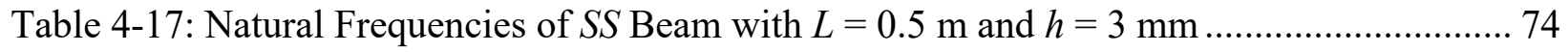

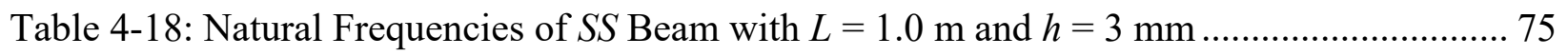

Table 4-19: Natural Frequencies of SS Beam with $L=1.5 \mathrm{~m}$ and $h=3 \mathrm{~mm}$......................... 75

Table 4-20: Natural Frequencies of SS Beam with $L=0.5 \mathrm{~m}$ and $h=4 \mathrm{~mm}$.......................... 76 
Table 4-21: Natural Frequencies of SS Beam with $L=1.0 \mathrm{~m}$ and $h=4 \mathrm{~mm}$.............................. 76

Table 4-22: Natural Frequencies of $S S$ Beam with $L=1.5 \mathrm{~m}$ and $h=4 \mathrm{~mm}$............................. 77

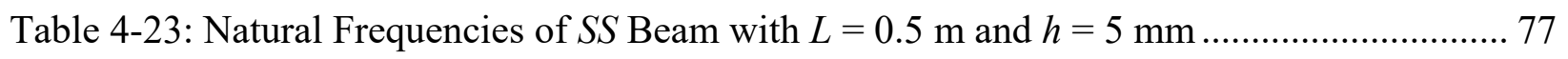

Table 4-24: Natural Frequencies of SS Beam with $L=1.0 \mathrm{~m}$ and $h=5 \mathrm{~mm}$.............................. 78

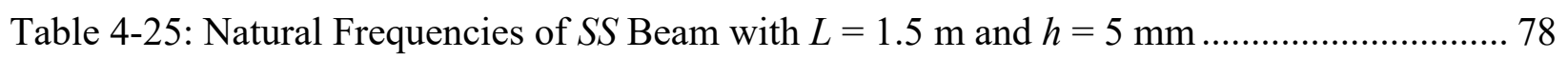

Table 4-26: Calculated Values of Penalty Function for $C C$ Boundary Conditions ....................... 79

Table 4-27: Natural Frequencies of $C C$ Beam with $L=0.5 \mathrm{~m}$ and $h=3 \mathrm{~mm}$............................. 80

Table 4-28: Natural Frequencies of $C C$ Beam with $L=1.0 \mathrm{~m}$ and $h=3 \mathrm{~mm}$............................. 80

Table 4-29: Natural Frequencies of $C C$ Beam with $L=1.5 \mathrm{~m}$ and $h=3 \mathrm{~mm}$............................. 81

Table 4-30: Natural Frequencies of $C C$ Beam with $L=0.5 \mathrm{~m}$ and $h=4 \mathrm{~mm}$............................. 81

Table 4-31: Natural Frequencies of CC Beam with $\mathrm{L}=1.0 \mathrm{~m}$ and $\mathrm{h}=4 \mathrm{~mm}$............................ 82

Table 4-32: Natural Frequencies of $C C$ Beam with $L=1.5 \mathrm{~m}$ and $h=4 \mathrm{~mm}$............................ 82

Table 4-33: Natural Frequencies of $C C$ Beam with $L=0.5 \mathrm{~m}$ and $h=5 \mathrm{~mm}$............................ 83

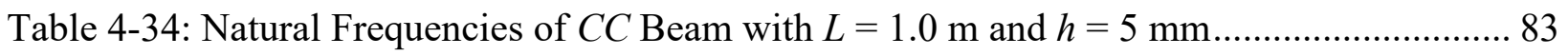

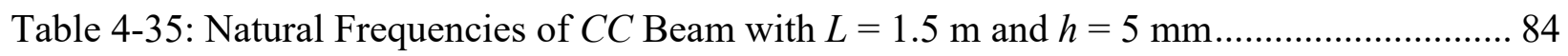

Table 5-1: Geometrical Parameters and Material Properties ....................................................... 91

Table 5-2: Natural Frequencies $(\mathrm{Hz})$ of SS Beam with Crack Depth Ratio, $\left(d_{c} / h\right)=0.2 \ldots \ldots \ldots \ldots . . .93$

Table 5-3: Natural Frequencies $(\mathrm{Hz})$ of $S S$ Beam with Crack Depth Ratio, $\left(d_{c} / h\right)=0.4$............ 94

Table 5-4: Natural Frequencies $(\mathrm{Hz})$ of $C C$ Beam with Crack Depth Ratio, $\left(d_{c} / h\right)=0.2 \ldots \ldots \ldots . . . .96$

Table 5-5: Natural Frequencies (Hz) of $C C$ Beam with Crack Depth Ratio, $\left(d_{c} / h\right)=0.4 \ldots \ldots \ldots \ldots . . .97$

Table 5-6: Geometrical Parameters and Material Properties Khnaijar and Benamar [18] ........... 98

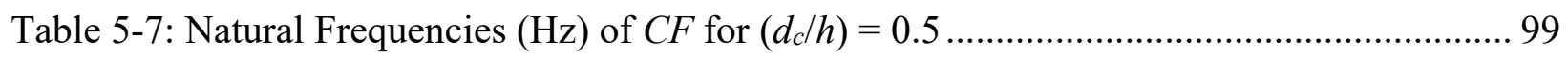

Table 5-8: Geometrical Parameters and Material Properties Barad et.al., [20]......................... 100 


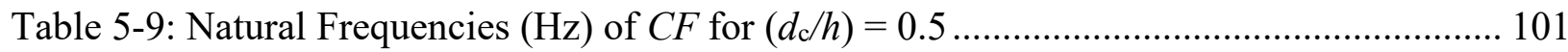




\section{Abbreviations and Nomenclature}

$A A F \quad$ Alternative Admissible Functions

$E B B \quad$ Euler-Bernoulli Beam

$N \quad$ Number of terms

$R R M \quad$ Rayleigh-Ritz Method

$w(x, t) \quad$ Transverse Response

$W(x) \quad$ Amplitude of Vibration

$\omega \quad$ Frequency of Vibration

$\phi_{i}(x) \quad$ Set of Assumed Modes

$k_{0} \quad$ Virtual Linear Virtual Spring

$k_{r 0} \quad$ Virtual Rotational Spring

$k_{c} \quad$ Virtual Massless-Rotational Spring

$T_{\max } \quad$ Maximum Kinetic Energy

$V_{\max } \quad$ Maximum Potential Energy

$V_{p} \quad$ Potential Energy of Penalty Terms

$V_{c} \quad$ Potential Energy due to the Presence of Crack

$V_{\text {mod }} \quad$ Modified Potential Energy of Beam with Crack

$m_{i j} \quad i^{\text {th }}$ and $j^{\text {th }}$ Entries of the Mass Matrix

$k_{i j} \quad i^{\text {th }}$ and $j^{\text {th }}$ Entries of the Stiffness Matrix

$k_{P, i j} \quad i^{\text {th }}$ and $j^{\text {th }}$ Entries of the Penalty Matrix

$k_{P, C F i j} \quad i^{\text {th }}$ and $j^{\text {th }}$ Entries of the Clamped-Free Boundary Condition Penalty 


$\begin{array}{ll}k_{P, S S i j} & \begin{array}{l}i^{\text {th }} \text { and } j^{\text {th }} \text { Entries of the Simply-Supported Boundary Condition Penalty } \\ \end{array} \\ & \text { Matrix } \\ k_{P, C C i j} & i^{\text {th }} \text { and } j^{\text {th }} \text { Entries of the Clamped-Clamped Boundary Condition } \\ & \text { Penalty Matrix } \\ k_{C, i j} & i^{\text {th }} \text { and } j^{\text {th }} \text { Entries of the Cracked Beam Portion Matrix } \\ k_{E, i j} & i^{\text {th }} \text { and } j^{\text {th }} \text { Entries of Effective Stiffness of a Shallow Cracked Beam } \\ M & \quad \text { Mass Matrix } \\ K & \quad \text { Stiffness Matrix } \\ K_{P, C F} & \text { Penalty Matrix for Clamped-Free Boundary Condition } \\ K_{P, S S} & \text { Penalty Matrix for Simply-Supported Boundary Condition } \\ K_{P, C C} & \text { Penalty Matrix for Clamped-Clamped Boundary Condition } \\ K_{T} & \text { Total Stiffness Matrix } \\ K_{C} & \text { Stiffness Matrix of Cracked Beam Portion } \\ K_{E} & \text { Effective Stiffness of a Shallow Cracked Beam }\end{array}$




\section{Introduction}

\subsection{Rationale}

Structural dynamics has been a challenging field of research for decades. Establishing accurate eigenvalues and eigenvectors are one of the basic keys for the success of dynamic structural analysis of continuous structures. One of the continuous structural elements of interest is beams. Different methods, approaches, and procedures have been established to estimate the natural frequencies and modeshapes of beams.

The assumed modes method [1] is one such approach, established and often used for many traditional boundary conditions. The trial functions used in an assumed mode approach are typically chosen to satisfy both the natural and geometrical boundary conditions. Changes in the boundary condition usually necessitates a change in the trial functions. Typically, to assemble the Rayleigh quotient, the chosen functions must be twice differentiable. The additional differentiation usually leads to difficulty in computation and an increase in computational time. Additionally, if the chosen functions are hyperbolic sines and cosines, such functions often lead to numerical instability [2].

On the other hand, Alternative Admissible Functions $(A A F)$ use a set of lower-order polynomials and trigonometric functions that represent an unconstrained beam [2]. The functions are selected to obtain the simplest set of functions that converge quickly and do not have a limitation on the number of terms due to numerical instability. The boundary conditions are then implemented on the unconstrained beam using penalty functions. A combination of virtual linear and rotational springs at the boundaries constitutes penalty functions. The potential energy associated with virtual linear and rotational spring, are calculated to implement different boundary conditions in the system. 
The present work focuses on establishing a specific value of penalty functions for $A A F$ method depending on the different beam parameters and boundary conditions. A generalized mathematical procedure for the process of establishing the expression is proposed and validated. Additionally, this thesis presents the analysis of a beam with a shallow crack using the $A A F$ approach, where the shallow crack is implemented as a penalty function. Estimated boundary penalty values with the crack penalty model are illustrated with numerical examples. The obtained results are validated with the reported literature.

The assumed modes method is improved by using a set of $A A F$. The $A A F$ combined with penalty functions provides flexibility with changes in boundary conditions. An improved convergence is obtained by using specific values for the boundary penalty functions. For cracked beams, the crack is modelled as a penalty and provides flexibility to relocate the crack at different locations and different depths. The proposed method using $A A F$ with penalty functions provides future scope for analytical solutions beyond the traditional boundary conditions. The established mathematical procedure is a promising platform for obtaining the natural frequencies and modeshapes for various complex and built-up structures.

\subsection{Objectives}

A set of targeted objectives is set for the present work and is as follows:

- To develop a penalty stiffness matrix for an Euler Bernoulli beam with several traditional boundary conditions viz., Clamped-Free $(C F)$, Simply-Supported (SS) and Clamped-Clamped $(C C)$ boundary conditions, which do not exist in the literature to date. 
- To propose a method to estimate the value of the penalty functions for use with the AAF approach, and to validate the proposed approach with benchmark closed-form EulerBernoulli beam values.

- To extend the use of $A A F$ approach with penalty functions to Euler-Bernoulli beams with a shallow crack and validate the results with reported literature.

\subsection{Thesis Contributions}

The research focuses on the estimation of natural frequencies and modes shapes of an EulerBernoulli beam using Alternative Admissible Functions $(A A F)$. Generalized mass and stiffness matrices for the unconstrained beam are successfully established using the set of functions. The boundary conditions are implemented via Penalty Functions. Penalty stiffness matrices are established for three classical boundary conditions viz., Clamped-Free $(C F)$, Simply-Supported (SS), and Clamped-Clamped (CC) boundary conditions. The validation of the $A F F$ is carried out for the aforementioned boundary conditions with closed-form Euler Bernoulli Beam values.

A relationship for estimating a specific penalty function value for different boundary conditions is established. Using the estimated values of penalty, the eigenvalues of the beam are validated with closed-form Euler Bernoulli beam values.

The AAF method along with the proposed methodology for estimating the penalty function values is applied to Euler-Bernoulli beams with shallow cracks where the crack is modelled as a penalty function. This novel approach to modelling the crack as a penalty function is then validated with reported literature values.

\subsection{Thesis Outline}

The present work is outlined into six chapters. The chapters outlined are as follows: 


\section{Chapter 2: Literature review}

Chapter 2 reports the literature review carried out and is divided into three sections. The first section starts with literature on the various trial functions used in modeling beams using the assumed modes method. The second section focuses on the literature related to penalty functions and existing values of penalties used in the analysis. Finally, the third section reports on the literature on beams with cracks. The various crack modeling methods are summarized. One such model is used as the basis for demonstrating the proposed methodology on beams with a crack.

\section{Chapter 3: Mathematical Model of Alternative Admissible Function}

Analytical approaches often make uses of true eigenfunctions or trial functions, which can often involve hyperbolic functions whose behaviors are numerically unstable. In this chapter, the use of Alternative Admissible Functions $(A A F)$ for the calculation of system natural frequencies and modeshapes is introduced and demonstrated. These $A A F$ are numerically stable and do not display the ill-conditioning that can occur when the system eigenfunctions are used. The detailed procedure used in estimating the natural frequencies and modeshapes using the Alternative Admissible Function is presented. The developed model is demonstrated using three different commonly-used boundary conditions, viz., Clamped-Free $(C F)$, Simply-Supported (SS) and Clamped-Clamped $(C C)$ boundary conditions. Numerical examples are validated using reported penalty values and compared with closed-form Euler-Bernoulli beam values as a benchmark. Chapter 3 concludes with the observation that an apparent relation exists between the penalty stiffnesses and the stiffness of the beam. 


\section{Chapter 4: Selection and Validation of Penalty Functions for Alternative Admissible Function}

Chapter 4 reports a comprehensive methodology in the selection of penalty values depending on the underlying beam properties and boundary conditions. The value of the penalties not only depends on the type of boundary conditions, but there is an apparent relation between the penalty function and the system parameters. An attempt is made to establish a general relation between the penalty functions and the system parameters. Validation of the proposed method is demonstrated with numerical examples and compared with closed-form Euler-Bernoulli beam values.

\section{Chapter 5: Alternative Admissible Function Approach for Shallow Cracked Beams}

This chapter starts with procedure for the formulation of a crack using a penalty function approach. The equation of motion for an Euler Bernoulli beam with a shallow crack is derived. Adoption of penalty functions for a shallow cracked beam is reported for three boundary conditions and the results are validated with reported literature.

\section{Chapter 6: Conclusions and Scope for Future Work}

Each chapter concludes with a brief note on the finding in the respective chapters as a set of conclusions. This chapter consolidates the conclusion for the entire thesis and highlights the scope for future work. 


\section{Literature Review}

\subsection{Introduction}

A thorough literature review is carried out covering the various aspects of vibration analysis of beams and beams with cracks. One of the important steps for the vibration analysis is the mathematical modeling of beam, various trial functions, comparison functions, trigonometric and hyperbolic functions are covered in the literature review. Different techniques in imposing the boundary conditions reported by many scholars are reviewed.

Modeling cracks in a beam for vibration analysis is a challenging task. Many researchers have attempted to model the crack in a beam using different approaches. In this chapter the literature review presented are under three different sub-headings covering assumed modes, imposing boundary conditions and crack modeling.

\subsection{Assumed Modes}

Meirovitch [1] presented a modern treatment for vibration analysis and developed various mathematical approaches emphasizing analytical and computational solutions. Presented solutions involving comparison functions, a linear combination of trial functions, and assumed-modes method etc. The Assumed-modes approach is elaborated in obtaining the natural frequencies and modeshapes. Monterrubio and Ilanko [2],[3] presented a detailed discussion on the characteristics of sets of admissible functions which may be used in the Rayleigh-Ritz method (RRM). They proposed sets of admissible functions and studied the convergence of results when penalty function terms are added to the model constraints. The proposed set of functions do not lead to illconditioning while handling large number of terms. Brown and stone [4] used a special set of polynomials to obtain the Rayliegh-Ritz solution for a laminated plate. They considered simply supported and clamped-clamped plates where all edge boundary conditions were considered. It 
was shown analytically that the selected set of polynomials does not affect the convergence of solution directly. Their results indicated that the set of polynomials are independent based on construction but are strictly a function of degree of polynomial in use. A review by Gorman and $\mathrm{Yu}[5]$ presented a relatively new method of structural analysis using the method of superposition developed for free vibration analysis of rectangular plates with various edge conditions. They concluded with a scope for future work in exploiting the proposed method for various other type of structures. Kim et.al., [6] proposed a set of simple polynomials for use as admissible functions in the RRM, for the study of the flexural vibration of rectangular plates. Applicability of the method and accuracy were demonstrated in presenting several numerical results. Other sets of admissible functions constructed from orthogonal polynomials using the Gram-Schmidt process were presented by Bhat [7] and shown to give excellent results for plates involving free edges.

Jaworski and Dowell [8] used trigonometric and hyperbolic functions to solve vibration problems of beams using a set of functions for the clamped-free boundary conditions of beams and reported that numerical problems arise due to the differences between the values of the hyperbolic functions. Li [9],[10] built a series of admissible functions by combining polynomials and trigonometric functions. It was stated that the polynomials are introduced to take care of relevant discontinuities with the original displacement and its derivatives at the boundaries.

Hosseini and Baddour [11] used the set of functions developed by [3] with the penalty function method. Boundary conditions were enforced using Penalty terms. They investigated the effects of adding lumped attachments to the structure on the effectiveness of the method. Several numerical results were presented for various boundary conditions. 


\subsection{Imposing Boundary Conditions}

Ahmadian et.al. [12] developed a new method to determine the boundary parameters based on the solution of reduced order characteristic equations of a plate. The boundary conditions were introduced to the model by adding the support stiffness to the stiffness matrix of the model and constraining the boundary motion by applying reaction forces at the boundary nodes. It was shown that such an approach does not lead to any ill-conditioning in the solutions. Roncevic et.al.,[13] considered free vibration analysis of an Euler-Bernoulli beam supported with an arbitrary number of translational springs of different constant stiffnesses. Green's function method used to estimate the natural frequencies and modeshapes. Several numerical results were obtained and validated with both reported literature and finite element method results.

Majkut [14] demonstrated the possible identification of boundary conditions in an ill-posed problem associated with natural frequencies. They developed a mathematical model for an EulerBernoulli beam validated with results obtained by analytical and numerical models. Chesne [15] illustrated the identification of boundary conditions for beams. The boundary conditions were imposed by translational and rotational stiffnesses, and displacements were approximated using Taylor series expansions. Limitations on the first mode of a simply supported beam was simulated with a Taylor series, truncating the series terms up to 3, 5 and 7 terms.

Tao [16] reported a dissertation on deriving analytical solutions to estimate eigen functions for Euler-Bernoulli Beams and Timoshenko Beams with different boundary conditions. Boundary conditions were implemented by considering the two different vertical spring constraints and two different rotational spring constraints. By varying the values of spring constant as zero and infinity, various boundary conditions for beams were demonstrated. 


\subsection{Crack Modeling}

Crack modelling and identification are interesting and challenging topics for researchers from the last three to four decades. Doebling et. al.,[17] presented a comprehensive literature review on identification of structural damage using measured vibrational data. The review is broadly divided into two sections. The first section deals with the damage identification methods and the second section concentrates on the application of damage identification and health monitoring.

In this section, a review on modelling of cracks is presented. The review is broadly divided into two main categories: discrete spring models and local stiffness reduction models. The first category models the crack as a massless rotational spring, while the local stiffness reduction model attempts to model the crack by reducing the local stiffness of the structure at the location of crack. The relevant research in crack modelling is discussed in the following sections.

\subsubsection{Discrete Spring Model}

Khnaijar and Benamar [18] developed a discrete cracked beam model for multiple cracks. The beam bending stiffness was represented as multiple rotational springs representing the crack model and connected by bars. The developed model was validated with reported literature for an EulerBernoulli Beam with simply-supported, clamped-clamped and clamped-free boundary conditions. Spiral springs with a value of zero or infinity were imposed depending on the type of boundary condition. The authors proposed a procedure for crack diagnostics and indicated the necessity for a better description of the crack neighborhood for improved results.

Rizos et al. [19] proposed a non-destructive method to identify the location and depth of cracks in structures by measuring structural modal characteristics. Two harmonic modes of vibration were considered on the left and right side of the crack. The crack was modelled as a rotational spring. The spring constant was calculated using a crack strain energy function and local 
compliance function. The authors concluded that the method lacked accuracy for very small cracks but that the proposed method could be developed into a simple, non-invasive technique and useful tool for preventive maintenance and non-destructive testing of structures. Barad et. al. [20] reported a method for detecting a crack location and size, and validated the results obtained with a mathematical approach. They stated that the eigenvalues of the beam are affected by changes in stiffness and can be used for frequency-based damage detection. A cantilever beam was studied with rotation springs $K_{T}$ representing the damage. The spring constant was estimated using a local compliance function. They concluded that there was a relationship between the crack depth ratio and natural frequencies of the beam.

Yoon et al. [21] demonstrated the influence of two open cracks on the dynamic behavior of double cracked simply-supported beam. An Euler-Bernoulli beam represented by spatial mode functions was considered and the beam was sectioned into 3 parts. The damage-free sections were connected by rotational springs. The stress intensity factor was derived through Castigliano's theorem. Experimentation was carried out to validate the dynamic response and influence of crack depth on stiffness. It was found that the change in natural frequency can be used to estimate the depth of crack. It was also found that the changes in the natural frequencies of a simply-supported beam is inversely proportional to crack depth and the natural frequency decreases rapidly for crack depth ratio higher than 0.5 .

Khiem and Toan [22] demonstrated a modified Rayleigh quotient to calculate natural frequencies of an Euler-Bernoulli beam with multiple cracks by including nonlinear terms. The modified method was further used to detect multiple cracks in the beam. The cracks were modelled using rotational springs, and the spring constant was derived through a local compliance function. The proposed method was validated using experimental and numerical results on a fixed beam. 
Shifrin and Ruotolo [23] proposed a new technique to calculate the eigenvalues of a cracked beam and suggested that the proposed method yields reduced computation time by decreasing the matrix size. In their method, the beam is segmented into $(n+1)$ beams and connected by rotational springs representing the $n$ number of cracks. The flexibility of the rotational spring is calculated using the local compliance function. To demonstrate the proposed technique, a clamped-free Euler-Bernoulli beam boundary condition was considered. They concluded that the procedure opens new possibility in the solving of inverse problem in less time through advanced optimization techniques.

Hu and Liang [24] presented two damage modeling techniques for the identification of cracks in a beam. Euler-Bernoulli beam theory with simply-supported boundary condition was considered. In the first technique, the crack was modelled as a linear and rotational spring. The Castigliano's theorem was used to calculate the stress intensity factor. The second technique was based on a continuum damage concept. The beam was divided into segments with severity of damage ranging through all the segments of the beam. They integrated a massless spring and a continuum crack for the investigation of crack location and the severity of the crack.

Chondros and Dimarogonas [25] investigated the relationship between natural frequency of a cracked beam and the depth of a crack. A cantilever beam welded at a clamped end with a crack on the welded end was studied. The crack was modelled as a torsional spring and assumed to be a transverse surface crack. They established a graphical relation for the local flexibility due to the presence of crack and the depth of crack. They concluded that the established relation can be employed for different beams with different boundary conditions to estimate the depth of crack for known system parameters. It was found as the depth of crack increases, natural frequencies of the cracked system reduce compared to the uncracked system. 
Liang et al. [26] demonstrated a method for quantitative assessment of a beam with a crack and provided an extension to the work for multiple damages. Local flexibility in the vicinity of the crack was developed using a massless rotational spring. The damage location and the magnitude were calculated for simply-supported and cantilever boundary conditions.

Afshari and Inman [27] considered an Euler-Bernoulli Beam with a single crack attached with piezoelectric patch. A new formulation for a cracked beam was demonstrated using the Rayleigh-Ritz method for health monitoring. The crack was modelled as a massless rotational spring to account for the loss of energy. The proposed methodology was demonstrated to be suitable for use with the assumed mode method. In the Rayleigh-Ritz approximation, the mode shapes of the uncracked beam were used. The study was carried out for simply-supported and clamped-clamped boundary conditions for different crack depth rations and normalized locations.

Lee and $\mathrm{Ng}$ [28] used the assumed modes method to estimate the eigenvalues and eigenvectors of a simply-supported Euler-Bernoulli beam with single-sided crack and a pair of double-sided cracks. The crack was modelled as a torsional spring and a linear spring to account for the transverse deflection at the crack. The crack was assumed to divide the beam into two subsections with different sets of admissible functions for each subsection. Their convergence study of the presented model was validated with Galerkin's method and the Finite element method.

Khiem and Lien [29] developed a new method for the dynamic analysis of a beam with an arbitrary number of cracks. The crack was modelled as a rotational spring. The method used the transfer matrix method for the joint crack, for beams with $\mathrm{n}$ internal joint cracks and for a regular beam element. The uniqueness of the method was that it uses a 4 X 4 matrix determinant matrix compared to the referenced paper $(n+2)$ [23]. Numerical studies were carried out on a clamped- 
free Euler-Bernoulli Beam. It was concluded that the present method promises to reduce the computational time for estimation of natural frequency.

Adams et al. [30] evaluated a non-destructive method to study the integrity of structures. A reduced local stiffness and massless spring model was considered for the modelling of the crack. The later model was considered for the study due to its simple applicability. The theoretical and experimental study was carried out on aluminium bar with a saw cut, tapered bar, and camshaft. It was shown the collected vibrational data at a single location could be used as an indicator of magnitude and location of crack.

Saez et.al.[31], presented a simplified method for the evaluation of eigenvalues of an EulerBernoulli beam with a crack. The crack was modelled as an elastic spring. The transverse deflection of an uncracked beam was assumed for the cracked beam with an addition of a polynomial to simplify the process. The beam was modelled as continuous and not dived into twosubsections. The chosen admissible function satisfies the boundary and the kinetic conditions of a specific boundary condition. The Rayleigh method was employed to calculate the natural frequency of an Euler-Bernoulli simply supported beam and validated with results obtained by FEM.

Saez and Navarro [32] presented an analytical method to solve the fundamental frequency of cracked Euler-Bernoulli beams in bending vibrations. The crack was modelled as an elastic rotational spring connecting two segments of the beam. The flexibility influence function method in integral form was employed to estimate the dynamic characteristics of the structure. The discontinuity in the slope due the presence of the crack was proportional to the bending moment. Different boundary conditions were implemented via continuity of displacements, bending moments, and shear forces. The results were validated with numerical simulation results for simply 
supported cracked beam, cantilever cracked beam, fixed-pinned cracked beam and fixed-fixed cracked beam.

\subsubsection{Local Stiffness Reduction}

Ostachowicz and Kalwczak [33] presented an analytical study of a cracked cantilever beam. Two types of cracks are assumed. The single-sided crack is a result of a fluctuating load, and the doublesided load is a result of a cyclic load. The crack is modelled using a stress intensity factor and it is assumed that there is decrease in elastic deformation of energy in the plane stress of the beam. Different types of loading were discussed, and numerical examples were presented.

Chondros et. al.,[34] developed a continuous cracked bar vibration model for an EulerBernoulli cantilever beam with a crack. The crack was assumed to be an edge crack and was modelled as continuous flexibility. Using stress intensity factor and Castigliano's theorem, the differential equation for cracked beam was developed. It was suggested that the continuous cracked beam theory holds applications for cracked structures.

Yang et.at.,[35] developed a numerical model to evaluate the dynamic characteristics of a cracked beam. The crack is assumed to be an open $\operatorname{crack}(\mathrm{s})$. The crack is modelled using the strain energy. To evaluate the natural frequencies, a four-term Galerkin's method was used. A simplysupported and a fixed-fixed beam were considered for the study of the proposed model. It was concluded that the change in the natural frequency of the beam due to the presence of crack depends on the crack size and crack location. If the crack is located near the boundaries, the boundary conditions tend to modify. However, if a crack coincides with a node of one the vibrating modes, the natural frequency at that mode remains unchanged.

Qian et Al [36] proposed a simple method to determine the crack location based on the relationship between the crack and the eigencouple. The crack was assumed to be an edge-crack 
on a cantilever beam. Using the stress intensity factor and the finite element method, the stiffness matrix of the cracked beam was developed. The proposed method is suggested for complex structures with known stress intensity factors.

Kam and Lee [37] presented an alternative approach for a non-destructive method to identify the size of the crack for a given location of the crack in the structure. The crack is modelled as in [36]. The strain energy of the uncracked and cracked beam was utilized to determine the crack size iteratively. The effect of the presence of the crack on the structural strain energy was obtained based on fracture mechanics. The study was carried out for different beams and frame structures. The proposed method was verified with FEM.

Sinha et. al.[38] proposed a simplified crack modelling approach using an FEM approach for estimation of crack depth and location. The crack was modelled using the concept of change in stress and strain in the vicinity of the crack section. The study used Euler-Bernoulli beam elements with minor modifications to the local flexibility in the vicinity of cracks. The model was simplified compared to older models by involving the crack location and depth directly by modelling the crack with triangular reduction in stiffness. The study was carried out on an aluminium cantilever beam, an aluminium free-free beam, and a steel free-free beam. The validation was carried out with experimental data and other crack models.

Lu et.al.,[39] presented a two-step approach to identify cracks based on modeshapes of structures and sensitivity analysis. The crack was modelled as a local stiffness reduction, similar to the crack model used in [38]. In the first step, the change in the modeshape of the uncracked and cracked beam was studied to locate the crack. The second step involved model updating based on response sensitivity to accurately locate the crack and the depth of crack. The proposed method was validated numerically on a cracked steel cantilever beam and a simply supported beam. 
Kam and Lee [40] presented a general procedure to locate a single crack in structures using modal data. The crack was modelled using a reduced stiffness model. It was assumed that the presence of the crack only effects the stress field of the regions adjacent to the crack. Kam and Lee presented an approach to estimate the crack size using static deflection analyses. To evaluate the presented approach, a cantilever Euler-Bernoulli beam was used to obtain the modal data experimentally or using FEM. The presented approach with slight modifications could be further extended to multiple crack structures.

\subsection{Summary}

In this Chapter 2, a literature review carried out and subdivided into three sections. The first section starts with literature on the various trial functions used in modeling beams using the assumed modes method. It is observed that over the years different trial functions were used to obtain the dynamic characteristics of beams or plates. The choice of set of functions often lead to numerical instability and/or slower convergence. In addition, the change in boundary conditions usually requires a change in the selected trail functions. In this thesis, a set of Alternative Admissible Functions $(A A F)[2]$ are selected to calculate the dynamic characteristics of an unconstrained beam. The $A A F$ overcomes the issues faced by the other trial functions used over the course of time.

The second section focuses on the literature related to penalty functions and existing values of penalties used in the analysis. It is noted that to the best of the author's knowledge, a study on selection and estimation of different boundary conditions using $A A F$ has not been considered. This provides a scope to derive a relationship for the calculation of the boundary conditions based on the problem statement. 
Structure condition monitoring is an important element of any structure's life cycle. In condition monitoring, qualitative and quantitative examination of structural deterioration is critical. Cracks are one type of damage. As a result, if there are any future condition monitoring issues, modelling cracks is the first step. In the third section, the literature on modelling beams with cracks and applications of this modelling is discussed. The discrete spring model and the local stiffness reduction model are the two predominant approaches that summarise the different crack modelling approaches employed in the present literature review.

The discrete spring model is a popular model because of its simplicity. A rotational/torsional spring is used to represent the crack. The crack strain energy function and the local compliance function are used to determine the spring constant. From the location of the crack, the beams were generally separated into two segments. For the left and right sides of the beam to be discretized into two parts, two separate sets of trial functions are necessary. When the beam was not separated into segments, the trial functions employed to represent the entire beam with crack were chosen to satisfy the beam boundary conditions, resulting in a function change with each change in boundary condition.

The local stiffness reduction method models the crack as a local reduction in stiffness. Using stress intensity factor, Castigliano's theorem and a few other theorems, the crack stiffness is quantified. Since the method focuses on the local stiffness reduction, FEM methods are often used to study dynamic characteristics of the beam. Both methods discussed find application in various field of dynamic study of structures.

Based on this literature review and to the best of the author's knowledge, modelling of a crack as a penalty function using massless rotational springs along with $A F F$ with boundary penalties to estimate the natural frequency of a cracked beam has not been considered so far. 


\section{Mathematical Model using Alternative Admissible Functions}

\subsection{Introduction}

Structural dynamics is an active research area due to its widespread importance in many engineering applications. Many attempts have been made by researchers in the past to develop methods to compute system dynamic characteristics such as eigenvalues (natural frequencies), eigenvectors (modeshapes), damping characteristics, structural response, and frequency response functions ( $F R F$ 's). Analytical approaches often make uses of true eigenfunctions, which can often involve hyperbolic functions whose behaviors are numerically unstable. In this chapter, the use of Alternative Admissible Functions $(A A F)$ for the calculation of system natural frequencies and modeshapes is introduced and demonstrated. These $A A F$ are numerically stable and do not display the ill-conditioning that can occur when the analytical eigenfunctions are used. Applying the Rayleigh-Ritz minimization to a conservative beam system, unconstrained mass and stiffness matrices are obtained and reported. Three commonly used boundary conditions viz., ClampedFree $(C F)$, Simply-Supported (SS) and Clamped-Clamped $(C C)$ are imposed on the free-free beam by virtual stiffnesses, using penalty terms composed of linear $\left(k_{0}\right)$ and rotational $\left(k_{r}\right)$ springs.

To investigate the behaviour of the proposed approach, different geometrical dimensions, especially the length and thickness of a beam, as well as the beam material are considered. Extracted eigenvalues and eigenvectors are compared with closed-form Euler-Bernoulli beam values as a benchmark. The work is presented in a hierarchical manner detailing about the use of $A A F$ for structural dynamics, mathematical formulation for estimation of $k_{0}$ and $k_{r 0}$ matrices, and convergence of value of penalty functions. 


\subsection{Alternative Admissible Functions}

The proposed alternative admissible functions $(A A F)$ consist of polynomials and trigonometric functions. The selected set of alternative admissible functions have been demonstrated to be easy to compute and numerically well behaved [3] for applications to plates. The number of terms, $N$, to use, and the choice of penalty terms play a crucial role in the $A A F$ method. The increase in the number of terms increases the computational time and the value of the penalty terms needs to be selected based on the problem statement. As a general rule of thumb for assumed mode approaches, Meirovitch [1] suggested that $N$ should be twice the number of frequencies of interest. Prior research has indicated that large values for the penalty terms should generally be assumed [3].

The following section of the thesis focuses on the study of Alternative Admissible Functions method on beams, formulation of the equation of motion for the unconstrained beam and the use of penalty functions with different boundary conditions. The effectiveness of method is studied using geometrical variations for application to Euler-Bernoulli beams.

\subsection{Derivation of Equation of Motion}

This section elaborates the discussion on defining the system, formulation of the assumed-modes method and normalized admissible functions for the unconstrained system. A Euler- Bernoulli beam, as shown in Figure 3-1, is considered throughout the present study.
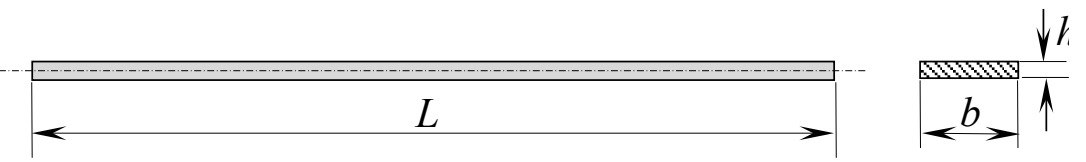

Figure 3-1: Euler-Bernoulli Beam

As shown in Figure 3-1, $L$ is the length of the beam, $b$ is the breadth/width and $h$ are the thickness of the beam. All dimensions are assumed to be in meters. The material properties of the beam are given as $E$ for young's modulus in $\mathrm{N} / \mathrm{m}^{2}$ and $\rho$ is the density per unit length of the beam in $\mathrm{kg} / \mathrm{m}$. 


\subsubsection{Defining System}

The assumed shape method is a technique used to model a continuous system as a discrete one, thereby simplifying the solution. The assumed shape method is used to determine the equation of motion of a system, from which an eigenvalue solution may be determined. The assumed-modes method for the discretization of distributed-parameter systems is closely related to the RayleighRitz method $(R R M)$, but the assumed-modes method is easier to implement compared to RRM [1]. For the assumed modes method, the transverse displacement $w(x, t)$ of the oscillations of an EulerBernoulli beam is modelled using an assumed-modes approach and the form is given by

$$
w(x, t)=W(x) \cos (\omega t)
$$

where $x$ is the position along the beam, $t$ is time and $\omega$ is the frequency of vibration. The spatial function, $W(x)$ is assumed to be a sum of assumed-modes and to have the form given by

$$
W(x)=\sum_{i=1}^{N} c_{i} \emptyset_{i}(x)
$$

where $\phi_{i}(\mathrm{x})$ is the set of assumed modes, $c_{i}$ are constants and $N$ are the number of Alterative Admissible Functions terms, as in $\emptyset_{i}(i=1,2, \ldots, N)$. In the present work, the chosen set of alternative admissible functions are as indicated by Monterrubio and Ilanko [2] and are given by

$$
\begin{aligned}
& \phi_{i}(x)=1 \quad \text { for } i=1 \\
& \phi_{i}(x)=\left(\frac{x}{L}\right) \text { for } i=2 \\
& \phi_{i}(x)=\left(\frac{x}{L}\right)^{2} \text { for } i=3 \\
& \phi_{i}(x)=\cos \frac{(i-3) \pi x}{L} \text { for } i=4,5,6, \ldots . N
\end{aligned}
$$


The choice of the set of admissible functions presented in Eq. (3.3) is such that the functions can best represent the eigenvector of a structure in an unconstrained condition [2]. In the traditional application of the assumed modes method, the comparison functions are often chosen as eigenfunctions of the system and thus satisfy the boundary conditions. The advantage of this selected set of admissible functions is that it can be used with different boundary conditions without altering the choice of assumed mode functions, as will be elaborated below. The first five alternative admissible functions are shown in Figure 3-2.

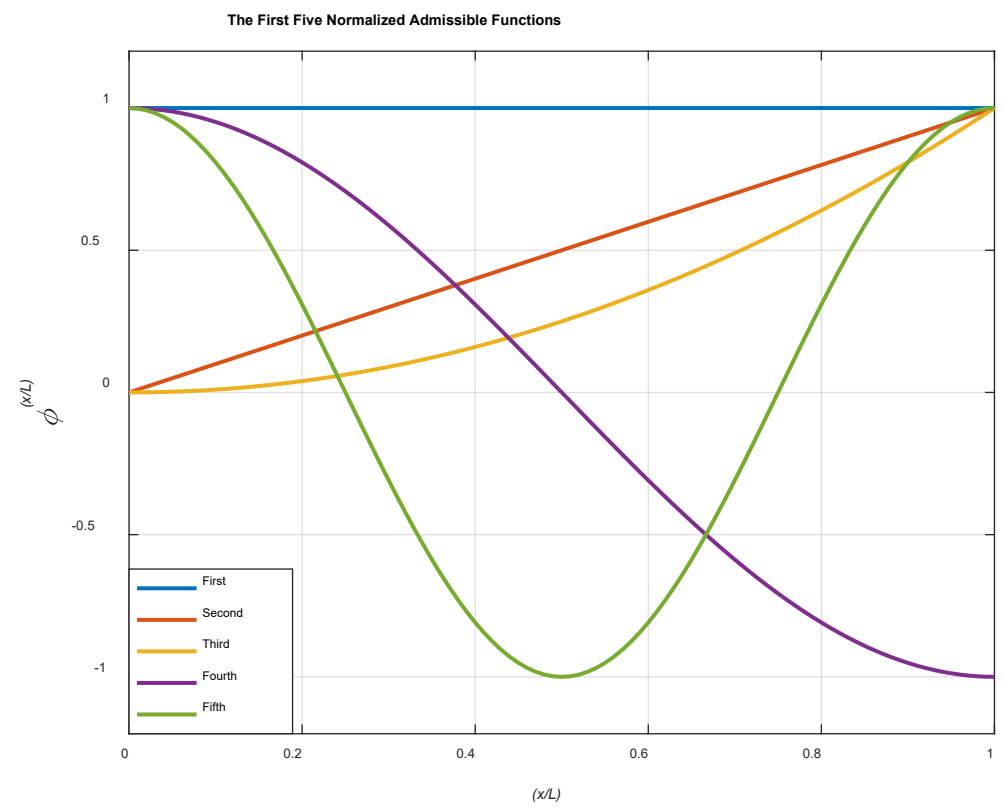

Figure 3-2: First Five Normalized Alternative Admissible Functions

Knowing the system parameters such as the mechanical properties (Young's modulus $E$ and density per length of the beam, $\rho$ ) and geometrical parameters (length $L$, width $b$ and height $h$ ), then the maximum kinetic energy $\left(T_{\max }\right)$ and maximum potential energy $\left(V_{\max }\right)$ of the unconstrained system can be written as

$$
T_{\text {max }}=\frac{\rho \omega^{2}}{2} \int_{x=0}^{x=L} W^{2}(x) d x
$$

and 


$$
V_{\max }=\frac{E I}{2} \int_{x=0}^{x=L}\left(\frac{\partial^{2} W}{\partial x^{2}}\right)^{2} d x
$$

where, $I$ is the second moment of area of the cross section, $T_{\max }$ and $V_{\max }$ are defined in terms of the admissible functions. For conservative system, ignoring the damping, it takes the form $V_{\max }-$ $T_{\text {max }}=0$. Applying the Rayleigh-Ritz minimization to the conservative system leads to the derivation of mass and stiffness matrices using $\frac{\partial V_{\max }}{\partial c_{i}}-\omega^{2} \frac{\partial \Gamma_{\max }}{\partial c_{i}}=0 \quad i=1,2 \ldots \ldots N$, where $\Gamma_{\max }=\frac{T_{\max }}{\omega^{2}}$ is the normalized maximum kinetic energy function to be minimized. On solving $\frac{\partial V_{\max }}{\partial c_{i}}-\omega^{2} \frac{\partial \Gamma_{\max }}{\partial c_{i}}=0 \quad i=1,2 \ldots . . N$ results in a set of $N$ algebraic equations whose equivalent matrix entries (i.e. mass and stiffness matrices) are obtained in the forms respectively given by

$$
m_{i j}=m_{j i}=\rho \int_{0}^{L} \phi_{i}(x) \phi_{j}(x) d x
$$

and,

$$
k_{i j}=k_{j i}=E I \int_{0}^{L} \phi_{i}{ }^{\prime \prime}(x) \phi_{j}{ }^{\prime \prime}(x) d x
$$

where $m_{i j}$ denote the entries of the mass matrix $M$ and $k_{i j}$ denote the entries in the stiffness matrix, $K$. Furthermore, equation of motion for the unconstrained system can be written in generalized matrix form as,

$$
\left[K-\omega^{2} M\right] \mathrm{C}=0
$$

The vector $C$ is the vector of coefficients from Eq. (3.2) defined as,

$$
C=\left\{\begin{array}{c}
c_{1} \\
c_{2} \\
\cdot \\
\cdot \\
c_{N}
\end{array}\right\}
$$


For a non-trivial solution of Eq. (3.8), the determinant of the coefficients of $C$ must be equal to zero, hence

$$
\left|K-\omega^{2} M\right|=0
$$

Eq.(3.8) and Eq.(3.10) can be used to calculate the natural frequencies and the modeshapes of a beam with Free-Free $(F F)$ boundary conditions (unconstrained beam). Other boundary conditions such as Clamped-Free $(C F)$, Simply-Supported $(S S)$ and Clamped-Clamped $(C C)$ can be imposed by using virtual springs (constraints) appropriately at the beam ends. This is accomplished by using penalty functions, as explained in the section 3.5. The steps for obtaining the unconstrained mass and stiffness matrices of a free-free beam are detailed in the next section.

\subsection{Unconstrained Mass and Stiffness Matrices}

The unconstrained mass and stiffness matrices of a free-free beam are calculated from Eq. (3.6) and Eq.(3.7), respectively. Referring to Eq.(3.2), and considering $N=5$, the alternative admissible functions, their first and second derivatives are tabulated in Table 3-1.

Table 3-1: Admissible Functions and 1st and 2nd Derivative

\begin{tabular}{|c|c|c|c|}
\hline $\boldsymbol{N}$ & $\emptyset_{\mathbf{i}}(\boldsymbol{x})$ & $\emptyset_{\mathbf{i}}^{\prime}(\boldsymbol{x})$ & $\emptyset_{\mathbf{i}}^{\prime \prime}(\boldsymbol{x})$ \\
\hline 1 & 1 & 0 & 0 \\
\hline 2 & $\left(\frac{x}{L}\right)$ & $\left(\frac{1}{L}\right)$ & $\left(\frac{2}{L^{2}}\right)$ \\
\hline 3 & $\left(\frac{x}{L}\right)^{2}$ & $\left(\frac{2 x}{L^{2}}\right)$ & $-\left(\frac{\pi}{L}\right)^{2} \cos \frac{\pi x}{L}$ \\
\hline 4 & $\cos \frac{\pi x}{L}$ & $-\frac{\pi}{L} \sin \frac{\pi x}{L}$ & $-\left(\frac{2 \pi}{L}\right)^{2} \cos \frac{2 \pi x}{L}$ \\
\hline 5 & $\cos \frac{2 \pi x}{L}$ & $-\frac{2 \pi}{L} \sin \frac{2 \pi x}{L}$ & \multicolumn{2}{|c|}{} \\
\hline
\end{tabular}

Using Eq.(3.2) and admissible functions as listed in Table 3-1, symmetric unconstrained mass and stiffness matrices are obtained for the $N=5$ case as follows, 


$$
M=\rho L\left[\begin{array}{ccccc}
1 & 1 / 2 & 1 / 3 & 0 & 0 \\
1 / 2 & 1 / 3 & 1 / 4 & -2 / \pi^{2} & 0 \\
1 / 3 & 1 / 4 & 1 / 5 & -2 / \pi^{2} & 1 / 2 \pi^{2} \\
0 & -2 / \pi^{2} & -2 / \pi^{2} & 1 / 2 & 0 \\
0 & 0 & 1 / 2 \pi^{2} & 0 & 1 / 2
\end{array}\right]
$$

and

$$
K=\frac{E I}{L^{3}}\left[\begin{array}{ccccc}
0 & 0 & 0 & 0 & 0 \\
0 & 0 & 0 & 0 & 0 \\
0 & 0 & 4 & 0 & 0 \\
0 & 0 & 0 & \pi^{4} / 2 & 0 \\
0 & 0 & 0 & 0 & 8 \pi^{4}
\end{array}\right]
$$

With the use of alternative admissible functions, changes in boundary conditions do not alter the mass and stiffness matrices given in Eq.(3.11) and Eq. (3.12). This stands in contrast to the traditional implementation of assumed modes approaches where the form of the assumed mode is typically changed with a change in the boundary conditions, hence changing the system mass and stiffness matrices. With the $A A F$ approach, changes in boundary conditions are implemented via penalty matrices, the implementation of which is explained below.

\subsection{Penalty Functions}

With the $A A F$ approach, the selected set of assumed modes do not satisfy the boundary conditions of the beam and therefore, penalty terms are introduced to satisfy the different boundary conditions. The boundary conditions are satisfied by introducing virtual stiffnesses represented via linear springs $\left(k_{0}\right)$ and rotational springs $\left(k_{r 0}\right)$ on the boundary. The system potential energy is altered due to the addition of the penalty terms. The potential energy terms associated with these virtual springs will modify the system stiffness matrix and will thus be introduced into the Rayleigh quotient. Most generally, the penalty function associated with the potential energy of virtual springs on the boundary is given by 


$$
V_{p}=\left.\frac{k_{0}}{2} W^{2}\right|_{x=0}+\left.\frac{k_{0}}{2} W^{2}\right|_{x=L}+\left.\frac{k_{r 0}}{2} L^{2}\left(\frac{\partial W}{\partial x}\right)^{2}\right|_{x=0}+\left.\frac{k_{r 0}}{2} L^{2}\left(\frac{\partial W}{\partial x}\right)^{2}\right|_{x=L}
$$

The present work uses the alternative admissible functions as given in Eq. (3.3), which combines polynomials up to the second degree, and a cosine Fourier series.

Incorporating the assumed modes of Eq. (3.3) into (3.13) and following the procedure outlined previously results in the penalty stiffness matrices of the problem.

The symmetric penalty stiffness matrix is given by,

$$
k_{P, i j}=k_{0} \phi_{i}(0) \phi_{j}(0)+k_{0} \phi_{i}(L) \phi_{j}(L)+k_{r 0} L^{2} \phi_{i}^{\prime}(0) \phi_{j}^{\prime}(0)+k_{r 0} L^{2} \phi_{i}^{\prime}(L) \phi_{j}^{\prime}(L)
$$

The first two terms in Eq.(3.14) indicate the virtual linear springs $\left(k_{0}\right)$ and last two terms indicate the virtual rotational springs $\left(k_{r 0}\right)$ at the boundaries of the beam. The virtual linear springs and the rotational springs restrict linear and rotational movements, respectively. Depending on the boundary conditions of the problem, a combination of appropriate virtual spring elements can be added to the stiffness matrix. A few commonly used boundary conditions and their corresponding symmetric penalty stiffness matrix are shown in the following subsections.

\subsubsection{Clamped-Free (CF) Boundary condition}

For a Clamped-Free $(C F)$ boundary condition, one virtual linear spring $\left(k_{0}\right)$ and one virtual rotational spring $\left(k_{r}\right)$ are imposed at one end of the beam and other end is left free, as shown in Figure 3-3.

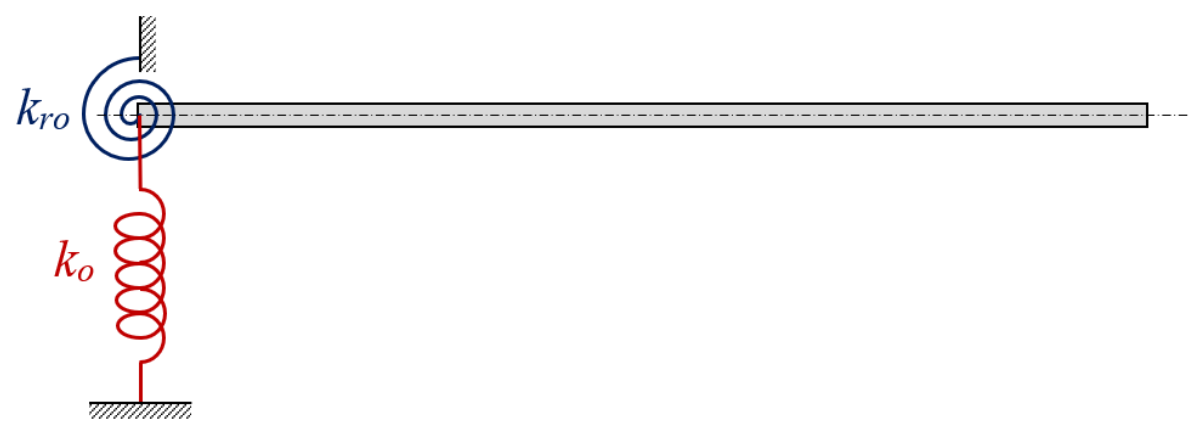

Figure 3-3: Beam with Clamped-Free $(C F)$ Boundary Condition 
The energy of the virtual linear spring is represented by $k_{0} \phi_{i}(0) \phi_{j}(0)$ and constrains the vertical movement of the beam. Meanwhile, $k_{r 0} L^{2} \phi_{i}^{\prime}(L) \phi_{j}^{\prime}(L)$ represents the rotational spring energy and constrains the rotation of the beam. The penalty stiffness matrix for clamped-free boundary condition is given then by as

$$
k_{P, C F i j}=k_{0} \phi_{i}(0) \phi_{j}(0)+k_{r 0} L^{2} \phi_{i}^{\prime}(0) \phi_{j}^{\prime}(0)
$$

The sum of the system free-free beam stiffness matrix (3.7) and the clamped-free boundary condition penalty stiffness matrix (3.15) gives the total stiffness matrix for clamped-free beam $\left(K_{T}\right)$.

$$
K_{T}=K+K_{P, C F}
$$

It can be observed that $K_{T}$ houses all the information about the unconstrained beam stiffness and the clamped-free boundary conditions. With the change in the boundary condition only the penalty stiffness needs to be altered.

\subsubsection{Simply-Supported (SS) Boundary condition}

A Simply-Supported (SS) beam boundary condition is imposed using two virtual linear springs $\left(k_{0}\right)$ at the ends of the beam to inhibit any vertical movement. Virtual rotational spring $\left(k_{r 0}\right)$ are not used, thereby allowing free rotation at both ends which models the simply supported boundary condition. Figure 3-4 represents a simply-supported beam with two linear virtual springs.

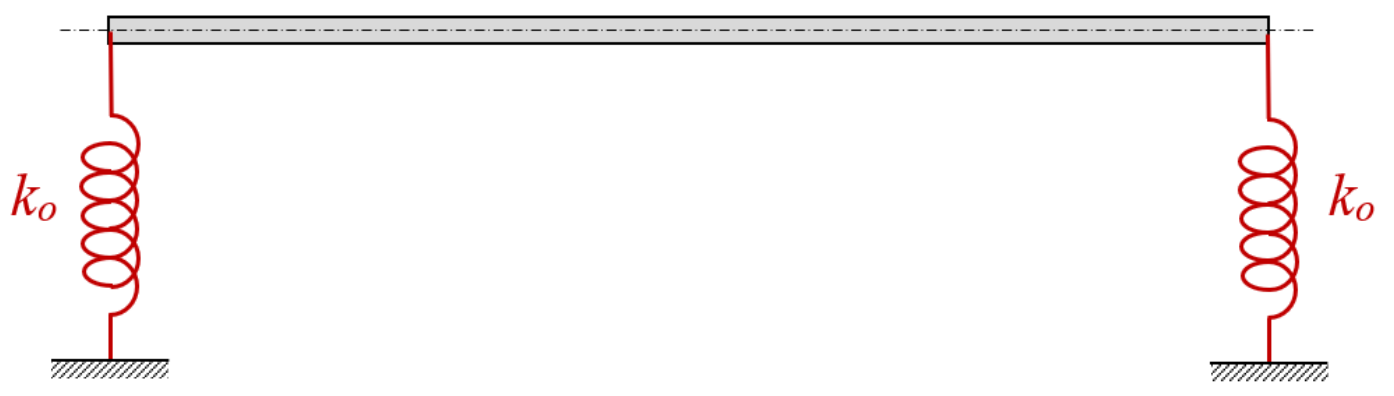

Figure 3-4: Beam with Simply-Supported (SS) Boundary Condition 
The energy of the left-hand virtual linear spring is represented by $k_{0} \phi_{i}(0) \phi_{j}(0)$ and constrains the vertical movement of the beam at the left boundary condition. In Eq.(3.16), $k_{0} \phi_{i}(0) \phi_{j}(0)$ and $k_{0} \phi_{i}(L) \phi_{j}(L)$, impose the virtual linear springs at $x=0$ and $x=L$ respectively. The penalty stiffness matrix for the simply-supported boundary conditions is given by Eq.(3.16) as

$$
k_{P, S S i j}=k_{0} \phi_{i}(0) \phi_{j}(0)+k_{0} \phi_{i}(L) \phi_{j}(L)
$$

The sum of the free-free beam stiffness matrix and the simply-supported boundary condition penalty stiffness matrix gives the total stiffness matrix simply-supported beam $\left(K_{T}\right)$.

\subsubsection{Clamped-Clamped $(C C)$ Boundary condition}

For a Clamped-Clamped $(C C)$ boundary condition, a combination of virtual linear spring and virtual rotational spring are imposed on both ends of the beam. Figure 3-5 shows a clampedclamped beam boundary condition.

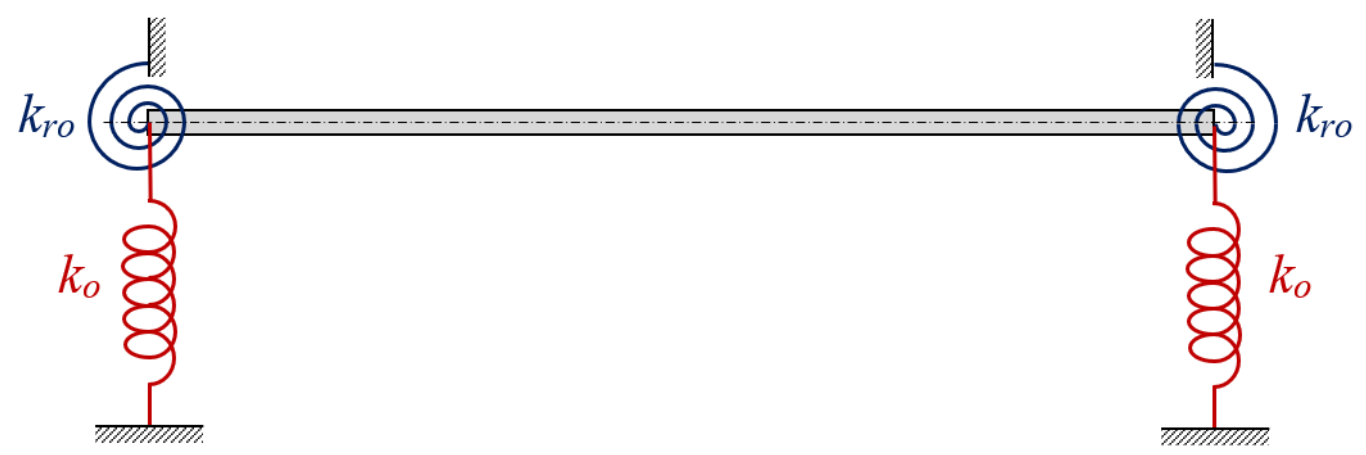

Figure 3-5: Beam with Clamped-Clamped (CC) Boundary Condition

Eq.(3.17) represents a combination of linear and rotational springs. The $k_{0} \phi_{i}(0) \phi_{j}(0)$ and $k_{r 0} L^{2} \phi_{i}^{\prime}(0) \phi_{j}^{\prime}(0)$, represent the virtual linear springs and virtual rotational spring at the $x=0$ end, respectively. The $k_{0} \phi_{i}(L) \phi_{j}(L)$ and $k_{r 0} L^{2} \phi_{i}^{\prime}(L) \phi_{j}^{\prime}(L)$, represent the virtual linear springs and virtual rotational spring at the $x=L$ end, respectively. Thus, the entries of the total penalty (boundary condition) stiffness matrix are given by 


$$
\begin{gathered}
k_{P, C C i j}=k_{0} \phi_{i}(0) \phi_{j}(0)+k_{0} \phi_{i}(L) \phi_{j}(L)+k_{r 0} L^{2} \phi_{i}^{\prime}(0) \phi_{j}^{\prime}(0) \\
+k_{r 0} L^{2} \phi_{i}^{\prime}(L) \phi_{j}^{\prime}(L)
\end{gathered}
$$

The obtained penalty terms for different boundary conditions are then summed with the unconstrained stiffness matrix of the free-free beam to obtain the total system stiffness matrix $\left(K_{T}\right)$.

\subsection{Penalty Stiffness Matrix for Different Boundary Conditions}

To develop the penalty stiffness matrix for different boundary conditions, the use of virtual springs is utilized. Using the potential energy and proposed alternative admissible functions, the matrices for Clamped-Free $(C F)$, Simply-Supported (SS) and, Clamped-Clamped $(C C)$ are computed in this section.

\subsubsection{Penalty Stiffness Matrix for Clamped-Free $(\mathrm{CF})$ Condition}

The penalty stiffness matrix for clamped-free boundary conditions is computed using Eq.(3.15) and admissible functions listed in Table 3-1. The first and second part of Eq.(3.15) are tabulated in Table 3-2 and Table 3-3 and give the symmetric penalty stiffness matrix for Clamped-Free $(C F)$ boundary condition. 
Table 3-2: Clamped-Free (CF) First Part of Eq.(3.15)

\begin{tabular}{|c|c|c|c|c|c|c|c|}
\hline \multicolumn{8}{|c|}{$\boldsymbol{k}_{\mathbf{0}} \emptyset_{i}(\mathbf{0}) \emptyset_{j}(\mathbf{0})$} \\
\hline & & & 1 & 2 & 3 & 4 & 5 \\
\hline & & & 1 & $\left(\frac{x}{L}\right)$ & $\left(\frac{x}{L}\right)^{2}$ & $\cos \frac{\pi x}{L}$ & $\cos \frac{2 \pi x}{L}$ \\
\hline & & $\emptyset_{i j}(0)$ & 1 & 0 & 0 & 1 & 1 \\
\hline 1 & 1 & 1 & $k_{0}$ & 0 & 0 & $k_{0}$ & $k_{0}$ \\
\hline 2 & $\left(\frac{x}{L}\right)$ & 0 & 0 & 0 & 0 & 0 & 0 \\
\hline 3 & $\left(\frac{x}{L}\right)^{2}$ & 0 & 0 & 0 & 0 & 0 & 0 \\
\hline 4 & $\cos \frac{\pi x}{L}$ & 1 & $k_{0}$ & 0 & 0 & $k_{0}$ & $\boldsymbol{k}_{0}$ \\
\hline 5 & $\cos \frac{2 \pi x}{L}$ & 1 & $k_{0}$ & 0 & 0 & $k_{0}$ & $k_{0}$ \\
\hline
\end{tabular}

Table 3-3: Clamped- Free (CF) Second Part of Eq.(3.15)

\begin{tabular}{|c|c|c|c|c|c|c|c|}
\hline \multicolumn{8}{|c|}{$k_{r 0} L^{2} \emptyset_{i}^{\prime}(0) \emptyset_{j}^{\prime}(0)$} \\
\hline & & & 1 & 2 & 3 & 4 & 5 \\
\hline & & & 0 & $\left(\frac{1}{L}\right)$ & $\left(\frac{2 x}{L^{2}}\right)$ & $-\frac{\pi}{L} \sin \frac{\pi x}{L}$ & $-\frac{2 \pi}{L} \sin \frac{2 \pi x}{L}$ \\
\hline & & $\emptyset_{i j}^{\prime}(0)$ & 0 & $\left(\frac{1}{L}\right)$ & 0 & 0 & 0 \\
\hline 1 & 0 & 0 & 0 & 0 & 0 & 0 & 0 \\
\hline 2 & $\left(\frac{1}{L}\right)$ & $\left(\frac{1}{L}\right)$ & 0 & $k_{r 0}$ & 0 & 0 & 0 \\
\hline 3 & $\left(\frac{2 x}{L^{2}}\right)$ & 0 & 0 & 0 & 0 & 0 & 0 \\
\hline 4 & $-\frac{\pi}{L} \sin \frac{\pi x}{L}$ & 0 & 0 & 0 & 0 & 0 & 0 \\
\hline 5 & $-\frac{2 \pi}{L} \sin \frac{2 \pi x}{L}$ & 0 & 0 & 0 & 0 & 0 & 0 \\
\hline
\end{tabular}


The tables are subject to matrix addition and thus the penalty matrix for the Clamped-Free (CF) boundary condition is obtained. Eq.(3.18) gives the penalty term matrix for the clamped-free boundary condition as

$$
K_{P, C F}=\left[\begin{array}{ccccc}
k_{0} & 0 & 0 & k_{0} & k_{0} \\
0 & k_{r 0} & 0 & 0 & 0 \\
0 & 0 & 0 & 0 & 0 \\
k_{0} & 0 & 0 & k_{0} & k_{0} \\
k_{0} & 0 & 0 & k_{0} & k_{0}
\end{array}\right]
$$

The $k_{0}$ and $k_{r 0}$ represent the virtual linear and rotational spring in the symmetric penalty stiffness matrix for Clamped-Free $(C F)$ boundary condition shown in Eq.(3.18).

\subsubsection{Penalty Stiffness Matrix for Simply-Supported (SS) Condition}

Similarly using Eq.(3.16) with the admissible functions listed in Table 3-1 the symmetric penalty stiffness matrix for Simply-Supported (SS) conditions are determined. The first and second part of Eq.(3.16) are shown in Table 3-4 and Table 3-5 and give the symmetric penalty stiffness matrix for Simply-Supported (SS) boundary condition.

Table 3-4: Simply-Supported (SS) First Part of Eq.(3.16)

\begin{tabular}{|c|c|c|c|c|c|c|c|}
\hline \multicolumn{2}{|c|}{} & & 1 & 2 & 3 & 4 & 5 \\
\cline { 3 - 8 } & & 1 & $\left(\frac{\mathrm{X}}{\mathrm{L}}\right)$ & $\left(\frac{\mathrm{X}}{\mathrm{L}}\right)^{2}$ & $\cos \frac{\pi \mathrm{x}}{\mathrm{L}}$ & $\cos \frac{2 \pi \mathrm{x}}{\mathrm{L}}$ \\
\cline { 3 - 8 }$\emptyset_{\mathrm{ij}}(0)$ & 1 & 0 & 0 & 1 & 1 \\
\hline 1 & 1 & 1 & $\boldsymbol{k}_{\mathbf{0}}$ & 0 & 0 & $\boldsymbol{k}_{\mathbf{0}}$ & $\boldsymbol{k}_{\mathbf{0}}$ \\
\hline 2 & $\left(\frac{\mathrm{X}}{\mathrm{L}}\right)$ & 0 & 0 & 0 & 0 & 0 & 0 \\
\hline 3 & $\left(\frac{\mathrm{x}}{\mathrm{L}}\right)^{2}$ & 0 & 0 & 0 & 0 & 0 & 0 \\
\hline 4 & $\cos \frac{\pi \mathrm{x}}{\mathrm{L}}$ & 1 & $\boldsymbol{k}_{\mathbf{0}}$ & 0 & 0 & $\boldsymbol{k}_{\mathbf{0}}$ & $\boldsymbol{k}_{\mathbf{0}}$ \\
\hline 5 & $\cos \frac{2 \pi \mathrm{x}}{\mathrm{L}}$ & 1 & $\boldsymbol{k}_{\mathbf{0}}$ & 0 & 0 & $\boldsymbol{k}_{\mathbf{0}}$ & $\boldsymbol{k}_{\mathbf{0}}$ \\
\hline
\end{tabular}


Table 3-5: Simply-Supported (SS) Second Part of Eq.(3.16)

\begin{tabular}{|c|c|c|c|c|c|c|c|}
\hline \multicolumn{8}{|c|}{$\boldsymbol{k}_{\mathbf{0}} \emptyset_{i}(\boldsymbol{L}) \emptyset_{j}(\boldsymbol{L})$} \\
\hline & & & 1 & 2 & 3 & 4 & 5 \\
\hline & & & 1 & $\left(\frac{x}{-1}\right)$ & $(x)^{2}$ & $\pi x$ & $2 \pi x$ \\
\hline & & $\emptyset_{i j}(L)$ & 1 & 1 & 1 & -1 & 1 \\
\hline 1 & 1 & 1 & $\boldsymbol{k}_{0}$ & $k_{0}$ & $k_{0}$ & $-\boldsymbol{k}_{0}$ & $k_{0}$ \\
\hline 2 & $\left(\frac{x}{L}\right)$ & 1 & $k_{0}$ & $k_{0}$ & $\boldsymbol{k}_{0}$ & $-\boldsymbol{k}_{0}$ & $k_{0}$ \\
\hline 3 & $\left(\frac{x}{I}\right)^{2}$ & 1 & $k_{0}$ & $k_{0}$ & $\boldsymbol{k}_{0}$ & $-\boldsymbol{k}_{0}$ & $k_{0}$ \\
\hline 4 & $\cos \frac{\pi x}{L}$ & -1 & $-\boldsymbol{k}_{0}$ & $-\boldsymbol{k}_{0}$ & $-\boldsymbol{k}_{0}$ & $k_{0}$ & $-\boldsymbol{k}_{0}$ \\
\hline 5 & $\cos \frac{2 \pi x}{L}$ & 1 & $k_{0}$ & $\boldsymbol{k}_{0}$ & $\boldsymbol{k}_{0}$ & $-\boldsymbol{k}_{0}$ & $\boldsymbol{k}_{0}$ \\
\hline
\end{tabular}

The tables are subject to matrix addition and hence the penalty matrix for the Simply-Supported (SS) boundary condition is obtained. The penalty term matrix for simply-supported boundary condition is given by Eq.(3.19) as

$$
K_{P, S S}=\left[\begin{array}{ccccc}
2 k_{0} & k_{0} & k_{0} & 0 & 2 k_{0} \\
k_{0} & k_{0} & k_{0} & -k_{0} & k_{0} \\
k_{0} & k_{0} & k_{0} & -k_{0} & k_{0} \\
0 & -k_{0} & -k_{0} & 2 k_{0} & 0 \\
2 k_{0} & k_{0} & k_{0} & 0 & 2 k_{0}
\end{array}\right]
$$

It can be observed that the penalty matrix for the simply-supported beam only has virtual linear springs, as the beam is only constrained to move vertically and can rotate freely at the boundary. 


\subsubsection{Penalty Stiffness Matrix for Clamped-Clamped (CC) Condition}

To obtain symmetric penalty stiffness matrix for the Clamped-Clamped (CC) boundary condition, Eq.(3.17) and Table 3-1 are used. The penalty matrix for $C C$ conditions has four components and follows the same procedure as explained above for $C F$ and $S S$ conditions. The symmetric penalty stiffness matrix for Clamped-Clamped $(\boldsymbol{C C})$ conditions are determined via the tabulated values shown in Table 3-6, Table 3-7, Table 3-8, and Table 3-9.

Table 3-6: Clamped-Clamped (CC) First Part of Eq.(3.17)

\begin{tabular}{|c|c|c|c|c|c|c|c|}
\hline \multicolumn{7}{|c|}{$\mathbf{k}_{\mathbf{0}} \emptyset_{\mathbf{i}}(\mathbf{0}) \emptyset_{\mathrm{j}}(\mathbf{0})$} \\
\hline \multicolumn{2}{|c|}{} & & 1 & 2 & 3 & 4 & 5 \\
\cline { 3 - 8 } & $\emptyset_{\mathrm{ij}}(0)$ & 1 & 0 & 0 & 1 & 1 \\
\hline 1 & 1 & 1 & $\boldsymbol{k}_{\mathbf{0}}$ & 0 & 0 & $\boldsymbol{k}_{\mathbf{0}}$ & $\boldsymbol{k}_{\mathbf{0}}$ \\
\hline 2 & $\left(\frac{\mathrm{X}}{\mathrm{L}}\right)^{2}$ & 0 & 0 & 0 & 0 & 0 & 0 \\
\hline 3 & $\left(\frac{\mathrm{x}}{\mathrm{L}}\right)^{2}$ & 0 & 0 & 0 & 0 & 0 & 0 \\
\hline 4 & $\cos \frac{\pi \mathrm{x}}{\mathrm{L}}$ & 1 & $\boldsymbol{k}_{\mathbf{0}}$ & 0 & 0 & $\mathbf{k}_{\mathbf{0}}$ & $\mathbf{k}_{\mathbf{0}}$ \\
\hline 5 & $\cos \frac{2 \pi \mathrm{x}}{\mathrm{L}}$ & 1 & $\boldsymbol{k}_{\mathbf{0}}$ & 0 & 0 & $\boldsymbol{k}_{\mathbf{0}}$ & $\boldsymbol{k}_{\mathbf{0}}$ \\
\hline
\end{tabular}


Table 3-7: Clamped-Clamped (CC) Second Part of Eq.(3.17)

\begin{tabular}{|c|c|c|c|c|c|c|c|}
\hline & & & $k_{0} \varnothing$ & $(\boldsymbol{L}) \emptyset_{j}$ & & & \\
\hline & & & 1 & 2 & 3 & 4 & 5 \\
\hline & & & 1 & $\left(\frac{x}{I}\right)$ & $\left(\frac{x}{I}\right)^{2}$ & $\cos \frac{\pi x}{I}$ & $\cos \frac{2 \pi x}{I}$ \\
\hline & & $\emptyset_{i j}(L)$ & 1 & 1 & 1 & -1 & 1 \\
\hline 1 & 1 & 1 & $k_{0}$ & $k_{0}$ & $k_{0}$ & $-k_{0}$ & $k_{0}$ \\
\hline 2 & $\left(\frac{x}{I}\right)$ & 1 & $k_{0}$ & $k_{0}$ & $k_{0}$ & $-\boldsymbol{k}_{0}$ & $k_{0}$ \\
\hline 3 & $\left(\frac{x}{I}\right)^{2}$ & 1 & $k_{0}$ & $k_{0}$ & $k_{0}$ & $-k_{0}$ & $k_{0}$ \\
\hline 4 & $\cos \frac{\pi x}{L}$ & -1 & $-\boldsymbol{k}_{\mathbf{0}}$ & $-\boldsymbol{k}_{0}$ & $-\boldsymbol{k}_{0}$ & $k_{0}$ & $-k_{0}$ \\
\hline 5 & $\cos \frac{2 \pi x}{L}$ & 1 & $k_{0}$ & $k_{0}$ & $k_{0}$ & $-\boldsymbol{k}_{\mathbf{0}}$ & $k_{0}$ \\
\hline
\end{tabular}

Table 3-8: Clamped-Clamped (CC) Third Part of Eq.(3.17)

\begin{tabular}{|c|c|c|c|c|c|c|c|}
\hline \multicolumn{8}{|c|}{$\boldsymbol{k}_{r 0} L^{2} \emptyset_{i}^{\prime}(\mathbf{0}) \emptyset_{i}^{\prime}(\mathbf{0})$} \\
\hline & & & 1 & 2 & 3 & 4 & 5 \\
\hline & & & 0 & $\left(\frac{1}{2}\right)$ & $\left(\frac{2 x}{2}\right)$ & $-\frac{\pi}{I} \sin \frac{\pi x}{I}$ & $-\frac{2 \pi}{\pi} \sin \frac{2 \pi x}{\pi}$ \\
\hline & & $\emptyset_{i j}^{\prime}(0)$ & 0 & $\left(\frac{1}{L}\right)$ & 0 & 0 & 0 \\
\hline 1 & 0 & 0 & 0 & 0 & 0 & 0 & 0 \\
\hline 2 & $\left(\frac{1}{L}\right)$ & $\left(\frac{1}{L}\right)$ & 0 & $\boldsymbol{k}_{r 0}$ & 0 & 0 & 0 \\
\hline 3 & $\left(\frac{2 x}{L^{2}}\right)$ & 0 & 0 & 0 & 0 & 0 & 0 \\
\hline 4 & $-\frac{\pi}{L} \sin \frac{\pi x}{L}$ & 0 & 0 & 0 & 0 & 0 & 0 \\
\hline 5 & $-\frac{2 \pi}{L} \sin \frac{2 \pi x}{L}$ & 0 & 0 & 0 & 0 & 0 & 0 \\
\hline
\end{tabular}


Table 3-9: Clamped-Clamped (CC) Fourth Part of Eq.(3.17)

\begin{tabular}{|c|c|c|c|c|c|c|c|}
\hline \multicolumn{8}{|c|}{$\boldsymbol{k}_{r 0} L^{2} \emptyset_{i}^{\prime}(L) \emptyset_{j}^{\prime}(L)$} \\
\hline & & & 1 & 2 & 3 & 4 & 5 \\
\hline & & & 0 & $\left(\frac{1}{L}\right)$ & $\left(\frac{2 x}{L^{2}}\right)$ & $-\frac{\pi}{L} \sin \frac{\pi x}{L}$ & $-\frac{2 \pi}{L} \sin \frac{2 \pi x}{L}$ \\
\hline & & $\emptyset_{i j}^{\prime}(0)$ & 0 & $\left(\frac{1}{L}\right)$ & $\left(\frac{2}{L}\right)$ & 0 & 0 \\
\hline 1 & 0 & 0 & 0 & 0 & 0 & 0 & 0 \\
\hline 2 & $\left(\frac{1}{L}\right)$ & $\left(\frac{1}{L}\right)$ & 0 & $k_{r 0}$ & $2 k_{r 0}$ & 0 & 0 \\
\hline 3 & $\left(\frac{2 x}{L^{2}}\right)$ & 0 & 0 & $2 k_{r 0}$ & $4 k_{r 0}$ & 0 & 0 \\
\hline 4 & $-\frac{\pi}{L} \sin \frac{\pi x}{L}$ & 0 & 0 & 0 & 0 & 0 & 0 \\
\hline 5 & $-\frac{2 \pi}{L} \sin \frac{2 \pi x}{L}$ & 0 & 0 & 0 & 0 & 0 & 0 \\
\hline
\end{tabular}

It can be observed that a combination of the virtual linear and rotational springs at the both ends of the beam are used to represent a clamped-clamped condition. The penalty term matrix for clamped-clamped boundary condition is given by Eq.(3.20) as

$$
K_{P, C C}=\left[\begin{array}{ccccc}
2 k_{0} & k_{0} & k_{0} & 0 & 2 k_{0} \\
k_{0} & k_{0}+2 k_{r 0} & k_{0}+2 k_{r 0} & -k_{0} & k_{0} \\
k_{0} & k_{0}+2 k_{r 0} & k_{0}+4 k_{r 0} & -k_{0} & k_{0} \\
0 & -k_{0} & -k_{0} & 2 k_{0} & 0 \\
2 k_{0} & k_{0} & k_{0} & 0 & 2 k_{0}
\end{array}\right]
$$

Finally, to evaluate the natural frequency of a beam with different boundary conditions, the following condition must be satisfied,

$$
\left|K_{T}-\omega^{2} M\right|=0
$$

Where the total system stiffness matrix is given by

$$
K_{T}=K+K_{P}
$$

Eq.(3.22) shows how the different boundary conditions can be incorporated with the addition of appropriate $K_{P}$ matrix to the unconstrained beam stiffness matrix, $K$. 


\subsection{Numerical Simulations}

A detailed study is carried out to reveal the effect of penalty functions on predicting the eigenvalues and eigenvectors using the AAF method with penalties. Three boundary conditions are considered: Clamped-Free $(C F)$, Clamped-Clamped $(C C)$ and Simply-Supported (SS).

\subsubsection{Geometrical Parameters and Material Characteristics}

To carry out the detailed study, the following geometrical parameters and material characteristics are used, as tabulated in Table 3-10. The material for the beam is assumed to be Aluminium with Young's modulus $(E), 69.79 \mathrm{GPa}$ and mass density $(\rho), 2,600 \mathrm{~kg} / \mathrm{m}^{3}$.

Table 3-10: Geometrical Parameters \& Material Characteristics

\begin{tabular}{|l|c|}
\hline Length of the beam, $(L)$ (varying) & $0.5 \mathrm{~m}-1.5 \mathrm{~m}$ \\
\hline Thickness of the beam, $(h)$ (varying) & $3 \mathrm{~mm}-5 \mathrm{~mm}$ \\
\hline Width of the beam, $(b)$ (constant) & $50 \mathrm{~mm}$ \\
\hline Material & Aluminium \\
\hline Young's Modulus $(E)$ & $69.79 \mathrm{GPa}$ \\
\hline Mass Density $(\rho)$ & $2,600 \mathrm{~kg} / \mathrm{m}^{3}$ \\
\hline
\end{tabular}

The width of the beam is kept constant at $50 \mathrm{~mm}$ throughout the study. The length of the beam considered for the study is $0.5 \mathrm{~m}$ to $1.5 \mathrm{~m}$ in steps of $0.5 \mathrm{~m}$, and thickness $(h) 3 \mathrm{~mm}$ to $6 \mathrm{~mm}$ in steps of $1 \mathrm{~mm}$. The selection of the value of the virtual springs is one of the most crucial and challenging tasks in the use of $A A F$. In the present work, the values of virtual linear $\left(k_{0}\right)$ and rotational $\left(k_{r}\right)$ springs are taken as $1 \times 10^{10}$.The value is selected based on the range of values used in previous work [2],[11]. The maximum number of natural frequencies of interest is six and hence the number of terms, $N$, is taken to be 12 . Matlab code is developed to evaluate the natural 
frequencies and modeshapes, using both Euler-Bernoulli beam $(E B B)$ benchmark values and Alternative Admissible Function $(A A F)$ computed values. The percentage error and deviation of predicted natural frequencies (using the $E B B$ values as benchmarks) are calculated. EulerBernoulli Beam theory is known as classical beam theory, the values obtained through $E B B$ are considered to be true analytical values and are considered as benchmark for the following study. The predicted natural frequencies, percentage error and deviation, are tabulated in Table 3-11 to Table 3-19.

\subsubsection{Numerical Example on Clamped-Free $(C F)$ Beam}

For the Clamped-Free $(C F)$ beam boundary condition, it is noticed from Table 3-11 that the percentage error in predicting the natural frequency is less than $1 \%$. This indicates the effectiveness of the proposed $(A A F)$ method with penalties. It can be observed the largest frequency deviation of the predicted natural frequency at higher modes is $4.7567 \mathrm{~Hz}$, which is $0.6 \%$ error. This larger value of deviation maybe due to the selected value of penalty functions. In Figure 3-6, the dashed lines indicate the modeshapes obtained via Alternative Admissible Functions $(A A F)$, and the solid lines indicate the modeshapes of closed-form Euler-Bernoulli Beam. The modeshapes obtained using the $(A A F)$ show a good agreement with the Euler-Bernoulli Beam modeshapes. The first mode is indicated by blue colour, the second mode is indicated by green, red depicts the third mode of the beam, fourth mode of the beam is depicted by black colour, the fifth and sixth modes are represented by cyan and pink colours, respectively. The above stated legend applies to Figure 3-6Figure 3-14. 
Table 3-11: Natural Frequencies: $C F$ Beam, $L=0.5 \mathrm{~m}$, and $h=3 \mathrm{~mm}$

\begin{tabular}{|c|c|c|c|c|}
\hline Mode & $\begin{array}{c}\text { EBB } \\
\mathbf{( H z )}\end{array}$ & $\begin{array}{c}\text { AAF } \\
(\mathbf{H z})\end{array}$ & $\begin{array}{c}\text { \% } \\
\text { Error }\end{array}$ & Dev. \\
\hline 1st & 10.0432 & 10.0435 & 0.0030 & 0.0003 \\
\hline 2nd & 62.9396 & 62.9626 & 0.0365 & 0.0230 \\
\hline 3rd & 176.2326 & 176.4015 & 0.0959 & 0.1690 \\
\hline 4th & 345.3455 & 346.0410 & 0.2014 & 0.6955 \\
\hline 5th & 570.8811 & 572.6123 & 0.3032 & 1.7312 \\
\hline 6th & 852.7973 & 857.5540 & 0.5578 & 4.7567 \\
\hline
\end{tabular}

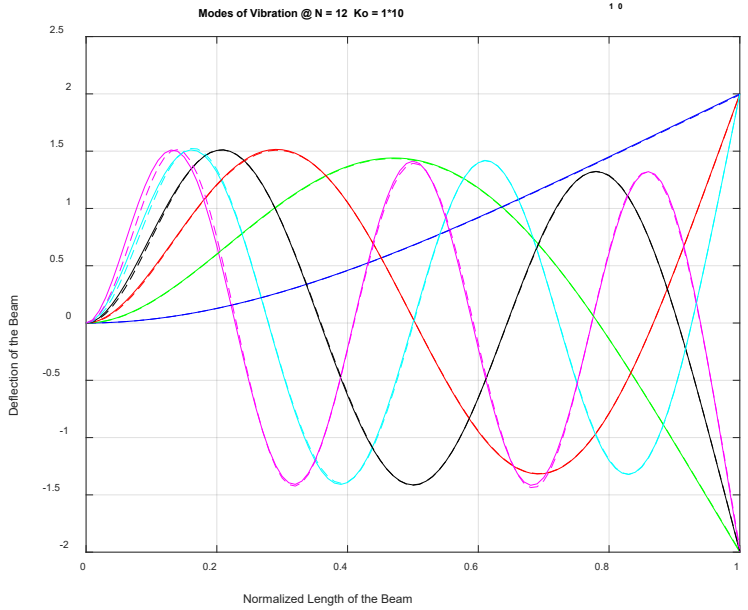

Figure 3-6: Modeshapes for $C F$ Beam, $L=0.5 \mathrm{~m}$, and $h=3 \mathrm{~mm}$

A similar type of trend is noticed in the results obtained for $C F$ boundary conditions, which are tabulated in Table 3-12. In this case, the length of the beam increased to $1 \mathrm{~m}$, keeping the thickness unchanged. It is noticed that the deviation in the predicted natural frequency at the $6^{\text {th }}$ mode is reduced to $1.1898 \mathrm{~Hz}(0.6 \%$ error). The modeshapes are shown in Figure 3-7

Table 3-12: Natural Frequencies: $C F$ Beam, $L=1 \mathrm{~m}$, and $h=3 \mathrm{~mm}$

\begin{tabular}{|c|r|r|r|c|}
\hline Mode & $\begin{array}{c}\text { EBB } \\
(\mathbf{H z})\end{array}$ & $\begin{array}{c}\text { AAF } \\
(\mathbf{H z})\end{array}$ & $\begin{array}{c}\text { \% } \\
\text { Error }\end{array}$ & Dev. \\
\hline $1^{\text {st }}$ & 2.5108 & 2.5109 & 0.0030 & 0.0001 \\
\hline $2^{\text {nd }}$ & 15.7349 & 15.7406 & 0.0366 & 0.0058 \\
\hline $3^{\text {rd }}$ & 44.0581 & 44.1004 & 0.0959 & 0.0423 \\
\hline $4^{\text {th }}$ & 86.3364 & 86.5104 & 0.2015 & 0.1740 \\
\hline $5^{\text {th }}$ & 142.7203 & 143.1534 & 0.3035 & 0.4331 \\
\hline $6^{\text {th }}$ & 213.1993 & 214.3892 & 0.5550 & 1.1898 \\
\hline
\end{tabular}




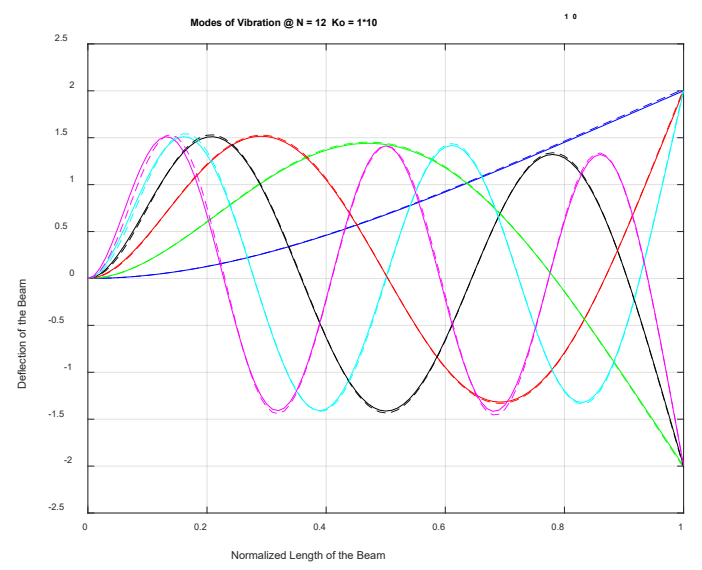

Figure 3-7: Modeshapes for $C F$ Beam, $L=1 \mathrm{~m}$, and $h=3 \mathrm{~mm}$

Table 3-13 indicates the results obtained for $C F$ boundary conditions with the length of the beam $1.5 \mathrm{~m}$ and thickness $3 \mathrm{~mm}$. The percentage error of the predicted natural frequency is less than $1 \%$. This suggests with the change in geometrical properties of the beam for $C F$ boundary conditions, the AAF method provides reliable results. The deviation in the predicted value of natural frequency for the $6^{\text {th }}$ mode is further reduced to $0.5288 \mathrm{~Hz}(0.6 \%)$. The percentage error is in same range and therefore the deviation in the natural frequency is acceptable. This indicates that the deviation of the predicted natural frequency has changed with the change in geometrical parameters. Further, a trend of decreases in deviation in the values of natural frequencies is observed as the length of the beam increases from $L=0.5$ to $L=1.5 \mathrm{~m}$. The modeshapes are shown in Figure 3-8.

Table 3-13: Natural Frequencies: $C F$ Beam, $L=1.5 \mathrm{~m}$, and $h=3 \mathrm{~mm}$

\begin{tabular}{|c|c|c|c|c|}
\hline Mode & $\begin{array}{c}\text { EBB } \\
(\mathbf{H z})\end{array}$ & $\begin{array}{c}\text { AAF } \\
(\mathbf{H z})\end{array}$ & $\begin{array}{c}\text { \% } \\
\text { Error }\end{array}$ & Dev. \\
\hline $1^{\text {st }}$ & 1.1159 & 1.1159 & 0.0030 & 0.0000 \\
\hline $2^{\text {nd }}$ & 6.9933 & 6.9958 & 0.0366 & 0.0026 \\
\hline $3^{\text {rd }}$ & 19.5814 & 19.6002 & 0.0960 & 0.0188 \\
\hline $4^{\text {th }}$ & 38.3717 & 38.4491 & 0.2015 & 0.0773 \\
\hline $5^{\text {th }}$ & 63.4312 & 63.6237 & 0.3035 & 0.1925 \\
\hline $6^{\text {th }}$ & 94.7553 & 95.2841 & 0.5581 & 0.5288 \\
\hline
\end{tabular}




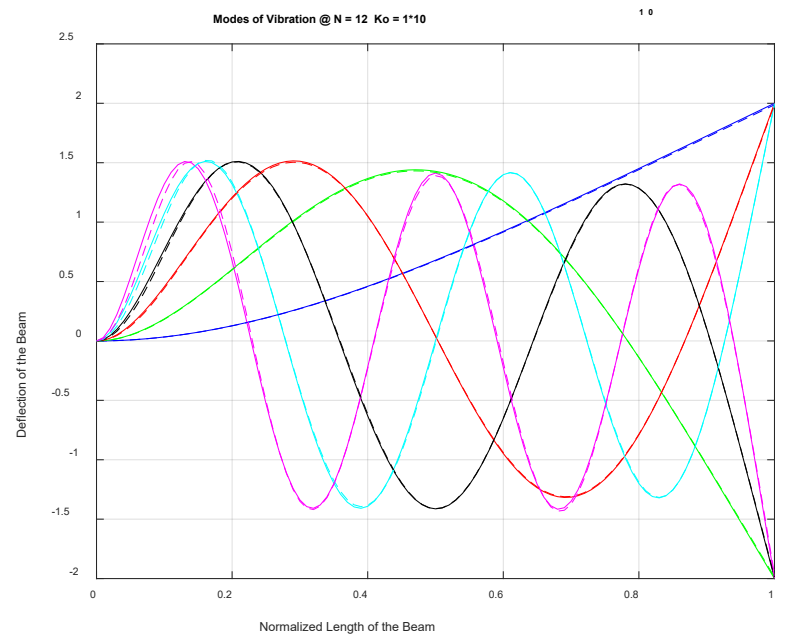

Figure 3-8: Modeshapes for $C F$ Beam, $L=1.5 \mathrm{~m}$, and $h=3 \mathrm{~mm}$

In Table 3-11,Table 3-12, and Table 3-13 the highlighted value of deviation is decreasing as the length of the beam increases. It can be observed that the assumed value of penalty function for beams with lower stiffness yields lower deviation while the deviation in predicting the natural frequency for beams with higher stiffness has a higher deviation. This leads to scope for selection of appropriate penalty values for beams of different lengths to give good agreement between the Euler-Bernoulli values and the $A A F$ method.

\subsubsection{Numerical Example on Simply-Supported (SS) Beam}

In the case of simply-supported $(S S)$ beam boundary conditions, the predicted natural frequencies are anticipated to fall between $C F$ and $C C$ boundary cases.

Table 3-14: Natural Frequencies: SS Beam, $L=0.5 \mathrm{~m}$, and $h=3 \mathrm{~mm}$

\begin{tabular}{|c|c|c|c|c|}
\hline Mode & $\begin{array}{c}\text { EBB } \\
(\mathbf{H z})\end{array}$ & $\begin{array}{c}\mathbf{A A F} \\
\mathbf{( H z )}\end{array}$ & $\begin{array}{c}\text { \% } \\
\text { Error }\end{array}$ & Dev. \\
\hline $1^{\text {st }}$ & 28.1920 & 28.1970 & 0.0183 & 0.0052 \\
\hline $2^{\text {nd }}$ & 112.7700 & 112.8300 & 0.0555 & 0.0626 \\
\hline $3^{\text {rd }}$ & 253.7200 & 254.2000 & 0.1854 & 0.4705 \\
\hline $4^{\text {th }}$ & 451.0700 & 452.2200 & 0.2561 & 1.1552 \\
\hline $5^{\text {th }}$ & 704.7900 & 709.4300 & 0.6589 & 4.6436 \\
\hline $6^{\text {th }}$ & 1014.9000 & 1022.5000 & 0.7458 & 7.5690 \\
\hline
\end{tabular}




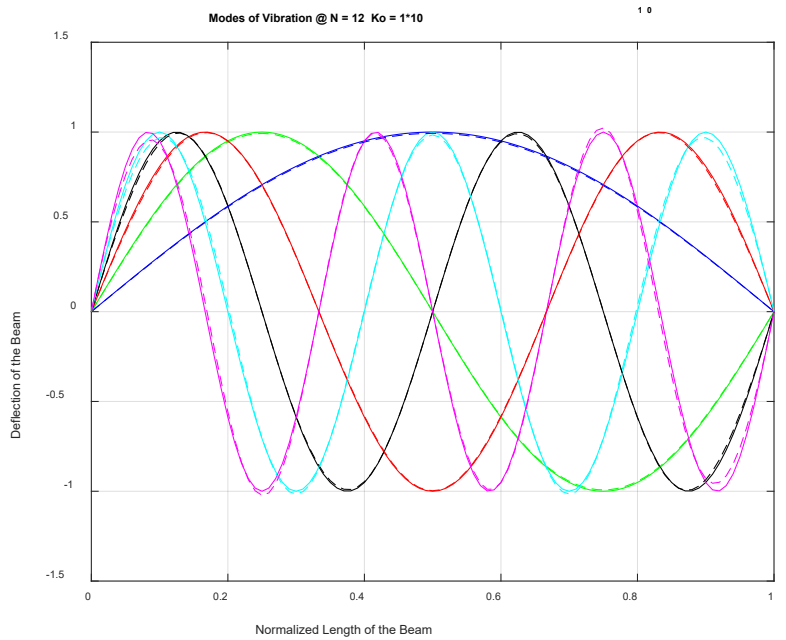

Figure 3-9: Modeshapes for SS Beam, $L=0.5 \mathrm{~m}$, and $h=3 \mathrm{~mm}$

Table 3-14, Table 3-15, and Table 3-16 depict the results obtained for SS boundary conditions for lengths $L=0.5 \mathrm{~m}, 1 \mathrm{~m}$, and $1.5 \mathrm{~m}$, and the modeshapes are shown in Figure 3-9, Figure 3-10, and Figure 3-11, respectively. It can be noticed that the deviation in the predicted natural frequencies has a decreasing trend as observed in the $C F$ boundary conditions. A maximum deviation of 7.5690 $\mathrm{Hz}$, to $0.8415 \mathrm{~Hz}$, is observed for the $6^{\text {th }}$ mode for the Simply-Supported conditions, which indicates that as the length of beam is increased, the deviation of the natural frequency decreases. Traditionally, the assumed mode method overestimates the stiffness of the system. This might also suggest that the value of penalties required for the stiffer beams may be higher than the selected value of penalties.

Table 3-15: Natural Frequencies: $S S$ Beam, $L=1 \mathrm{~m}$, and $h=3 \mathrm{~mm}$

\begin{tabular}{|c|c|c|c|c|}
\hline Mode & $\begin{array}{c}\text { EBB } \\
(\mathbf{H z})\end{array}$ & $\begin{array}{c}\text { AAF } \\
(\mathbf{H z})\end{array}$ & $\begin{array}{c}\text { \% } \\
\text { Error }\end{array}$ & Dev. \\
\hline $1^{\text {st }}$ & 7.0479 & 7.0492 & 0.0184 & 0.0013 \\
\hline $2^{\text {nd }}$ & 28.1920 & 28.2070 & 0.0555 & 0.0157 \\
\hline $3^{\text {rd }}$ & 63.4310 & 63.5490 & 0.1855 & 0.1177 \\
\hline $4^{\text {th }}$ & 112.7700 & 113.0600 & 0.2563 & 0.2890 \\
\hline $5^{\text {th }}$ & 176.2000 & 177.3600 & 0.6591 & 1.1614 \\
\hline $6^{\text {th }}$ & 253.7200 & 255.6200 & 0.7462 & 1.8932 \\
\hline
\end{tabular}




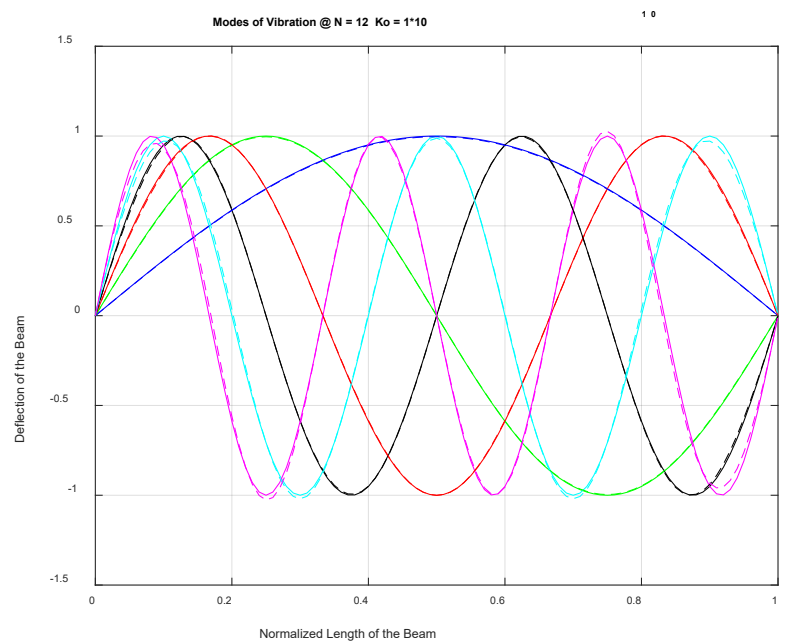

Figure 3-10: Modeshapes for $S S$ Beam, $L=1 \mathrm{~m}$, and $h=3 \mathrm{~mm}$

Table 3-16: Natural Frequencies: $S S$ Beam, $L=1.5 \mathrm{~m}$, and $h=3 \mathrm{~mm}$

\begin{tabular}{|c|c|c|c|c|}
\hline Mode & $\begin{array}{c}\text { EBB } \\
(\mathbf{H z})\end{array}$ & $\begin{array}{c}\text { AAF } \\
\mathbf{( H z )}\end{array}$ & $\begin{array}{c}\text { \% } \\
\text { Error }\end{array}$ & Dev. \\
\hline $1^{\text {st }}$ & 3.1324 & 3.1330 & 0.0184 & 0.0006 \\
\hline $2^{\text {nd }}$ & 12.5300 & 12.5370 & 0.0555 & 0.0070 \\
\hline $3^{\text {rd }}$ & 28.1920 & 28.2440 & 0.1855 & 0.0523 \\
\hline $4^{\text {th }}$ & 50.1180 & 50.2470 & 0.2563 & 0.1285 \\
\hline $5^{\text {th }}$ & 78.3100 & 78.8260 & 0.6592 & 0.5162 \\
\hline $6^{\text {th }}$ & 112.7700 & 113.6100 & 0.7462 & 0.8415 \\
\hline
\end{tabular}

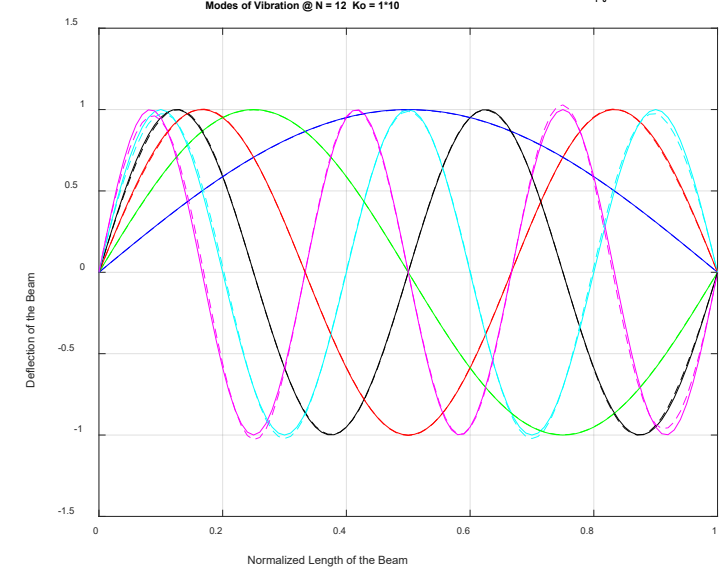

Figure 3-11: Modeshapes for SS Beam, $L=1.5 \mathrm{~m}$, and $h=3 \mathrm{~mm}$ The study is carried out for $C C$ boundary condition in the following section. 


\subsubsection{Numerical Example on Clamped-Clamped (CC) Beam}

Further, the validation of the $A A F$ method is carried out for Clamped-Clamped condition and is discussed below.

Table 3-17: Natural Frequencies: $C C$ Beam, $L=0.5 \mathrm{~m}$, and $h=3 \mathrm{~mm}$

\begin{tabular}{|c|c|c|c|r|}
\hline Mode & $\begin{array}{c}\text { EBB } \\
(\mathbf{H z})\end{array}$ & $\begin{array}{c}\text { AAF } \\
(\mathbf{H z})\end{array}$ & $\begin{array}{c}\text { \% } \\
\text { Error }\end{array}$ & Dev. \\
\hline $1^{\text {st }}$ & 63.9070 & 63.9580 & 0.0792 & 0.0506 \\
\hline $2^{\text {nd }}$ & 176.1600 & 176.4600 & 0.1659 & 0.2922 \\
\hline $3^{\text {rd }}$ & 345.3500 & 346.8900 & 0.4450 & 1.5367 \\
\hline $4^{\text {th }}$ & 570.8800 & 573.9600 & 0.5398 & 3.0815 \\
\hline $5^{\text {th }}$ & 852.8000 & 862.3900 & 1.1253 & 9.5969 \\
\hline $6^{\text {th }}$ & 1191.1000 & 1204.9000 & 1.1602 & 13.8190 \\
\hline
\end{tabular}

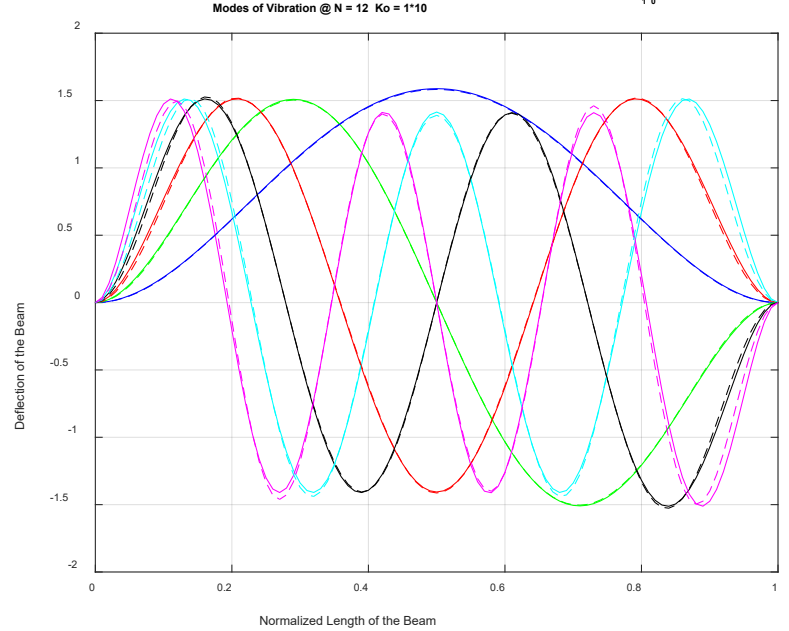

Figure 3-12: Modeshapes for $C C$ Beam, $L=0.5 \mathrm{~m}$, and $h=3 \mathrm{~mm}$

In Table 3-17, the boundary condition is considered as Clamped-Clamped $(C C)$, with length of the beam $L=0.5 \mathrm{~mm}$ and thickness $h=3 \mathrm{~mm}$. The percentage error and the deviation of natural frequency are tabulated in Table 3-17. Compared to the Clamped-Free $(C F)$ boundary condition, the deviation of the predicted values of natural frequencies for Clamped-Clamped $(C C)$ boundary condition is larger. It can be noticed that at the $6^{\text {th }}$ mode, the deviation of natural frequency is $13.8190 \mathrm{~Hz}(1.2 \%)$. 
Table 3-18: Natural Frequencies: $C C$ Beam, $L=1 \mathrm{~m}$, and $h=3 \mathrm{~mm}$

\begin{tabular}{|l|l|l|l|l|}
\hline Mode & $\begin{array}{c}\text { EBB } \\
(\mathbf{H z})\end{array}$ & $\begin{array}{c}\text { AAF } \\
(\mathbf{H z})\end{array}$ & $\begin{array}{c}\% \\
\text { Error }\end{array}$ & Dev. \\
\hline $1^{\text {st }}$ & 15.9768 & 15.9895 & 0.0792 & 0.0127 \\
\hline $2^{\text {nd }}$ & 44.0407 & 44.1138 & 0.1660 & 0.0731 \\
\hline $3^{\text {rd }}$ & 86.3374 & 86.7218 & 0.4452 & 0.3844 \\
\hline $4^{\text {th }}$ & 142.7202 & 143.4912 & 0.5402 & 0.7710 \\
\hline $5^{\text {th }}$ & 213.1993 & 215.5999 & 1.1260 & 2.4005 \\
\hline $6^{\text {th }}$ & 297.7742 & 301.2314 & 1.1610 & 3.4572 \\
\hline
\end{tabular}

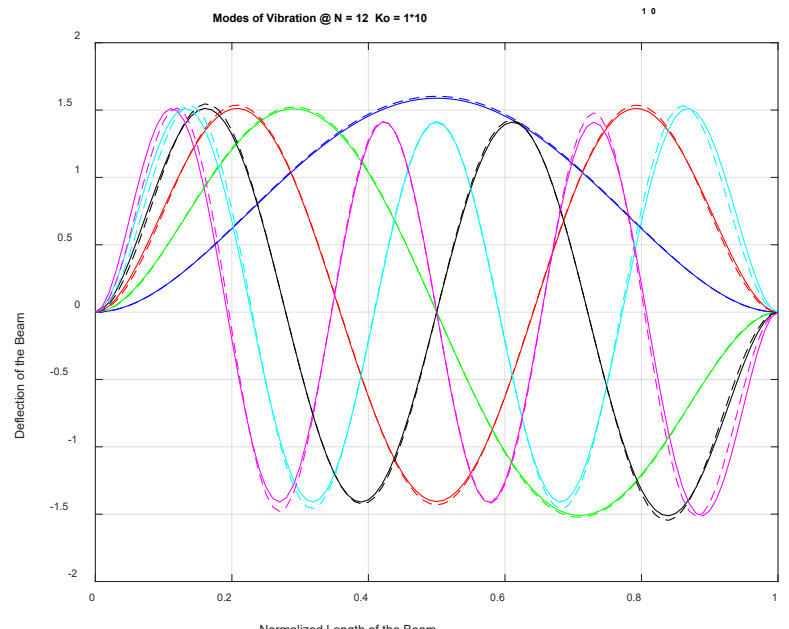

Figure 3-13: Modeshapes for $C C$ Beam, $L=1 \mathrm{~m}$, and $h=3 \mathrm{~mm}$.

A similar trend was observed in the case of Clamped-Free boundary condition, a higher value of deviation for higher modes. In Table 3-18, as the length of the beam is increased to $1 \mathrm{~m}$, the stiffness of the system decreases as the natural frequencies of the system are observed to drop. The deviation of the natural frequencies shows a decrease in value compared to the beam with length $0.5 \mathrm{~m}$ and thickness $3 \mathrm{~mm}$ for the Clamped-Clamped beam. Further decreases in the predicted value of natural frequencies of the system are observed in Table 3-19. The deviation of the natural frequency at the $6^{\text {th }}$ mode is $1.5367 \mathrm{~Hz}(1.2 \%)$. The higher deviation in the predicted natural frequency provides scope to determine an appropriate value for penalties with changes in boundary conditions and the geometrical parameters. The first six mode shapes of a $C C$ beam are shown in Figure 3-12, Figure 
3-13 and Figure 3-14 for lengths $L=0.5 \mathrm{~m}, L=1 \mathrm{~m}$, and $L=1.5 \mathrm{~m}$, respectively and thickness $h$ $=3 \mathrm{~mm}$.

Table 3-19: Natural Frequencies: $C C$ Beam, $L=1.5 \mathrm{~m}$, and $h=3 \mathrm{~mm}$

\begin{tabular}{|c|c|c|c|c|}
\hline Mode & $\begin{array}{c}\text { EBB } \\
(\mathbf{H z})\end{array}$ & $\begin{array}{c}\text { AAF } \\
(\mathbf{H z})\end{array}$ & $\begin{array}{c}\text { \% } \\
\text { Error }\end{array}$ & Dev. \\
\hline $1^{\text {st }}$ & 7.1008 & 7.1064 & 0.0792 & 0.0056 \\
\hline $2^{\text {nd }}$ & 19.5737 & 19.6062 & 0.1661 & 0.0325 \\
\hline $3^{\text {rd }}$ & 38.3722 & 38.5431 & 0.4453 & 0.1709 \\
\hline $4^{\text {th }}$ & 63.4312 & 63.7739 & 0.5403 & 0.3427 \\
\hline $5^{\text {th }}$ & 94.7553 & 95.8222 & 1.1260 & 1.0670 \\
\hline $6^{\text {th }}$ & 132.3441 & 133.8808 & 1.1611 & 1.5367 \\
\hline
\end{tabular}

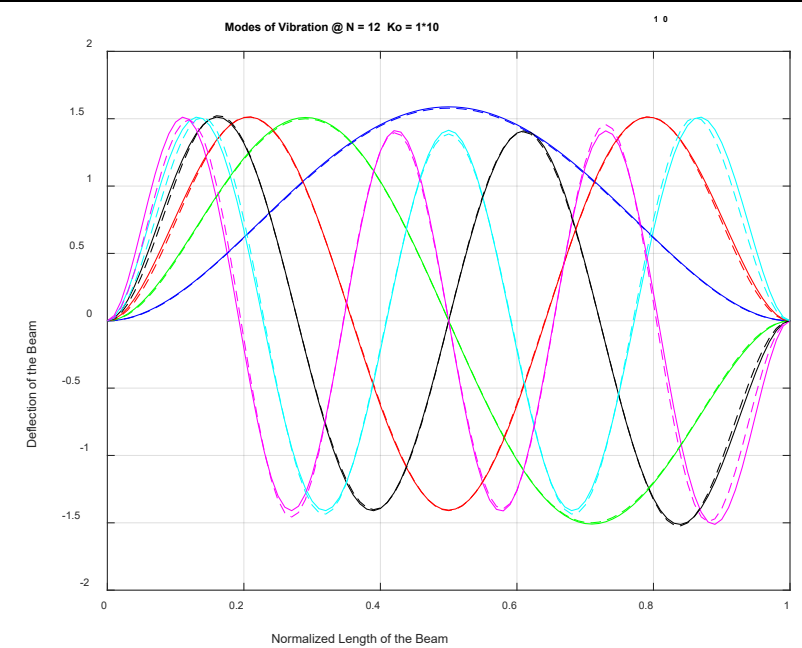

Figure 3-14: Modeshapes for $C C$ Beam, $L=1.5 \mathrm{~m}$, and $h=3 \mathrm{~mm}$

For a given fixed dimension and material property, the present method shows a similar trend compared to Euler-Bernoulli in predicting the natural frequencies i.e., the natural frequency of the system for the $S S$ boundary condition is higher than the $C F$ boundary condition and less than the $C C$ boundary condition as observed in the natural frequencies of an Euler-Bernoulli beam.

As a conclusion on results obtained in sections 3.7.2, 3.7.3 and 3.7.4, it is noticed that the trend of natural frequencies obtained for Clamped-Clamped (CC) boundary condition is larger than those obtained for Simply-Supported (SS) boundary condition, which in turn is larger than those obtained 
for Clamped-Free $(C F)$ boundary condition. This trend of increase in natural frequencies similar to the one obtained in Euler-Bernoulli beam.

\subsection{Numerical Example on Varying Thickness for All Three Boundary}

\section{Conditions}

A similar evaluation is carried out by keeping the length constant and varying only the thickness of the beam from $3 \mathrm{~mm}$ to $5 \mathrm{~mm}$ in a step of $1 \mathrm{~mm}$, for all three boundary conditions. The number of terms $N$ and value for penalty functions are kept unchanged. Only the predicted natural frequencies obtained by $A A F$ method compared to closed-form Euler-Bernoulli beam values are tabulated as one table for each boundary conditions. Table 3-20 and Table 3-21 indicates the tabulated results for Clamped-Free boundary conditions.

Table 3-20: Natural Frequencies: $C F$ Beam, $L=1 \mathrm{~m}$, and $h=3 \mathrm{~mm}$ and $4 \mathrm{~mm}$

\begin{tabular}{|c|r|r|c|c|}
\hline Mode & $\begin{array}{c}\text { EBB } \\
(\mathbf{H z})\end{array}$ & $\begin{array}{c}\text { AAF } \\
(\mathbf{H z})\end{array}$ & $\begin{array}{c}\text { \% } \\
\text { Error }\end{array}$ & Dev \\
\hline $\boldsymbol{h}=\mathbf{3} \mathbf{~ m m}$ \\
\hline 1 & 2.5108 & 2.5109 & 0.0030 & 0.0001 \\
\hline 2 & 15.7349 & 15.7406 & 0.0366 & 0.0058 \\
\hline 3 & 44.0581 & 44.1004 & 0.0959 & 0.0423 \\
\hline 4 & 86.3364 & 86.5104 & 0.2015 & 0.1740 \\
\hline 5 & 142.7203 & 143.1534 & 0.3035 & 0.4331 \\
\hline 6 & 213.1993 & 214.3892 & 0.5581 & 1.1898 \\
\hline $\boldsymbol{h}=\mathbf{4} \mathbf{m m}$ & & & \\
\hline 1 & 3.3477 & 3.3478 & 0.0030 & 0.0001 \\
\hline 2 & 20.9799 & 20.9875 & 0.0366 & 0.0077 \\
\hline 3 & 58.7442 & 58.8005 & 0.0959 & 0.0564 \\
\hline 4 & 115.1152 & 115.3471 & 0.2015 & 0.2320 \\
\hline 5 & 190.2937 & 190.8711 & 0.3034 & 0.5774 \\
\hline 6 & 284.2658 & 285.8520 & 0.5580 & 1.5863 \\
\hline
\end{tabular}


Table 3-21: Natural Frequencies: CF Beam, $L=1 \mathrm{~m}$, and $h=5 \mathrm{~mm}$

\begin{tabular}{|c|r|r|r|c|}
\hline Mode & $\begin{array}{c}\text { EBB } \\
(\mathbf{H z})\end{array}$ & $\begin{array}{c}\text { AAF } \\
(\mathbf{H z})\end{array}$ & $\begin{array}{c}\text { \% } \\
\text { Error }\end{array}$ & Dev \\
\hline $\boldsymbol{h}=\mathbf{5} \mathbf{~ m m}$ \\
\hline 1 & 4.1847 & 4.1848 & 0.0030 & 0.0001 \\
\hline 2 & 26.2248 & 26.2344 & 0.0366 & 0.0096 \\
\hline 3 & 73.4302 & 73.5007 & 0.0959 & 0.0704 \\
\hline 4 & 143.8940 & 144.1839 & 0.2015 & 0.2899 \\
\hline 5 & 237.8671 & 238.5887 & 0.3034 & 0.7216 \\
\hline 6 & 355.3322 & 357.3147 & 0.5579 & 1.9825 \\
\hline
\end{tabular}

Keeping all the other parameters unchanged, as the thickness of the beam increases the stiffness of the beam increases, which in turn increases the natural frequencies of the beam. Even though the percentage of error in predicting the natural frequencies is less than $1 \%$, the deviation of natural frequency at $6^{\text {th }}$ mode is more than $1 \mathrm{~Hz}$. Variation in the deviation of natural frequencies for different thicknesses of the beam is a clear indication of the assumed value for penalty functions. As expected, an increasing trend in deviation value in the predicted natural frequencies is noticed.

Table 3-22: Natural Frequencies: $C C$ Beam, $\mathrm{L}=1 \mathrm{~m}$, and Varying Thickness $(h)$ shows the results for Clamped-Clamped boundary conditions. It is noticed that there is an increase in deviation in the predicted values of natural frequencies. At higher modes, the deviation of natural frequency is from $3.4572 \mathrm{~Hz}$ to $5.7598 \mathrm{~Hz}$, with increase in thickness. 
Table 3-22: Natural Frequencies: $C C$ Beam, $\mathrm{L}=1 \mathrm{~m}$, and Varying Thickness $(h)$

\begin{tabular}{|c|r|r|c|c|}
\hline Mode & $\begin{array}{c}\text { EBB } \\
(\mathbf{H z})\end{array}$ & $\begin{array}{c}\text { AAF } \\
\text { (Hz) }\end{array}$ & $\begin{array}{c}\text { \% } \\
\text { Error }\end{array}$ & Dev \\
\hline $\boldsymbol{h}=\mathbf{3} \mathbf{~ m m}$ \\
\hline 1 & 15.9768 & 15.9895 & 0.0792 & 0.0127 \\
\hline 2 & 44.0407 & 44.1138 & 0.1660 & 0.0731 \\
\hline 3 & 86.3374 & 86.7218 & 0.4452 & 0.3844 \\
\hline 4 & 142.7202 & 143.4912 & 0.5402 & 0.7710 \\
\hline 5 & 213.1993 & 215.5999 & 1.1260 & 2.4005 \\
\hline 6 & 297.7742 & 301.2314 & 1.1610 & 3.4572 \\
\hline $\boldsymbol{h}=\mathbf{4} \mathbf{m m}$ & 21.3024 & 21.3193 & 0.0792 & 0.0169 \\
\hline 1 & \multicolumn{5}{|c|}{} \\
\hline 2 & 58.7210 & 58.8184 & 0.1660 & 0.0975 \\
\hline 3 & 115.1166 & 115.6291 & 0.4452 & 0.5125 \\
\hline 4 & 190.2936 & 191.3214 & 0.5401 & 1.0278 \\
\hline 5 & 284.2658 & 287.4662 & 1.1258 & 3.2004 \\
\hline 6 & 397.0323 & 401.6413 & 1.1608 & 4.6089 \\
\hline $\boldsymbol{h}=\mathbf{5} \mathbf{m m}$ & \multicolumn{5}{|l}{} \\
\hline 1 & 26.6280 & 26.6491 & 0.0792 & 0.0211 \\
\hline 2 & 73.4012 & 73.5230 & 0.1659 & 0.1218 \\
\hline 3 & 143.8957 & 144.5362 & 0.4451 & 0.6405 \\
\hline 4 & 237.8669 & 239.1514 & 0.5400 & 1.2845 \\
\hline 5 & 355.3322 & 359.3320 & 1.1256 & 3.9998 \\
\hline 6 & 496.2904 & 502.0502 & 1.1606 & 5.7598 \\
\hline
\end{tabular}

The deviation and the percentage of error for natural frequencies for beams with varying thickness for the Simply-Supported boundary condition is tabulated in Table 3-23. It is noticed that the deviation in the predicted values for natural frequencies by AAF method lies between the $C F$ and $C C$ boundary conditions.

The value of penalty considered in the study was $1 \times 10^{10}$ and the length and thickness of the beam was varied. Varying the length and thickness of the beam individually demonstrates excellent results. The assumed values for penalty functions yield a good verification for predicted 
natural frequencies and modeshapes and provide a scope to determine the appropriate value of penalties for different geometrical parameters. It indicates a trend with changes in stiffness of the beam for the assumed fixed value of penalty terms.

Table 3-23: Natural Frequencies: SS Beam, $L=1 \mathrm{~m}$, and Varying Thickness $(h)$

\begin{tabular}{|c|c|c|c|c|}
\hline Mode & $\begin{array}{l}\text { EBB } \\
(\mathrm{Hz})\end{array}$ & $\begin{array}{l}\mathbf{A A F} \\
(\mathbf{H z})\end{array}$ & $\begin{array}{c}\% \\
\text { Error }\end{array}$ & Dev \\
\hline \multicolumn{5}{|c|}{$h=3 \mathrm{~mm}$} \\
\hline 1 & 7.0479 & 7.0492 & 0.0184 & 0.0013 \\
\hline 2 & 28.1916 & 28.2073 & 0.0555 & 0.0157 \\
\hline 3 & 63.4312 & 63.5489 & 0.1855 & 0.1177 \\
\hline 4 & 112.7666 & 113.0556 & 0.2563 & 0.2890 \\
\hline 5 & 176.1978 & 177.3592 & 0.6591 & 1.1614 \\
\hline 6 & 253.7248 & 255.6180 & 0.7462 & 1.8932 \\
\hline \multicolumn{5}{|c|}{$h=4 \mathrm{~mm}$} \\
\hline 1 & 9.3972 & 9.3989 & 0.0184 & 0.0017 \\
\hline 2 & 37.5889 & 37.6097 & 0.0555 & 0.0209 \\
\hline 3 & 84.5749 & 84.7318 & 0.1855 & 0.1569 \\
\hline 4 & 150.3554 & 150.7407 & 0.2562 & 0.3853 \\
\hline 5 & 234.9304 & 236.4788 & 0.6591 & 1.5484 \\
\hline 6 & 338.2997 & 340.8238 & 0.7461 & 2.5240 \\
\hline \multicolumn{5}{|c|}{$h=5 \mathrm{~mm}$} \\
\hline 1 & 11.7465 & 11.7487 & 0.0184 & 0.0022 \\
\hline 2 & 46.9861 & 47.0122 & 0.0555 & 0.0261 \\
\hline 3 & 105.7187 & 105.9148 & 0.1855 & 0.1961 \\
\hline 4 & 187.9443 & 188.4258 & 0.2562 & 0.4815 \\
\hline 5 & 293.6630 & 295.5982 & 0.6590 & 1.9352 \\
\hline 6 & 422.8747 & 426.0292 & 0.7460 & 3.1545 \\
\hline
\end{tabular}

To validate the change in deviation in natural frequency, the value of penalty terms is varied from $1 \times 10^{6}$ to $1 \times 10^{12}$. The geometrical parameters of the beam are length $(L) 1 \mathrm{~m}$, thickness $(h) 5 \mathrm{~mm}$ and width $50 \mathrm{~mm}$. Table 3-24 tabulates the deviation of the first six natural frequencies with varying penalty term values. 
Table 3-24: Deviations in Predicted Natural Frequencies $(\mathrm{Hz})$ with Varying Penalty Value

\begin{tabular}{|c|r|r|r|r|}
\hline \multirow{2}{*}{ Mode } & \multicolumn{4}{|c|}{$\boldsymbol{k}_{\boldsymbol{o}}=\boldsymbol{k}_{\boldsymbol{r} \boldsymbol{o}}=\mathbf{1 \times 1 0} \mathbf{}$} \\
\cline { 2 - 5 } & $\mathbf{p}=\mathbf{6}$ & $\mathbf{p}=7$ & $\mathbf{p}=\mathbf{8}$ & $\mathbf{p}=\mathbf{9}$ \\
\hline $1^{\text {st }}$ & -0.00627 & 0.001313 & 0.002072 & 0.002148 \\
\hline $2^{\text {nd }}$ & -0.10872 & 0.01261 & 0.02475 & 0.025964 \\
\hline $3^{\text {rd }}$ & -0.48851 & 0.127924 & 0.189333 & 0.195471 \\
\hline $4^{\text {th }}$ & -1.70133 & 0.266455 & 0.460223 & 0.479568 \\
\hline $5^{\text {th }}$ & -3.48424 & 1.412468 & 1.883609 & 1.930529 \\
\hline $6^{\text {th }}$ & -8.43147 & 2.072766 & 3.048119 & 3.144865 \\
\hline
\end{tabular}

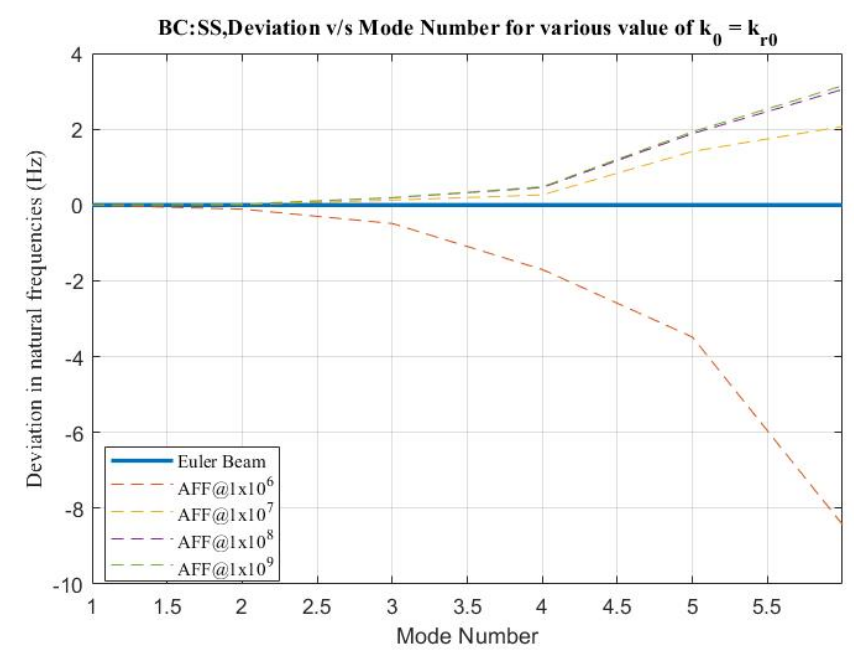

Figure 3-15: Deviation v/s Model Number for Various Penalty Values

Table 3-24 indicates that the assumed values for the penalty terms is not equally good for different stiffness and boundaries. To understand the trend, Figure 3-15 depicts the deviation of natural frequency of AAF method from closed-form Euler- Bernoulli Beam $(E B B)$ method for a SimplySupported beam with $L=1 \mathrm{~m}, h=5 \mathrm{~mm}$, and width $=50 \mathrm{~mm}$.

As the value of the power for penalty term decreases, the deviation in natural frequency decreases. A decrease in power to 6 gives a negative deviation, indicating a suitable value for Simply-Supported beam with above dimensions is between power 6 and 7 . 


\subsection{Conclusions}

In the present study, the Alternative Admissible Functions method with penalties is studied for varying geometrical parameters and boundary conditions. The natural frequencies and the mode shapes obtained using the $A A F$ method with penalties are in excellent agreement with the benchmark closed-form Euler-Bernoulli values with less than $1 \%$ error in predicting the natural frequency of $C F$ and $S S$ boundary conditions and less than $2 \%$ error in predicting the natural frequency for $C C$ boundary condition. Stable modeshapes for all three-boundary condition are observed. For the cases considered in the study, it is found that an increase in the length of the beam caused a decrease in the deviation in the natural frequency. It is also found that a decrease in the thickness of the beam indicated a decrease in deviation of predicted natural frequency. This demonstrates that the selected values of penalties need to be in agreement with the underlying beam properties, as well as the boundary conditions of the beam. It is observed that the selected value of penalties works well with beams with lower stiffness but produces a higher deviation for beams with higher stiffness for different boundary conditions. The value of the penalties not only depend on the type of boundary conditions, but also demonstrate an apparent relation between the penalty stiffnesses and the stiffness of the beam. This provides future scope for appropriate selection of the penalty values depending on the boundary conditions and geometrical beam properties. 


\section{Selection and Validation of Penalty Values for Alternative}

\section{Admissible Function}

\subsection{Introduction}

The most important factor in determining the shape of an eigenfunction is the boundary condition associated with the problem. Depending on its type, a boundary condition can render a specific eigenfunction numerically unstable or unable to be found in closed form, which further complicates the solution process.

The penalty function approach is an alternative method to the traditional way of solving the transverse vibrations of a beam via assumed modes by replacing each boundary condition with a set of virtual linear and rotational springs. The potential energy terms associated with these springs modifies the stiffness matrix and is introduced into the Rayleigh quotient. The final step in developing the boundary conditions via the penalty function approach is to assign a specific value to the stiffness per degree of freedom at the boundary.

The assumed modes approach in Chapter 3 demonstrated the effectiveness of the alternative admissible functions with penalties, however, to date there exist no guidelines for the selection of the appropriate penalty function value for specific beam properties and boundary conditions. It was observed in Chapter 3 that the selected value of penalties worked well with beams with lower stiffness values but produced higher deviations for beams with higher stiffness, for the boundary conditions under consideration. The value of the penalties not only depend on the type of boundary conditions, but there may be an apparent relation between the penalty function and the system parameters. This chapter reports on an approach to establish a general relation between the selection of penalty functions and the system parameters. The chapter starts with the penalty functions for different boundary conditions, and then continues with a mathematical 
procedure to obtain the relationship between the penalty functions and system parameters for the boundary conditions under consideration, viz., Clamped-Free $(C F)$, Simply-Supported (SS) and Clamped-Clamped $(C C)$ boundary conditions. A generalized methodology for all boundary conditions is explained with the help of a flow-chart. Obtained expressions for different boundary conditions are validated with the numerical results obtained from classic closed-form solutions.

\subsection{Penalty Function for Different Boundary Conditions}

In the present study, it is proposed that the virtual linear and rotational springs of the penalties can be written as

$$
k_{r 0}=k_{0}=\frac{E I}{L^{3}} k
$$

where $E, I, L$ are the known beam properties (Young's modulus, area moment of inertia and length) and $k$ is the values to be selected to represent those virtual springs. The assumption is made to encapsulate the beam properties based on the conclusions of Chapter 3 .

From Euler-Bernoulli beam theory [1], it is known that the natural frequencies can be written as

$$
\omega_{n}^{2}=\left(\beta_{n} L\right)^{4} \frac{E I}{\rho L^{4}}
$$

where $\rho$ is the beam linear density, $\beta_{n}$ depends on the boundary conditions and ' $n$ ' denotes the index of the Euler-Bernoulli modes. This relation demonstrates that the natural frequencies $\omega_{n}$ of a beam depend on the stiffness and mass properties of beam, via $E, I, L, \rho$, and on the boundary conditions through $\beta_{n}$.

To evaluate the natural frequency of a beam with different boundary conditions, the following condition must be satisfied:

$$
\left|K_{T}-\omega^{2} M\right|=0
$$


Here, the bars denote the determinant, $M$ is the system mass matrix and $K_{T}$ is the total stiffness matrix given by the sum of the beam stiffness $K$, and stiffness due to penalty function $K_{P}$ as

$$
K_{T}=K+K_{P}
$$

To start, the determinant is calculated by considering the number of $A A F$ terms, to be $N=$ 5. The stiffness and mass matrices of an unconstrained beam are then given by

$$
K=\frac{E I}{L^{3}}\left[\begin{array}{ccccc}
0 & 0 & 0 & 0 & 0 \\
0 & 0 & 0 & 0 & 0 \\
0 & 0 & 4 & 0 & 0 \\
0 & 0 & 0 & \pi^{4} / 2 & 0 \\
0 & 0 & 0 & 0 & 8 \pi^{4}
\end{array}\right]
$$

and

$$
M=\rho L\left[\begin{array}{ccccc}
1 & 1 / 2 & 1 / 3 & 0 & 0 \\
1 / 2 & 1 / 3 & 1 / 4 & -2 / \pi^{2} & 0 \\
1 / 3 & 1 / 4 & 1 / 5 & -2 / \pi^{2} & 1 / 2 \pi^{2} \\
0 & -2 / \pi^{2} & -2 / \pi^{2} & 1 / 2 & 0 \\
0 & 0 & 1 / 2 \pi^{2} & 0 & 1 / 2
\end{array}\right]
$$

With the use of alternative admissible functions, changes in boundary conditions do not alter the stiffness and mass matrices given in Eq.(4.5) and Eq.(4.6). This stands in contrast to the traditional implementation of assumed modes approaches where the form of the assumed mode is typically changed with a change in the boundary conditions, hence changing the system mass and stiffness matrices. With the $A A F$ approach, boundary conditions are implemented via penalty functions, without altering the stiffness and mass matrix of the system. Estimation of appropriate penalty function for different boundary conditions with $A A F$ approach is explained in the following sections.

In this study, the properties of the beam are considered unaltered. Using Euler-Bernoulli relation for natural frequencies $\omega_{n}$, the value of $\beta_{n}, n=1 . . \mathrm{N}$. for different boundary conditions is estimated and considered for the following sections. It is important to note that ' $N$ ' is the number of alternative admissible function terms used to obtain the mass, stiffness, and penalty stiffness 
matrices of the system and ' $n$ ' is the index for the number of modes in $\beta_{n}$ as in Euler-Bernoulli theorem.

\subsubsection{Penalty Function for Clamped-Free $(\mathrm{CF})$ Boundary Condition}

To calculate the value of the penalty $(k)$ for Clamped-Free $(C F)$ boundary condition, the penalty matrix of $C F$ using number of $A A F$ terms $(N=5)$ is given by

$$
K_{P, C F}=\left[\begin{array}{ccccc}
k_{0} & 0 & 0 & k_{0} & k_{0} \\
0 & k_{r 0} & 0 & 0 & 0 \\
0 & 0 & 0 & 0 & 0 \\
k_{0} & 0 & 0 & k_{0} & k_{0} \\
k_{0} & 0 & 0 & k_{0} & k_{0}
\end{array}\right]
$$

Using the assumptions described in section 4.2 and substituting Eq. (4.1) in Eq. (4.7), the penalty matrix of $C F$ can be written as

$$
K_{P, C F}=\frac{E I}{L^{3}}\left[\begin{array}{lllll}
k & 0 & 0 & k & k \\
0 & k & 0 & 0 & 0 \\
0 & 0 & 0 & 0 & 0 \\
k & 0 & 0 & k & k \\
k & 0 & 0 & k & k
\end{array}\right]
$$

To calculate the value of penalty $(k)$ for Clamped-Free $(C F)$ boundary condition, the stiffness matrix of the unconstrained beam Eq.(4.5), the stiffness matrix of the $C F$ boundary condition Eq.(4.8), the Euler-Bernoulli relation for natural frequency Eq.(4.2) and the mass matrix of the unconstrained beam Eq.(4.6) considering number of $A A F$ terms $(N=5)$ are substituted in frequency equation of the beam Eq.(4.3) to give 


$$
\begin{aligned}
& \mid \frac{E I}{L^{3}}\left[\begin{array}{ccccc}
0 & 0 & 0 & 0 & 0 \\
0 & 0 & 0 & 0 & 0 \\
0 & 0 & 4 & 0 & 0 \\
0 & 0 & 0 & \pi^{4} / 2 & 0 \\
0 & 0 & 0 & 0 & 8 \pi^{4}
\end{array}\right]+\frac{E I}{L^{3}}\left[\begin{array}{ccccc}
k & 0 & 0 & k & k \\
0 & k & 0 & 0 & 0 \\
0 & 0 & 0 & 0 & 0 \\
k & 0 & 0 & k & k \\
k & 0 & 0 & k & k
\end{array}\right] \\
& -\left(\beta_{n} L\right)^{4} \frac{E I}{\rho L^{4}} \rho L\left[\begin{array}{ccccc}
1 & 1 / 2 & 1 / 3 & 0 & 0 \\
1 / 2 & 1 / 3 & 1 / 4 & -2 / \pi^{2} & 0 \\
1 / 3 & 1 / 4 & 1 / 5 & -2 / \pi^{2} & 1 / 2 \pi^{2} \\
0 & -2 / \pi^{2} & -2 / \pi^{2} & 1 / 2 & 0 \\
0 & 0 & 1 / 2 \pi^{2} & 0 & 1 / 2
\end{array}\right] \mid=0
\end{aligned}
$$

After simplifying the matrices, Eq. (4.9) leads to

$$
\begin{aligned}
& \mid \begin{array}{ccc}
k-\left(\beta_{n} L\right)^{4} & -\left(\beta_{n} L\right)^{4} / 2 & -\left(\beta_{n} L\right)^{4} / 3 \\
-\left(\beta_{n} L\right)^{4} / 2 & k-\left(\left(\beta_{n} L\right)^{4} / 3\right) & -\left(\beta_{n} L\right)^{4} / 4 \\
-\left(\beta_{n} L\right)^{4} / 3 & -\left(\beta_{n} L\right)^{4} / 4 & 4-\left(\left(\beta_{n} L\right)^{4} / 5\right) \\
k & 2\left(\beta_{n} L\right)^{4} / \pi^{2} & 2\left(\beta_{n} L\right)^{4} / \pi^{2} \\
k & 0 & -\left(\beta_{n} L\right)^{4} / 2 \pi^{2}
\end{array} \\
& k \\
& 2\left(\beta_{n} L\right)^{4} / \pi^{2} \\
& 2\left(\beta_{n} L\right)^{4} / \pi^{2} \\
& \left(\pi^{4} / 2\right)+k-\left(\left(\beta_{n} L\right)^{4} / 2\right) \\
& \begin{array}{c}
k \\
0 \\
-\left(\beta_{n} L\right)^{4} / 2 \pi^{2} \\
k \\
8 \pi^{4}+k-\left(\left(\beta_{n} L\right)^{4} / 2\right)
\end{array} \mid=0
\end{aligned}
$$

It is noticed that the size of the determinant is $5 \mathrm{X} 5$ since $N=5$, this indicates that the size of the determinant directly depends on the number of $A A F$ terms used. Here $N=5$ is chosen to obtain a minimum set of $A A F$ that includes both the lower order polynomials and trigonometric functions.

On setting the determinant equal to zero, Eq. (4.10) yields a quadratic polynomial equation in terms of $\left(\beta_{n} L\right)$ and value of the penalty $(k)$. Here ' $n$ ' indicates the mode index in the EulerBernoulli beam and is considered from $n=1$ to 12 . In the present study, first six natural frequencies are of interest. From a preliminary study conducted to estimate natural frequencies using the proposed approach, at least the first 12 natural frequencies need to be considered to obtain a reasonable percentage error in estimating the frequency. 
For a $C F$ boundary condition, the $\left(\beta_{n} L\right)$ value is calculated by Euler-Bernoulli theorem using $\frac{(2 n-1) \pi}{2}$ and is substituted in the quadratic polynomial. After calculating and substituting the $\left(\beta_{n} L\right)$ value for various $n$, the value of the penalty $(k)$ can be found by equating the quadratic polynomial to zero. The obtained quadratic polynomial yields two roots of $k$ as $k_{1}$ and $k_{2}$ corresponding to each value of $\left(\beta_{n} L\right)$ and $n$. Table 4-1 tabulates the values of $k_{1}$ and $k_{2}$ corresponding to each value of $\left(\beta_{n} L\right)$.

Table 4-1: $k_{1}$ and $k_{2}$ Values for $C F$ Boundary Condition

\begin{tabular}{|c|c|r|r|}
\hline $\boldsymbol{n}$ & $\left.\boldsymbol{( \beta}_{\boldsymbol{n}} \boldsymbol{L}\right)$ & \multicolumn{1}{c|}{$\boldsymbol{k}_{\boldsymbol{1}}$} & \multicolumn{1}{c|}{$\boldsymbol{k}_{\boldsymbol{2}}$} \\
\hline 1 & 1.8751 & $3,584.16$ & 1.10 \\
\hline 2 & 4.6941 & $3,180.51$ & -0.16 \\
\hline 3 & 7.8548 & 780.64 & -2.94 \\
\hline 4 & 10.9955 & $1,115.92$ & -23.22 \\
\hline 5 & 14.1372 & $6,652.03$ & 4.07 \\
\hline 6 & 17.2788 & $15,683.08$ & 20.21 \\
\hline 7 & 20.4204 & $31,117.24$ & 46.83 \\
\hline 8 & 23.5619 & $55,548.74$ & 88.73 \\
\hline 9 & 26.7035 & $91,964.20$ & 151.11 \\
\hline 10 & 29.8451 & $143,766.41$ & 239.81 \\
\hline 11 & 32.9867 & $214,781.84$ & 361.40 \\
\hline 12 & 36.1283 & $309,261.34$ & 523.14 \\
\hline
\end{tabular}

Considering the highest positive value of $k$ from Table 4-1, the relation for $k_{0}$ and $k_{r 0}$ yields

$$
k_{r 0}=k_{0}=\frac{E I}{L^{3}} k=\frac{E I}{L^{3}} \times 3,09,261.34
$$

Note that the magnitude of $k_{r 0}$ value is taken to be equal to the magnitude of $k_{0}$ for simplicity. However, the units of $k_{r 0}$ should be treated as ' $N-m / r a d$ ' and units of $k_{0}$ as ' $N / m$ '. Eq. (4.11) can be expressed in a conventional way in terms of $10^{\text {th }}$ to the power of index as given by 


$$
k_{0}=k_{r 0}=\frac{E I}{L^{3}} \times 10^{5.49}
$$

Eq. (4.12) shows that the value of penalty functions required to enforce the Clamped-Free $(C F)$ boundary conditions is directly proportional to the Young's modulus $(E)$ of the beam material, second moment of area of the cross section $(I)$ and is inversely proportional the length $(L)$ of the beam used. The value of the 10 to the power depends on the highest positive value of $k$. It can be varied depending on the mode of interest. A similar approach is carried out for simply-supported boundary condition and is outlined as follows.

\subsubsection{Penalty Function for Simply-Supported (SS) Boundary Condition}

For Simply-Supported (SS) boundary conditions a similar approach is carried out and the penalty stiffness matrix of a $S S$ boundary condition is given by,

$$
K_{P, S S}=\left[\begin{array}{ccccc}
2 k_{0} & k_{0} & k_{0} & 0 & 2 k_{0} \\
k_{0} & k_{0} & k_{0} & -k_{0} & k_{0} \\
k_{0} & k_{0} & k_{0} & -k_{0} & k_{0} \\
0 & -k_{0} & -k_{0} & 2 k_{0} & 0 \\
2 k_{0} & k_{0} & k_{0} & 0 & 2 k_{0}
\end{array}\right]
$$

Utilizing the assumptions described in section 4.2 and substituting Eq.(4.1) in Eq. (4.13), we obtain

$$
K_{P, S S}=\frac{E I}{L^{3}}\left[\begin{array}{ccccc}
2 k & k & k & 0 & 2 k \\
k & k & k & -k & k \\
k & k & k & -k & k \\
0 & -k & -k & 2 k & 0 \\
2 k & k & k & 0 & 2 k
\end{array}\right]
$$

The number of $A A F$ terms $(\mathrm{N}=5)$ is considered to obtain the stiffness, mass, and penalty stiffness matrices. The penalty value $(k)$ for $S S$ boundary condition requires only a change in the penalty stiffness matrix and remaining procedure is unaltered. Upon substituting the Eqs. (4.4),(4.5),(4.6),(4.2) and (4.14) into Eq. (4.3) we obtain 


$$
\begin{aligned}
& \mid \frac{E I}{L^{3}}\left[\begin{array}{ccccc}
0 & 0 & 0 & 0 & 0 \\
0 & 0 & 0 & 0 & 0 \\
0 & 0 & 4 & 0 & 0 \\
0 & 0 & 0 & \pi^{4} / 2 & 0 \\
0 & 0 & 0 & 0 & 8 \pi^{4}
\end{array}\right]+\frac{E I}{L^{3}}\left[\begin{array}{ccccc}
2 k & k & k & 0 & 2 k \\
k & k & k & -k & k \\
k & k & k & -k & k \\
0 & -k & -k & 2 k & 0 \\
2 k & k & k & 0 & 2 k
\end{array}\right] \\
& -\left(\beta_{n} L\right)^{4} \frac{E I}{\rho L^{4}} \rho L\left[\begin{array}{ccccc}
1 & 1 / 2 & 1 / 3 & 0 & 0 \\
1 / 2 & 1 / 3 & 1 / 4 & -2 / \pi^{2} & 0 \\
1 / 3 & 1 / 4 & 1 / 5 & -2 / \pi^{2} & 1 / 2 \pi^{2} \\
0 & -2 / \pi^{2} & -2 / \pi^{2} & 1 / 2 & 0 \\
0 & 0 & 1 / 2 \pi^{2} & 0 & 1 / 2
\end{array}\right] \mid=0
\end{aligned}
$$

which simplifies to give

$$
\begin{aligned}
& \mid \begin{array}{ccc}
2 k-\left(\beta_{n} L\right)^{4} & k-\left(\beta_{n} L\right)^{4} / 2 & k-\left(\beta_{n} L\right)^{4} / 3 \\
k-\left(\beta_{n} L\right)^{4} / 2 & k-B / 3 & k-B / 4 \\
k-B / 3 & k-B / 4 & 4+k-(B / 5) \\
0 & -k+2 B / \pi^{2} & -k+2 B / \pi^{2} \\
2 k & k & k-B / 2 \pi^{2}
\end{array} \\
& \begin{array}{cc}
0 & 2 k \\
-k+2 B / \pi^{2} & k \\
-k+2 B / \pi^{2} & k-B / 2 \pi^{2} \\
\left(\pi^{4} / 2\right)+2 k-(B / 2) & 0 \\
0 & 8 \pi^{4}+2 k-(B / 2)
\end{array} \mid=0
\end{aligned}
$$

On setting the determinant equal to zero, Eq. (4.16) leads to a quadratic polynomial equation, in terms of $k$ and with $\left(\beta_{n} L\right)$ as a parameter. Here ' $n$ ' indicates the number of modes in Euler-Bernoulli theorem and is considered from $n=1$ to 12 . In this thesis, first six natural frequencies are studied and according to Meirovitch [1] the number of modes ' $n$ ' should be twice the number of frequencies of interest, hence $n=12$. The $\left(\beta_{n} L\right)$ for $S S$ boundary condition is calculated used Euler-Bernoulli equation $n \pi$. The quadratic polynomial equation can be solved for $k$ upon substituting the calculated values of $\left(\beta_{n} L\right)$ for the Simply-Supported (SS) boundary conditions from $n=1$ to 12 and equating the polynomial to zero giving the two roots i.e., $k_{l}$ and $k_{2}$. The values of $k_{1}$ and $k_{2}$ corresponding to each value of $\left(\beta_{n} L\right)$ and $n$ when substituted in the quadratic polynomial equation, are tabulated in Table 4-2. 
Table 4-2: $k_{1}$ and $k_{2}$ Values for $S S$ Boundary Condition

\begin{tabular}{|c|c|r|r|}
\hline $\boldsymbol{n}$ & $\boldsymbol{( \boldsymbol { \beta } _ { \boldsymbol { n } } \boldsymbol { L } )}$ & \multicolumn{1}{c|}{$\boldsymbol{k}_{\boldsymbol{1}}$} & \multicolumn{1}{c|}{$\boldsymbol{k}_{\boldsymbol{2}}$} \\
\hline 1 & 3.1416 & $4,256.72$ & 16.76 \\
\hline 2 & 6.2832 & 735.16 & 121.47 \\
\hline 3 & 9.4248 & $1,729.44$ & 84.02 \\
\hline 4 & 12.5664 & $1,054.42$ & -313.63 \\
\hline 5 & 15.7080 & $2,971.82$ & $1,466.91$ \\
\hline 6 & 18.8496 & $6,437.75$ & $4,048.09$ \\
\hline 7 & 21.9911 & $12,139.69$ & $8,186.79$ \\
\hline 8 & 25.1327 & $20,884.05$ & $14,500.75$ \\
\hline 9 & 28.2743 & $33,599.91$ & $23,669.35$ \\
\hline 10 & 31.4159 & $51,339.79$ & $36,454.51$ \\
\hline 11 & 34.5575 & $75,279.92$ & $53,705.18$ \\
\hline 12 & 37.6991 & $106,720.25$ & $76,358.67$ \\
\hline
\end{tabular}

Considering the highest positive value of $k$ from Table 4-2, the relation for $k_{0}$ and $k_{r 0}$ yields follow,

$$
k_{r 0}=k_{0}=\frac{E I}{L^{3}} k=\frac{E I}{L^{3}} \times 1,06,720.25
$$

Eq. (4.17) can be expressed in a conventional way in terms of $10^{\text {th }}$ to the power of index as

$$
k_{0}=k_{r 0}=\frac{E I}{L^{3}} \times 10^{5.03}
$$

Eq. (4.18) shows that the value of penalty functions required to enforce the Simply-Supported (SS) boundary condition is directly proportional to the Young's modulus $(E)$ of the beam material, second moment of area of the cross section $(I)$ and inversely proportional the length $(L)$ of the beam used. This relation obtained holds good for all types of material and geometrical parameters of a beam with Simply-Supported (SS) boundary condition.

\subsubsection{Penalty Function for Clamped-Clamped (CC) Boundary Condition}

The penalty function for Clamped-Clamped $(C C)$ boundary conditions using number of $A A F$ terms as $N=5$ is given by 


$$
K_{P, C C}=\left[\begin{array}{ccccc}
2 k_{0} & k_{0} & k_{0} & 0 & 2 k_{0} \\
k_{0} & 3 k_{0} & 3 k_{0} & -k_{0} & k_{0} \\
k_{0} & 3 k_{0} & 5 k_{0} & -k_{0} & k_{0} \\
0 & -k_{0} & -k_{0} & 2 k_{0} & 0 \\
2 k_{0} & k_{0} & k_{0} & 0 & 2 k_{0}
\end{array}\right]
$$

Substituting Eq. (4.5) in Eq.(4.19), we obtain

$$
K_{P, C C}=\frac{E I}{L^{3}}\left[\begin{array}{ccccc}
2 k & k & k & 0 & 2 k \\
k & 3 k & 3 k & -k & k \\
k & 3 k & 5 k & -k & k \\
0 & -k & -k & 2 k & 0 \\
2 k & k & k & 0 & 2 k
\end{array}\right]
$$

Upon substituting the Eqs. (4.4),(4.5),(4.6),(4.2) and (4.20) into Eq. (4.3) we obtain

$$
\begin{aligned}
& \mid \frac{E I}{L^{3}}\left[\begin{array}{ccccc}
0 & 0 & 0 & 0 & 0 \\
0 & 0 & 0 & 0 & 0 \\
0 & 0 & 4 & 0 & 0 \\
0 & 0 & 0 & \pi^{4} / 2 & 0 \\
0 & 0 & 0 & 0 & 8 \pi^{4}
\end{array}\right]+\frac{E I}{L^{3}}\left[\begin{array}{ccccc}
2 k & k & k & 0 & 2 k \\
k & 3 k & 3 k & -k & k \\
k & 3 k & 5 k & -k & k \\
0 & -k & -k & 2 k & 0 \\
2 k & k & k & 0 & 2 k
\end{array}\right] \\
& -\left(\beta_{n} L\right)^{4} \frac{E I}{\rho L^{4}} \rho L\left[\begin{array}{ccccc}
1 & 1 / 2 & 1 / 3 & 0 & 0 \\
1 / 2 & 1 / 3 & 1 / 4 & -2 / \pi^{2} & 0 \\
1 / 3 & 1 / 4 & 1 / 5 & -2 / \pi^{2} & 1 / 2 \pi^{2} \\
0 & -2 / \pi^{2} & -2 / \pi^{2} & 1 / 2 & 0 \\
0 & 0 & 1 / 2 \pi^{2} & 0 & 1 / 2
\end{array}\right] \mid
\end{aligned}
$$

which simplifies to

$$
\left|\begin{array}{cccc}
2 k-B & k-B / 2 & k-B / 3 \\
k-B / 2 & 3 k-(B / 3) & 3 k-B / 4 & \\
k-B / 3 & 3 k-B / 4 & 4+5 k-(B / 5) \\
0 & -k+2 B / \pi^{2} & -K+2 B / \pi^{2} \\
2 k & k & k-B / 2 \pi^{2} & \\
& 0 & 2 k \\
& -k+2 B / \pi^{2} & k \\
& -k+2 B / \pi^{2} & k-B / 2 \pi^{2} \\
& \left(\pi^{4} / 2\right)+2 k-(B / 2) & 0 \\
0 & 8 \pi^{4}+2 k-(B / 2)
\end{array}\right|=0
$$

On setting the determinant equal to zero, Eq. (4.22) leads to quartic polynomial equation i.e., a polynomial of $4^{\text {th }}$ order, with $k$ and $\left(\beta_{n} L\right)$ as unknow parameters. For a $C C$ boundary condition, 
the $\left(\beta_{n} L\right)$ value is calculated using Euler-Bernoulli theorem as $\frac{(2 n+1) \pi}{2}$. Substituting the values of $\left(\beta_{n} L\right)$ for Clamped-Clamped $(C C)$ boundary conditions from $n=1$ to 12 in the $4^{\text {th }}$ order polynomial and equating it to zero, gives four roots of $k_{\text {as }} k_{1}, k_{2}, k_{3}$, and $k_{4}$. The values of $k_{1}, k_{2}, k_{3}$ and $k_{4}$ corresponding to each value of $\left(\beta_{n} L\right)$ and $n$ are tabulated in Table 4-3.

Table 4-3: $k_{1}, k_{2}, k_{3}$ and $k_{4}$ Values for $C C$ Boundary Condition

\begin{tabular}{|c|c|r|r|r|r|}
\hline $\boldsymbol{n}$ & $\left.\boldsymbol{(} \boldsymbol{\beta}_{\boldsymbol{n}} \boldsymbol{L}\right)$ & \multicolumn{1}{c|}{$\boldsymbol{k}_{\boldsymbol{1}}$} & \multicolumn{1}{c|}{$\boldsymbol{k}_{\boldsymbol{2}}$} & \multicolumn{1}{c|}{$\boldsymbol{k}_{\boldsymbol{3}}$} & \multicolumn{1}{c|}{$\boldsymbol{k}_{\boldsymbol{4}}$} \\
\hline 1 & 4.7300 & 4867.43 & 100.79 & 0.00 & -4.66 \\
\hline 2 & 7.8532 & 933.06 & 433.37 & -1.80 & -7.97 \\
\hline 3 & 10.9956 & $3,660.81$ & $2,263.10$ & 4.74 & -3.38 \\
\hline 4 & 14.1372 & $10,049.38$ & $6,506.22$ & 19.89 & 2.27 \\
\hline 5 & 17.2788 & $22,457.97$ & $14,741.18$ & 49.28 & 12.71 \\
\hline 6 & 20.4204 & $43,835.17$ & $28,926.51$ & 99.91 & 30.58 \\
\hline 7 & 23.5619 & $77,717.93$ & $51,409.64$ & 180.15 & 58.85 \\
\hline 8 & 26.7035 & $128,236.31$ & $84,931.22$ & 299.79 & 100.99 \\
\hline 9 & 29.8451 & $200,107.16$ & $1,32,621.19$ & 469.99 & 160.94 \\
\hline 10 & 32.9867 & $298,637.88$ & $1,98,001.28$ & 703.33 & 243.12 \\
\hline 11 & 36.1283 & $429,725.66$ & $2,84,984.59$ & $1,013.77$ & 352.45 \\
\hline 12 & 39.2699 & $599,857.31$ & $3,97,875.47$ & $1,416.67$ & 494.35 \\
\hline
\end{tabular}

Considering the highest positive value of $k$ from Table 4-3, the relation for $k_{0}$ and $k_{r 0}$ yields

$$
k_{r 0}=k_{0}=\frac{E I}{L^{3}} k=\frac{E I}{L^{3}} \times 5,99,857.31
$$

Eq. (4.1) can be expressed in a conventional way in terms of $10^{\text {th }}$ to the power of index as given by

$$
k_{0}=k_{r 0}=\frac{E I}{L^{3}} \times 10^{5.78}
$$

Eq. (4.24) shows that the value of penalty functions required to enforce the Clamped-Clamped $(C C)$ boundary condition is directly proportional to the Young's modulus $(E)$ of the beam material, second moment of area of the cross section $(I)$ and inversely proportional the length $(L)$ of the 
beam used. This relation obtained holds for all types of material and geometrical parameters of a beam with Clamped-Clamped $(C C)$ boundary condition. It is noted that the required value of $\mathrm{k}$ for the Clamped-Clamped boundary condition is higher than for simply-supported or clamped-free boundary conditions. This is consistent with the idea that clamping both sides of the beam make the overall system stiffer (the natural frequencies are higher). Hence, higher values of penalty functions are required to artificially synthesize this condition.

\subsubsection{Effect of Number of Terms $(N)$ on Penalty Value}

The procedure is extended to study the effect of number of alternative admissible terms $(N)$ on the estimated penalty value. The number of alternative admissible terms $(N)$ determines the size of the stiffness, mass, and penalty matrices. The expression of penalty for $C F$ boundary condition is $k_{0}=$ $k_{r 0}=\frac{E I}{L^{3}} \times 10^{5.49}$ and is obtained in the section 4.4.2 where $N$ is considered as five. In this study, the number of $A A F$ terms $(N)$ are varied from $N=6$ to 50 . Here, the index of number of modes $(n)$ of interest for this study is unaltered. From preliminary simulations to obtain minimum percentage error in estimating the natural frequencies, the index of number of modes $(n)$ is considered to be twice the index of the frequency of interest, therefore $n=12$.

For various $N$ values, the corresponding determinant is evaluated. It is noticed that the number of $A A F$ terms $(N)$ does not affect the order of the polynomial. For a $C F$ boundary condition, the order of the polynomial is quadratic. Substituting $\left(\beta_{n} L\right)$ values in the quadratic polynomial and equating it to zero gives roots of $k$ as $k_{l}$ and $k_{2}$. Table 4-4 tabulates the obtained highest positive value of $k$ and the estimated penalty values in terms of $10^{\text {th }}$ to the power of index for various $N$ values. 
Table 4-4: Value of Penalty $(k)$ for Various $N$ Terms

\begin{tabular}{|c|c|}
\hline $\boldsymbol{N}$ & $\begin{array}{c}\text { Value of the Penalty } \\
(\boldsymbol{k})\end{array}$ \\
\hline 5 & $309,261=1 \times 10^{5.49}$ \\
\hline 6 & $230,004=1 \times 10^{5.36}$ \\
\hline 7 & $181,546=1 \times 10^{5.26}$ \\
\hline 8 & $148,762=1 \times 10^{5.17}$ \\
\hline 9 & $124,602=1 \times 10^{5.10}$ \\
\hline 10 & $104,762=1 \times 10^{5.02}$ \\
\hline 11 & $124,903=1 \times 10^{5.10}$ \\
\hline 12 & $168,357=1 \times 10^{5.23}$ \\
\hline 13 & $217,137=1 \times 10^{5.34}$ \\
\hline 15 & $329,258=1 \times 10^{5.52}$ \\
\hline 25 & $2,017,833=1 \times 10^{6.30}$ \\
\hline 30 & $N a N$ \\
\hline 50 & $N a N$ \\
\hline
\end{tabular}

It is observed in Table 4-4 that as the value of $N$ increases, the penalty value decreases up to $N=$ 10 and increases again up to $N=25$. Further, the value of penalty for $N=30$ and above results in 'NaN' (Not a Number) in Matlab, indicating non-determinate number. It could potentially be interpreted as a very high penalty value approaching infinity (values start to diverge). It is observed that the penalty value in terms of $10^{\text {th }}$ to the power of index are in the same range of $1 \mathrm{X} 10^{5.10}$ to $1 \mathrm{X} 10^{5.52}$ until $N=15$ and tend to increase afterwards. Table 4-4 demonstrates that there is not much change in the values of the penalty functions for values of $N$ between 5 and 15 . Since the first three 
terms in the set of $A A F$ are polynomials with the rest of the terms as trigonometric functions, the smallest number of $N$ for which the $A A F$ approach can reasonably be used as assumed modes is $N$ $=5$. Even for $N=25$, the change in penalty is less than an order of magnitude. Hence for the rest of the analysis, $N=5$ will be used.

Similar observations are seen for $S S$ and $C C$ boundary conditions. The order of the polynomial is quadratic and quartic polynomial equations, respectively. On calculating and substituting corresponding $\beta_{n} L$ values depending on the boundary condition, it was observed the change in $N$ does not affect the estimated penalty values. Hence for the rest of the study, the number of $A A F$ terms used to calculate the penalty value is $N=5$. The following section outlines the generalized methodology used to obtain the penalty value of various boundary conditions.

\subsection{Methodology}

The penalty matrices for the three boundary conditions, namely Clamped-Free $(C F)$, SimplySupported (SS) and Clamped-Clamped (CC) are defined by introducing virtual linear $\left(k_{0}\right)$ and rotational $\left(k_{r 0}\right)$ springs in place of the actual boundary conditions, as explained in section 4.2. The present methodology provides flexibility and provides easy method to compute penalty values with changes in boundary conditions. The procedure essentially requires only to change the penalty stiffness matrix depending on the boundary condition. The procedure for obtaining the penalty function $\left[K_{P}\right]$ and utilizing the obtained relation to calculate the natural frequencies and modeshapes are indicated in the form of a flow chart as shown in Figure 4-1.In the present, it is necessary to note that ' $N$ ' is the number of AAF terms used to obtain the mass, stiffness, and penalty stiffness matrices of the system and ' $n$ ' is the index for the number of modes in $\beta_{n}$ as in Euler-Bernoulli theorem. 


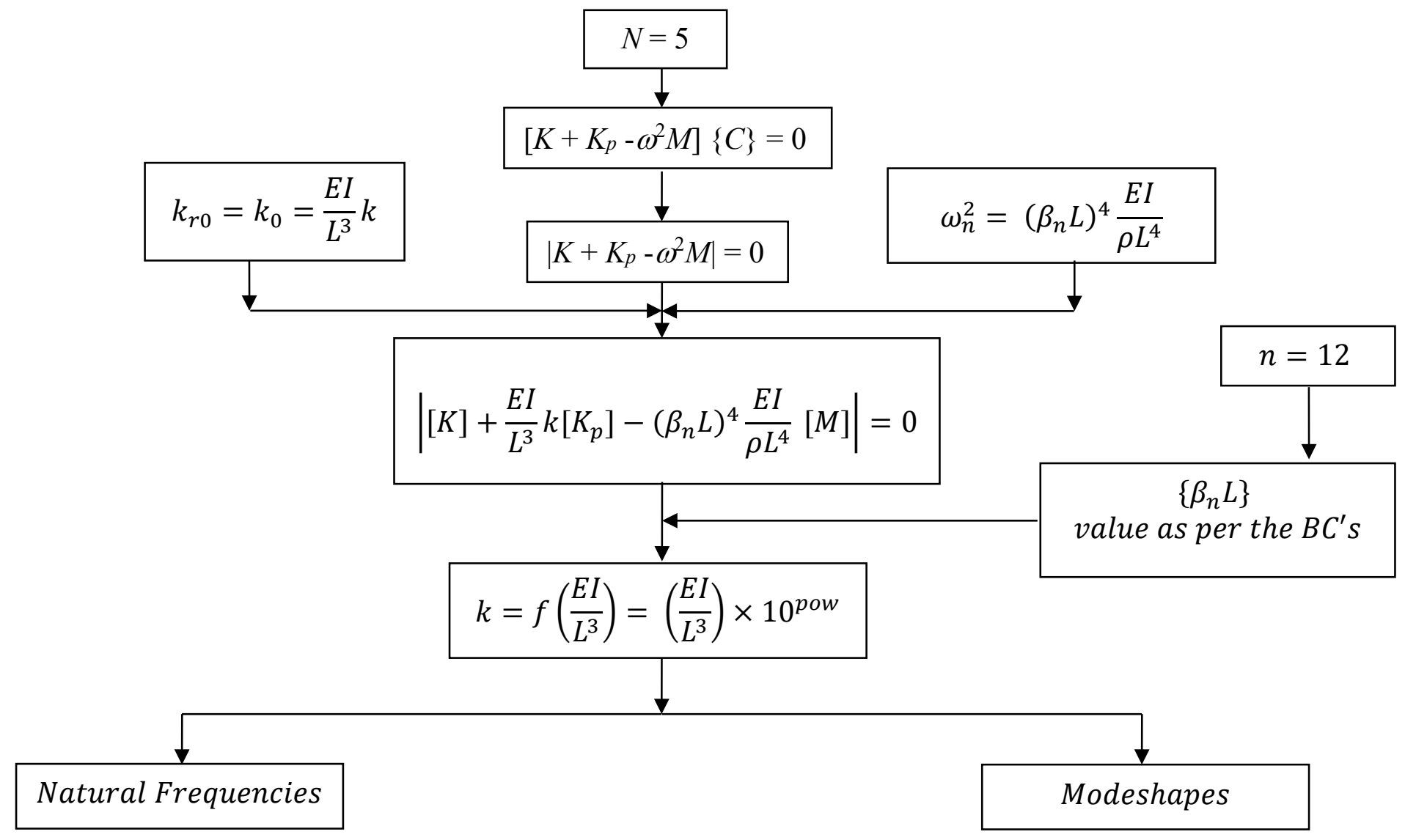

Figure 4-1: Flow-Chart for Selection of Penalty Functions

To calculate the natural frequencies and modeshapes of a structure using $A A F$ with penalties the following procedure is proposed. In chapter 3 , it is observed that the penalty value depends on the boundary condition and as well as the underlying beam properties. The observed relationship between the penalty value and the beam properties is incorporated using the assumption for virtual linear and rotational springs via Eq.(4.1). The natural frequency of the beam is expressed in terms of different boundary conditions using the Euler-Bernoulli relationship Eq.(4.2). Here, the number of $A A F$ terms considered in the formation of the stiffness, mass, and penalty stiffness matrixes is chosen as $N=5$. The equation of motion of the beam holds the information about the stiffness and mass matrix of the unconstrained beam. The different boundary conditions are incorporated using respective stiffness penalty matrices. By substituting all the assumptions, matrices and setting the determinant of the equation of motion equal to zero, a polynomial is obtained. For a $C F$ and $S S$ boundary condition, the obtained order of polynomial is 
quadratic. However, for a $C C$ boundary condition the order of polynomial is quartic. The $\left(\beta_{n} L\right)$ value using Euler-Bernoulli relation depends on the different boundary condition and is calculated for $n$ different values. On substituting the $\left(\beta_{n} L\right)$ values in the characteristic polynomial leads to set of $k$ roots depending on the type of order of polynomial. Considering the highest value of the root represented in form of $10^{\text {th }}$ to the power of index, the value of penalty for different boundary conditions is obtained as a function of the beam properties. Using the obtained relation for penalty value and adopting the $A A F$ method, the number of natural frequencies and modeshapes of interest are then calculated.

\subsection{Validation of Selection of Penalty Functions}

Mathematically derived models can represent the physical phenomenon when those mathematical models are applicable and validated. Obtained mathematical expressions for penalty functions for all three boundary conditions are validated considering a beam with some geometrical parameters and material characteristics as explained in section 4.4.1.

\subsubsection{Geometrical Parameters and Material Characteristics}

An Euler- Bernoulli beam, as shown in Figure 3-1, is considered for validation. Table 4-5 shows the material properties of the beam considered.

Table 4-5: Material Properties of Aluminium

\begin{tabular}{|l|c|}
\hline \multicolumn{1}{|c|}{ Parameter } & Values \\
\hline Young's Modulus (E) & $69.79 \mathrm{GPa}$ \\
\hline Mass Density $(\rho)$ & $2,600 \mathrm{~kg} / \mathrm{m}^{3}$ \\
\hline
\end{tabular}

Validation of results for Clamped-Free $(C F)$ boundary conditions is examined in the following section. In the following tables from Table 4-7 to Table 4-35 the value of penalty is considered as 
$k_{0}=1 \mathrm{X} 10^{10}$ as stated in previous literature [2], [3], [11] for the purpose of validation of the present approach.

\subsubsection{Validation for Clamped-Free $(C F)$ Boundary Condition}

The relation for the value of penalty functions required to enforce Clamped-Free $(C F)$ boundary conditions is discussed in section 4.2.1. The obtained expression indicates a direct relation between Young's modulus of the beam material, second moment of area of the cross section and the length of the beam used.

Three different lengths of beam are chosen as $0.5 \mathrm{~m}, 1 \mathrm{~m}$, and $1.5 \mathrm{~m}$, with three different thicknesses of $3 \mathrm{~mm}, 4 \mathrm{~mm}$, and $5 \mathrm{~mm}$ for validation. The specific values of calculated penalty functions using Eq.(4.12) for the material and geometrical parameters considered are tabulated in Table 4-6.

Table 4-6: Calculated Values of Penalty Function $C F$ Boundary Condition

\begin{tabular}{|c|c|c|c|}
\hline \multicolumn{4}{|c|}{$\left[k_{0}=k_{r 0}=\frac{E I}{L^{3}} \times 10^{5.49}\right]$} \\
\hline $\begin{array}{l}\text { Length }(\mathrm{m}) / \\
\text { thickness }(\mathrm{m})\end{array}$ & 0.5 & 1 & 1.5 \\
\hline $3 \times 10^{-3}$ & $1 \times 10^{7.29}$ & $1 \times 10^{6.39}$ & $1 \times 10^{5.86}$ \\
\hline $4 \times 10^{-3}$ & $1 \times 10^{7.66}$ & $1 \times 10^{6.76}$ & $1 \times 10^{6.23}$ \\
\hline $5 \times 10^{-3}$ & $1 \times 10^{7.95}$ & $1 \times 10^{7.05}$ & $1 \times 10^{6.52}$ \\
\hline
\end{tabular}

It is noted that as the beam length increases, the calculated value required to enforce the boundary condition decreases and increases as the thickness of the beam increases. It can be observed that the values of penalty function chosen are specific to the selected beam properties. Using alternative admissible function with penalties, these values can directly be incorporated for enforcing the Clamped-Free $(C F)$ boundary conditions. 
The natural frequencies calculated using the present value of penalty functions is compared with the Euler-Bernoulli Beam and penalty value selected from the literature. Table 4-6 to Table 4-14 tabulate the comparison and validation of results for different dimensions shown in Table 4-5. Nine cases with different lengths and thicknesses are considered for the validation of results under these conditions. From Table 4-7 to Table 4-9, the thickness of the beam is kept constant at $3 \mathrm{~mm}$ and the length of the beam is varied from $0.5 \mathrm{~m}$ to $1.5 \mathrm{~m}$ with an increment of $0.5 \mathrm{~m}$. The value of penalty for combination of different lengths and thicknesses under each case are calculated using the established relationship. Table 4-7 tabulates the predicted natural frequency using the estimated value of penalty function of $k_{0}=1 \times 10^{7.29}$ for a beam length of $0.5 \mathrm{~m}$ and $3 \mathrm{~mm}$ thickness. Similarly, Table 4-8 and Table 4-9 accommodate the predicted natural frequency corresponding to estimated penalty functions of $k_{0}=1 \mathrm{X} 10^{6.39}$ and $k_{0}=1 \mathrm{X} 10^{5.86}$ for $1 \mathrm{~m}$ and $1.5 \mathrm{~m}$ beam length respectively. The $k_{0}=1 \mathrm{X} 10^{10}$ value is from the literature and the $k_{0}=1 \mathrm{X} 10^{7.29}$ value is from the proposed methodology.

Table 4-7: Natural Frequencies of $C F$ Beam with $L=0.5 \mathrm{~m}$ and $h=3 \mathrm{~mm}$.

\begin{tabular}{|c|c|c|c|r|r|r|r|}
\hline \multicolumn{2}{|c|}{$N=12$} & \multicolumn{3}{|c|}{$\boldsymbol{k}_{\mathbf{0}}=\mathbf{1 X 1 0}^{\mathbf{1 0}}$} & \multicolumn{3}{c|}{$\boldsymbol{k}_{\mathbf{0}}=\mathbf{1 X 1 0}^{\mathbf{7 . 2 9}}$} \\
\hline Mode & $\begin{array}{c}\mathbf{E B B} \\
\mathbf{( H z )}\end{array}$ & $\begin{array}{c}\mathbf{A A F} \\
\mathbf{( H z )}\end{array}$ & $\begin{array}{c}\text { \% } \\
\text { Error }\end{array}$ & $\begin{array}{c}\text { Dev. } \\
(\mathbf{H z})\end{array}$ & $\begin{array}{c}\mathbf{A A F} \\
\mathbf{( H z )}\end{array}$ & $\begin{array}{c}\text { \% } \\
\text { Error }\end{array}$ & $\begin{array}{c}\text { Dev. } \\
(\mathbf{H z})\end{array}$ \\
\hline $1^{\text {st }}$ & 10.04 & 10.04 & 0.00 & 0.00 & 10.04 & 0.00 & 0.00 \\
\hline $2^{\text {nd }}$ & 62.94 & 62.96 & 0.04 & 0.02 & 62.95 & 0.02 & 0.01 \\
\hline $3^{\text {rd }}$ & 176.23 & 176.40 & 0.10 & 0.17 & 176.33 & 0.06 & 0.10 \\
\hline $4^{\text {th }}$ & 345.35 & 346.04 & 0.20 & 0.70 & 345.77 & 0.12 & 0.43 \\
\hline $5^{\text {th }}$ & 570.88 & 572.61 & 0.30 & 1.73 & 571.89 & 0.18 & 1.01 \\
\hline $6^{\text {th }}$ & 852.80 & 857.55 & 0.56 & 4.76 & 856.00 & 0.38 & 3.20 \\
\hline
\end{tabular}


Table 4-8: Natural Frequencies of $C F$ Beam with $L=1.0 \mathrm{~m}$ and $h=3 \mathrm{~mm}$

\begin{tabular}{|c|c|r|r|r|r|r|r|}
\hline \multicolumn{2}{|c|}{$N=\mathbf{1 2}$} & \multicolumn{3}{c|}{$\boldsymbol{k}_{\mathbf{0}}=\mathbf{1 X 1 0}$} & \multicolumn{3}{c|}{$\boldsymbol{k}_{\mathbf{0}}=\mathbf{1 X 1 0}^{\mathbf{6 3} 9}$} \\
\hline Mode & $\begin{array}{c}\text { EBB } \\
(\mathbf{H z})\end{array}$ & $\begin{array}{c}\text { AAF } \\
(\mathbf{H z})\end{array}$ & $\begin{array}{c}\text { \% } \\
\text { Error }\end{array}$ & $\begin{array}{c}\text { Dev. } \\
(\mathbf{H z})\end{array}$ & $\begin{array}{c}\text { AAF } \\
(\mathbf{H z})\end{array}$ & $\begin{array}{c}\text { \% } \\
\text { Error }\end{array}$ & $\begin{array}{c}\text { Dev. } \\
(\mathbf{H z})\end{array}$ \\
\hline $1^{\text {st }}$ & 2.51 & 2.51 & 0.00 & 0.00 & 2.51 & 0.00 & 0.00 \\
\hline $2^{\text {nd }}$ & 15.73 & 15.74 & 0.04 & 0.01 & 15.74 & 0.02 & 0.00 \\
\hline $3^{\text {rd }}$ & 44.06 & 44.10 & 0.10 & 0.04 & 44.08 & 0.06 & 0.02 \\
\hline $4^{\text {th }}$ & 86.34 & 86.51 & 0.20 & 0.17 & 86.44 & 0.12 & 0.11 \\
\hline $5^{\text {th }}$ & 142.72 & 143.15 & 0.30 & 0.43 & 142.97 & 0.18 & 0.25 \\
\hline $6^{\text {th }}$ & 213.20 & 214.39 & 0.56 & 1.19 & 214.00 & 0.38 & 0.80 \\
\hline
\end{tabular}

Table 4-9: Natural Frequencies of $C F$ Beam with $L=1.5 \mathrm{~m}$ and $h=3 \mathrm{~mm}$

\begin{tabular}{|c|c|r|r|r|r|r|r|}
\hline \multicolumn{2}{|c|}{$N=12$} & \multicolumn{3}{c|}{$k_{\mathbf{0}}=\mathbf{1 X 1 0}^{\mathbf{1 0}}$} & \multicolumn{3}{c|}{$\boldsymbol{k}_{\mathbf{0}}=\mathbf{1 X 1 0}^{5.86}$} \\
\hline Mode & $\begin{array}{c}\text { EBB } \\
(\mathbf{H z})\end{array}$ & $\begin{array}{c}\text { AAF } \\
(\mathbf{H z})\end{array}$ & $\begin{array}{c}\text { \% } \\
\text { Error }\end{array}$ & $\begin{array}{c}\text { Dev. } \\
(\mathbf{H z})\end{array}$ & $\begin{array}{c}\text { AAF } \\
(\mathbf{H z})\end{array}$ & $\begin{array}{c}\text { \% } \\
\text { Error }\end{array}$ & $\begin{array}{c}\text { Dev. } \\
(\mathbf{H z})\end{array}$ \\
\hline $1^{\text {st }}$ & 1.12 & 1.12 & 0.00 & 0.00 & 1.12 & 0.00 & 0.00 \\
\hline $2^{\text {nd }}$ & 6.99 & 7.00 & 0.04 & 0.00 & 6.99 & 0.02 & 0.00 \\
\hline $3^{\text {rd }}$ & 19.58 & 19.60 & 0.10 & 0.02 & 19.59 & 0.06 & 0.01 \\
\hline $4^{\text {th }}$ & 38.37 & 38.45 & 0.20 & 0.08 & 38.42 & 0.12 & 0.05 \\
\hline $5^{\text {th }}$ & 63.43 & 63.62 & 0.30 & 0.19 & 63.54 & 0.18 & 0.11 \\
\hline $6^{\text {th }}$ & 94.76 & 95.28 & 0.56 & 0.53 & 95.11 & 0.38 & 0.36 \\
\hline
\end{tabular}

For Table 4-10 to Table 4-12, the length of the beam is varied from $0.5 \mathrm{~m}$ to $1.5 \mathrm{~m}$ and the thickness of the beam is kept constant at $4 \mathrm{~mm}$. The frequencies corresponding to values of penalties for $k_{0}=$ $1 \mathrm{X} 10^{7.66}, k_{0}=1 \times 10^{6.76}$ and $k_{0}=1 \mathrm{X} 10^{6.23}$ for a beam length of $0.5 \mathrm{~m}, 1 \mathrm{~m}$ and $1.5 \mathrm{~m}$ are tabulated. 
Table 4-10: Natural Frequencies of $C F$ Beam with $L=0.5 \mathrm{~m}$ and $h=4 \mathrm{~mm}$

\begin{tabular}{|c|c|r|r|r|r|r|r|}
\hline \multicolumn{2}{|c|}{$N=\mathbf{1 2}$} & \multicolumn{3}{c|}{$\boldsymbol{k}_{\mathbf{0}}=\mathbf{1 X 1 0}$} & \multicolumn{3}{c|}{$\boldsymbol{k}_{\mathbf{0}}=\mathbf{1 X 1 0}$} \\
\hline Mode & $\begin{array}{c}\text { EBB } \\
(\mathbf{H z})\end{array}$ & $\begin{array}{c}\text { AAF } \\
(\mathbf{H z})\end{array}$ & $\begin{array}{c}\text { \% } \\
\text { Error }\end{array}$ & $\begin{array}{c}\text { Dev. } \\
(\mathbf{H z})\end{array}$ & $\begin{array}{c}\text { AAF } \\
(\mathbf{H z})\end{array}$ & $\begin{array}{c}\text { \% } \\
\text { Error }\end{array}$ & $\begin{array}{c}\text { Dev. } \\
(\mathbf{H z})\end{array}$ \\
\hline $1^{\text {st }}$ & 13.39 & 13.39 & 0.00 & 0.00 & 13.39 & 0.00 & 0.00 \\
\hline $2^{\text {nd }}$ & 83.92 & 83.95 & 0.04 & 0.03 & 83.94 & 0.02 & 0.02 \\
\hline $3^{\text {rd }}$ & 234.98 & 235.20 & 0.10 & 0.23 & 235.11 & 0.06 & 0.13 \\
\hline $4^{\text {th }}$ & 460.46 & 461.39 & 0.20 & 0.93 & 461.03 & 0.12 & 0.57 \\
\hline $5^{\text {th }}$ & 761.17 & 763.48 & 0.30 & 2.31 & 762.52 & 0.18 & 1.35 \\
\hline $6^{\text {th }}$ & 1137.06 & 1143.40 & 0.56 & 6.34 & 1141.33 & 0.38 & 4.26 \\
\hline
\end{tabular}

Table 4-11: Natural Frequencies of $C F$ Beam with $L=1.0 \mathrm{~m}$ and $h=4 \mathrm{~mm}$

\begin{tabular}{|c|c|r|r|r|r|r|r|}
\hline \multicolumn{2}{|c|}{$N=\mathbf{1 2}$} & \multicolumn{3}{c|}{$\boldsymbol{k}_{\mathbf{0}}=\mathbf{1 X 1 0}^{\mathbf{1 0}}$} & \multicolumn{3}{c|}{$\boldsymbol{k}_{\mathbf{0}}=\mathbf{1 X 1 0}^{\mathbf{6 . 7 6}}$} \\
\hline Mode & $\begin{array}{c}\text { EBB } \\
(\mathbf{H z})\end{array}$ & $\begin{array}{c}\text { AAF } \\
(\mathbf{H z})\end{array}$ & $\begin{array}{c}\text { \% } \\
\text { Error }\end{array}$ & $\begin{array}{c}\text { Dev. } \\
(\mathbf{H z})\end{array}$ & $\begin{array}{c}\text { AAF } \\
(\mathbf{H z})\end{array}$ & $\begin{array}{c}\text { \% } \\
\text { Error }\end{array}$ & $\begin{array}{c}\text { Dev. } \\
(\mathbf{H z})\end{array}$ \\
\hline $1^{\text {st }}$ & 3.35 & 3.35 & 0.00 & 0.00 & 3.35 & 0.00 & 0.00 \\
\hline $2^{\text {nd }}$ & 20.98 & 20.99 & 0.04 & 0.01 & 20.98 & 0.02 & 0.00 \\
\hline $3^{\text {rd }}$ & 58.74 & 58.80 & 0.10 & 0.06 & 58.78 & 0.06 & 0.03 \\
\hline $4^{\text {th }}$ & 115.12 & 115.35 & 0.20 & 0.23 & 115.26 & 0.12 & 0.14 \\
\hline $5^{\text {th }}$ & 190.29 & 190.87 & 0.30 & 0.58 & 190.63 & 0.18 & 0.34 \\
\hline $6^{\text {th }}$ & 284.27 & 285.85 & 0.56 & 1.59 & 285.33 & 0.38 & 1.07 \\
\hline
\end{tabular}


Table 4-12: Natural Frequencies of $C F$ Beam with $L=1.5 \mathrm{~m}$ and $h=4 \mathrm{~mm}$

\begin{tabular}{|c|r|r|r|r|r|r|r|}
\hline \multicolumn{2}{|c|}{$N=\mathbf{1 2}$} & \multicolumn{3}{c|}{$\boldsymbol{k}_{\mathbf{0}}=\mathbf{1 X 1 0}$} & \multicolumn{3}{c|}{$\boldsymbol{k}_{\mathbf{0}}=\mathbf{1 X 1 0}$} \\
\hline Mode & $\begin{array}{c}\text { EBB } \\
(\mathbf{H z})\end{array}$ & $\begin{array}{c}\text { AAF } \\
(\mathbf{H z})\end{array}$ & $\begin{array}{c}\text { \% } \\
\text { Error }\end{array}$ & $\begin{array}{c}\text { Dev. } \\
(\mathbf{H z})\end{array}$ & $\begin{array}{c}\text { AAF } \\
(\mathbf{H z})\end{array}$ & $\begin{array}{c}\text { \% } \\
\text { Error }\end{array}$ & $\begin{array}{c}\text { Dev. } \\
(\mathbf{H z})\end{array}$ \\
\hline $1^{\text {st }}$ & 1.49 & 1.49 & 0.00 & 0.00 & 1.49 & 0.00 & 0.00 \\
\hline $2^{\text {nd }}$ & 9.32 & 9.33 & 0.04 & 0.00 & 9.33 & 0.02 & 0.00 \\
\hline $3^{\text {rd }}$ & 26.11 & 26.13 & 0.10 & 0.03 & 26.12 & 0.06 & 0.01 \\
\hline $4^{\text {th }}$ & 51.16 & 51.27 & 0.20 & 0.10 & 51.23 & 0.12 & 0.06 \\
\hline $5^{\text {th }}$ & 84.57 & 84.83 & 0.30 & 0.26 & 84.72 & 0.18 & 0.15 \\
\hline $6^{\text {th }}$ & 126.34 & 127.05 & 0.56 & 0.71 & 126.81 & 0.38 & 0.47 \\
\hline
\end{tabular}

Similarly, from Table 4-13 to Table 4-15, the thickness of the beam is kept constant at $5 \mathrm{~mm}$ and the corresponding values of natural frequencies for different lengths are tabulated.

Table 4-13: Natural Frequencies of $C F$ Beam with $L=0.5 \mathrm{~m}$ and $h=5 \mathrm{~mm}$

\begin{tabular}{|c|c|c|c|c|c|c|c|}
\hline \multicolumn{2}{|c|}{$N=\mathbf{1 2}$} & \multicolumn{3}{c|}{$\boldsymbol{k}_{\mathbf{0}}=\mathbf{1 X 1 0}^{\mathbf{1 0}}$} & \multicolumn{3}{c|}{$\boldsymbol{k}_{\mathbf{0}}=\mathbf{1 X 1 0}^{\mathbf{7 . 9 5}}$} \\
\hline Mode & $\begin{array}{c}\text { EBB } \\
(\mathbf{H z})\end{array}$ & $\begin{array}{c}\text { AAF } \\
(\mathbf{H z})\end{array}$ & $\begin{array}{c}\text { \% } \\
\text { Error }\end{array}$ & $\begin{array}{c}\text { Dev. } \\
(\mathbf{H z})\end{array}$ & $\begin{array}{c}\text { AAF } \\
(\mathbf{H z})\end{array}$ & $\begin{array}{c}\text { \% } \\
\text { Error }\end{array}$ & $\begin{array}{c}\text { Dev. } \\
(\mathbf{H z})\end{array}$ \\
\hline $1^{\text {st }}$ & 16.74 & 16.74 & 0.00 & 0.00 & 16.74 & 0.00 & 0.00 \\
\hline $2^{\text {nd }}$ & 104.90 & 104.94 & 0.04 & 0.04 & 104.92 & 0.02 & 0.02 \\
\hline $3^{\text {rd }}$ & 293.72 & 294.00 & 0.10 & 0.28 & 293.88 & 0.06 & 0.16 \\
\hline $4^{\text {th }}$ & 575.58 & 576.73 & 0.20 & 1.16 & 576.29 & 0.12 & 0.71 \\
\hline $5^{\text {th }}$ & 951.47 & 954.35 & 0.30 & 2.88 & 953.15 & 0.18 & 1.68 \\
\hline $6^{\text {th }}$ & 1421.33 & 1429.24 & 0.56 & 7.91 & 1426.66 & 0.38 & 5.33 \\
\hline
\end{tabular}


Table 4-14: Natural Frequencies of $C F$ Beam with $L=1.0 \mathrm{~m}$ and $h=5 \mathrm{~mm}$

\begin{tabular}{|c|c|r|r|r|r|r|r|}
\hline \multicolumn{2}{|c|}{$N=\mathbf{1 2}$} & \multicolumn{3}{c|}{$\boldsymbol{k}_{\mathbf{0}}=\mathbf{1 X 1 0}^{\mathbf{1 0}}$} & \multicolumn{3}{c|}{$\boldsymbol{k}_{\mathbf{0}}=\mathbf{1 X 1 0}^{\mathbf{7 . 0 5}}$} \\
\hline Mode & $\begin{array}{c}\text { EBB } \\
(\mathbf{H z})\end{array}$ & $\begin{array}{c}\text { AAF } \\
(\mathbf{H z})\end{array}$ & $\begin{array}{c}\text { \% } \\
\text { Error }\end{array}$ & $\begin{array}{c}\text { Dev. } \\
(\mathbf{H z})\end{array}$ & $\begin{array}{c}\text { AAF } \\
(\mathbf{H z})\end{array}$ & $\begin{array}{c}\text { \% } \\
\text { Error }\end{array}$ & $\begin{array}{c}\text { Dev. } \\
(\mathbf{H z})\end{array}$ \\
\hline $1^{\text {st }}$ & 4.18 & 4.18 & 0.00 & 0.00 & 4.18 & 0.00 & 0.00 \\
\hline $2^{\text {nd }}$ & 26.22 & 26.23 & 0.04 & 0.01 & 26.23 & 0.02 & 0.01 \\
\hline $3^{\text {rd }}$ & 73.43 & 73.50 & 0.10 & 0.07 & 73.47 & 0.06 & 0.04 \\
\hline $4^{\text {th }}$ & 143.89 & 144.18 & 0.20 & 0.29 & 144.07 & 0.12 & 0.18 \\
\hline $5^{\text {th }}$ & 237.87 & 238.59 & 0.30 & 0.72 & 238.29 & 0.18 & 0.42 \\
\hline $6^{\text {th }}$ & 355.33 & 357.31 & 0.56 & 1.98 & 356.67 & 0.38 & 1.33 \\
\hline
\end{tabular}

Table 4-15: Natural Frequencies of $C F$ Beam with $L=1.5 \mathrm{~m}$ and $h=5 \mathrm{~mm}$

\begin{tabular}{|c|c|r|r|r|r|r|r|}
\hline \multicolumn{2}{|c|}{$N=\mathbf{1 2}$} & \multicolumn{3}{c|}{$\boldsymbol{k}_{\mathbf{0}}=\mathbf{1 X 1 0}^{\mathbf{1 0}}$} & \multicolumn{3}{c|}{$\boldsymbol{k}_{\mathbf{0}}=\mathbf{1 X 1 0}^{\mathbf{7 . 5 2}}$} \\
\hline Mode & $\begin{array}{c}\text { EBB } \\
(\mathbf{H z})\end{array}$ & $\begin{array}{c}\text { AAF } \\
(\mathbf{H z})\end{array}$ & $\begin{array}{c}\text { \% } \\
\text { Error }\end{array}$ & $\begin{array}{c}\text { Dev. } \\
(\mathbf{H z})\end{array}$ & $\begin{array}{c}\text { AAF } \\
(\mathbf{H z})\end{array}$ & $\begin{array}{c}\text { \% } \\
\text { Error }\end{array}$ & $\begin{array}{c}\text { Dev. } \\
(\mathbf{H z})\end{array}$ \\
\hline $1^{\text {st }}$ & 1.86 & 1.86 & 0.00 & 0.00 & 1.86 & 0.00 & 0.00 \\
\hline $2^{\text {nd }}$ & 11.66 & 11.66 & 0.04 & 0.00 & 11.66 & 0.02 & 0.00 \\
\hline $3^{\text {rd }}$ & 32.64 & 32.67 & 0.10 & 0.03 & 32.65 & 0.06 & 0.02 \\
\hline $4^{\text {th }}$ & 63.95 & 64.08 & 0.20 & 0.13 & 64.03 & 0.12 & 0.08 \\
\hline $5^{\text {th }}$ & 105.72 & 106.04 & 0.30 & 0.32 & 105.91 & 0.18 & 0.19 \\
\hline $6^{\text {th }}$ & 157.93 & 158.81 & 0.56 & 0.88 & 158.52 & 0.38 & 0.59 \\
\hline
\end{tabular}

It is noticed from Table 4-6 to Table 4-14 that the percentage of error in predicting the natural frequency obtained using the penalty function values estimated with the proposed procedure is lower as compared to when using the penalty values adopted directly from the literature. The validation is further carried out for simply-supported and clamped-clamped condition for the effectiveness of the method. 


\subsubsection{Validation for Simply-Supported (SS) Boundary Condition}

The relation for the value of penalty functions required to enforce Simply-Supported (SS) boundary conditions discussed in section 4.2.2, is considered for validation. In this case, as in the prior case, the obtained expression indicates a direct relation between Young's modulus of the beam material, second moment of area of the cross section and the length of the beam used.

Three different lengths of beam $0.5 \mathrm{~m}, 1 \mathrm{~m}$, and $1.5 \mathrm{~m}$, with three different thicknesses of $3 \mathrm{~mm}$, $4 \mathrm{~mm}$ and $5 \mathrm{~mm}$ are considered for validation purposes. The specific values of calculated penalty functions using Eq.(4.18) for the material and geometrical parameters considered are tabulated in Table 4-16.

Table 4-16: Calculated Values of Penalty Function for SS Boundary Conditions

\begin{tabular}{|c|c|c|c|}
\multicolumn{4}{|c}{$\left[k_{0}=k_{r 0}=\frac{E I}{L^{3}} \times 10^{5.03}\right]$} \\
\hline $\begin{array}{c}\text { Length }(\mathrm{m}) / \\
\text { thickness }(\mathrm{m})\end{array}$ & 0.5 & 1 & 1.5 \\
\hline $3 \times 10^{-3}$ & $1 \times 10^{6.83}$ & $1 \times 10^{5.92}$ & $1 \times 10^{5.39}$ \\
\hline $4 \times 10^{-3}$ & $1 \times 10^{7.20}$ & $1 \times 10^{6.30}$ & $1 \times 10^{5.77}$ \\
\hline $5 \times 10^{-3}$ & $1 \times 10^{7.49}$ & $1 \times 10^{6.59}$ & $1 \times 10^{6.06}$ \\
\hline
\end{tabular}

It is noticed that as the beam length increases, the calculated value required to enforce the boundary condition decreases and increases as the thickness of the beam increases. Using alternative admissible function with penalties, these values can directly be incorporated for enforcing the Simply-Supported (SS) boundary conditions.

Similar to the Clamped-Free $(C F)$ boundary condition, nine cases with different lengths and thicknesses are considered for the validation of results under $S S$ conditions. The natural frequencies are calculated using the proposed method of estimation of value of penalty functions. The natural frequencies obtained using the penalty values adopted from literature and penalty 
values from the proposed method are compared. Table 4-17 to Table 4-25 tabulate the comparison and validation of results for calculation of natural frequencies for beams of different dimensions as indicated in Table 4-16. Table 4-17 tabulates the predicted natural frequencies using the estimated value of penalty function of $k_{0}=1 \times 10^{6.83}$ for a beam length of $0.5 \mathrm{~m}$ and $3 \mathrm{~mm}$ thickness. In the following tables from Table 4-17 to Table 4-25 the value of penalty is considered as $k_{0}=1 \times 10^{10}$ as stated in previous literature [2], [3], [11] for the purpose of validation of the present approach.

Table 4-17: Natural Frequencies of SS Beam with $L=0.5 \mathrm{~m}$ and $h=3 \mathrm{~mm}$

\begin{tabular}{|c|c|c|c|c|c|c|c|}
\hline \multicolumn{2}{|c|}{$N=12$} & \multicolumn{3}{|c|}{$k_{0}=1 \times 10^{10}$} & \multicolumn{3}{|c|}{$k_{0}=1 \mathrm{X} 10^{6.83}$} \\
\hline Mode & $\begin{array}{l}\text { EBB } \\
(\mathrm{Hz})\end{array}$ & $\begin{array}{l}\text { AAF } \\
\text { (Hz) }\end{array}$ & $\begin{array}{c}\% \\
\text { Error }\end{array}$ & $\begin{array}{l}\text { Dev. } \\
\text { (Hz) }\end{array}$ & $\begin{array}{l}\text { AAF } \\
\text { (Hz) }\end{array}$ & $\begin{array}{c}\% \\
\text { Error }\end{array}$ & $\begin{array}{l}\text { Dev. } \\
\text { (Hz) }\end{array}$ \\
\hline $1^{\text {st }}$ & 10.04 & 10.04 & 0.00 & 0.00 & 10.04 & 0.00 & 0.00 \\
\hline $2^{\text {nd }}$ & 62.94 & 62.96 & 0.04 & 0.02 & 62.93 & 0.01 & 0.01 \\
\hline $3^{\text {rd }}$ & 176.23 & 176.40 & 0.10 & 0.17 & 176.20 & 0.02 & 0.04 \\
\hline $4^{\text {th }}$ & 345.35 & 346.04 & 0.20 & 0.70 & 345.26 & 0.03 & 0.09 \\
\hline $5^{\text {th }}$ & 570.88 & 572.61 & 0.30 & 1.73 & 570.49 & 0.07 & 0.39 \\
\hline $6^{\text {th }}$ & 852.80 & 857.55 & 0.56 & 4.76 & 852.91 & 0.01 & 0.12 \\
\hline
\end{tabular}


Table 4-18: Natural Frequencies of SS Beam with $L=1.0 \mathrm{~m}$ and $h=3 \mathrm{~mm}$

\begin{tabular}{|c|c|r|r|r|r|r|r|}
\hline \multicolumn{2}{|c|}{$N=\mathbf{1 2}$} & \multicolumn{3}{c|}{$\boldsymbol{k}_{\mathbf{0}}=\mathbf{1 X 1 0}^{\mathbf{1 0}}$} & \multicolumn{3}{c|}{$\boldsymbol{k}_{\mathbf{0}}=\mathbf{1 X 1 0}$} \\
\hline Mode & $\begin{array}{c}\text { EBB } \\
(\mathbf{H z})\end{array}$ & $\begin{array}{c}\text { AAF } \\
(\mathbf{H z})\end{array}$ & $\begin{array}{c}\text { \% } \\
\text { Error }\end{array}$ & $\begin{array}{c}\text { Dev. } \\
(\mathbf{H z})\end{array}$ & $\begin{array}{c}\text { AAF } \\
(\mathbf{H z})\end{array}$ & $\begin{array}{c}\text { \% } \\
\text { Error }\end{array}$ & $\begin{array}{c}\text { Dev. } \\
(\mathbf{H z})\end{array}$ \\
\hline $1^{\text {st }}$ & 2.51 & 2.51 & 0.00 & 0.00 & 2.51 & 0.00 & 0.00 \\
\hline $2^{\text {nd }}$ & 15.73 & 15.74 & 0.04 & 0.01 & 15.73 & 0.01 & 0.00 \\
\hline $3^{\text {rd }}$ & 44.06 & 44.10 & 0.10 & 0.04 & 44.05 & 0.02 & -0.01 \\
\hline $4^{\text {th }}$ & 86.34 & 86.51 & 0.20 & 0.17 & 86.31 & 0.03 & -0.02 \\
\hline $5^{\text {th }}$ & 142.72 & 143.15 & 0.30 & 0.43 & 142.62 & 0.07 & -0.10 \\
\hline $6^{\text {th }}$ & 213.20 & 214.39 & 0.56 & 1.19 & 213.23 & 0.01 & 0.03 \\
\hline
\end{tabular}

Table 4-19: Natural Frequencies of SS Beam with $L=1.5 \mathrm{~m}$ and $h=3 \mathrm{~mm}$

\begin{tabular}{|c|c|r|r|r|r|r|r|}
\hline \multicolumn{2}{|c|}{$N=\mathbf{1 2}$} & \multicolumn{3}{c|}{$\boldsymbol{k}_{\mathbf{0}}=\mathbf{1 X 1 0}$} & \multicolumn{3}{c|}{$\boldsymbol{k}_{\mathbf{0}}=\mathbf{1 X 1 0}$} \\
\hline Mode & $\begin{array}{c}\text { EBB } \\
(\mathbf{H z})\end{array}$ & $\begin{array}{c}\text { AAF } \\
(\mathbf{H z})\end{array}$ & $\begin{array}{c}\text { \% } \\
\text { Error }\end{array}$ & $\begin{array}{c}\text { Dev. } \\
(\mathbf{H z})\end{array}$ & $\begin{array}{c}\text { AAF } \\
(\mathbf{H z})\end{array}$ & $\begin{array}{c}\text { \% } \\
\text { Error }\end{array}$ & $\begin{array}{c}\text { Dev. } \\
(\mathbf{H z})\end{array}$ \\
\hline $1^{\text {st }}$ & 1.12 & 1.12 & 0.00 & 0.00 & 1.12 & 0.00 & 0.00 \\
\hline $2^{\text {nd }}$ & 6.99 & 7.00 & 0.04 & 0.00 & 6.99 & 0.01 & 0.00 \\
\hline $3^{\text {rd }}$ & 19.58 & 19.60 & 0.10 & 0.02 & 19.58 & 0.02 & 0.00 \\
\hline $4^{\text {th }}$ & 38.37 & 38.45 & 0.20 & 0.08 & 38.36 & 0.03 & -0.01 \\
\hline $5^{\text {th }}$ & 63.43 & 63.62 & 0.30 & 0.19 & 63.39 & 0.07 & -0.04 \\
\hline $6^{\text {th }}$ & 94.76 & 95.28 & 0.56 & 0.53 & 94.77 & 0.01 & 0.01 \\
\hline
\end{tabular}

Similarly, Table 4-19 shows the predicted natural frequencies corresponding to estimated penalty functions of $k_{0}=1 \mathrm{X} 10^{5.92}$ and $k_{0}=1 \mathrm{X} 10^{5.39}$ for $1 \mathrm{~m}$ and $1.5 \mathrm{~m}$ beam length respectively. From Table 4-20 to Table 4-22, the length of the beam is varied from $0.5 \mathrm{~m}$ to $1.5 \mathrm{~m}$ and the thickness of the beam is kept constant at $4 \mathrm{~mm}$. Using the methodology proposed above, the estimated values of penalties are $k_{0}=1 \times 10^{7.20}, k_{0}=1 \times 10^{6.30}$ and $k_{0}=1 \times 10^{5.77}$ for a beam length of $0.5 \mathrm{~m}, 1 \mathrm{~m}$ and $1.5 \mathrm{~m}$ respectively are tabulated. 
Table 4-20: Natural Frequencies of SS Beam with $L=0.5 \mathrm{~m}$ and $h=4 \mathrm{~mm}$

\begin{tabular}{|c|c|r|r|r|r|r|r|}
\hline \multicolumn{2}{|c|}{$N=\mathbf{1 2}$} & \multicolumn{3}{c|}{$\boldsymbol{k}_{\mathbf{0}}=\mathbf{1 X 1 0}^{\mathbf{1 0}}$} & \multicolumn{3}{c|}{$\boldsymbol{k}_{\mathbf{0}}=\mathbf{1 X 1 0}^{7.20}$} \\
\hline Mode & $\begin{array}{c}\text { EBB } \\
(\mathbf{H z})\end{array}$ & $\begin{array}{c}\text { AAF } \\
(\mathbf{H z})\end{array}$ & $\begin{array}{c}\text { \% } \\
\text { Error }\end{array}$ & $\begin{array}{c}\text { Dev. } \\
(\mathbf{H z})\end{array}$ & $\begin{array}{c}\text { AAF } \\
(\mathbf{H z})\end{array}$ & $\begin{array}{c}\text { \% } \\
\text { Error }\end{array}$ & $\begin{array}{c}\text { Dev. } \\
(\mathbf{H z})\end{array}$ \\
\hline $1^{\text {st }}$ & 13.39 & 13.39 & 0.00 & 0.00 & 13.39 & 0.00 & 0.00 \\
\hline $2^{\text {nd }}$ & 83.92 & 83.95 & 0.04 & 0.03 & 83.91 & 0.01 & -0.01 \\
\hline $3^{\text {rd }}$ & 234.98 & 235.20 & 0.10 & 0.23 & 234.93 & 0.02 & -0.05 \\
\hline $4^{\text {th }}$ & 460.46 & 461.39 & 0.20 & 0.93 & 460.34 & 0.03 & -0.12 \\
\hline $5^{\text {th }}$ & 761.17 & 763.48 & 0.30 & 2.31 & 760.66 & 0.07 & -0.52 \\
\hline $6^{\text {th }}$ & 1137.06 & 1143.40 & 0.56 & 6.34 & 1137.22 & 0.01 & 0.16 \\
\hline
\end{tabular}

Table 4-21: Natural Frequencies of $S S$ Beam with $L=1.0 \mathrm{~m}$ and $h=4 \mathrm{~mm}$

\begin{tabular}{|c|c|r|r|r|r|r|r|}
\hline \multicolumn{2}{|c|}{$N=\mathbf{1 2}$} & \multicolumn{3}{c|}{$\boldsymbol{k}_{\mathbf{0}}=\mathbf{1 X 1 0}^{\mathbf{1 0}}$} & \multicolumn{3}{c|}{$\boldsymbol{k}_{\mathbf{0}}=\mathbf{1 X 1 0}^{6.30}$} \\
\hline Mode & $\begin{array}{c}\text { EBB } \\
(\mathbf{H z})\end{array}$ & $\begin{array}{c}\text { AAF } \\
(\mathbf{H z})\end{array}$ & $\begin{array}{c}\text { \% } \\
\text { Error }\end{array}$ & $\begin{array}{c}\text { Dev. } \\
(\mathbf{H z})\end{array}$ & $\begin{array}{c}\text { AAF } \\
(\mathbf{H z})\end{array}$ & $\begin{array}{c}\text { \% } \\
\text { Error }\end{array}$ & $\begin{array}{c}\text { Dev. } \\
(\mathbf{H z})\end{array}$ \\
\hline $1^{\text {st }}$ & 3.35 & 3.35 & 0.00 & 0.00 & 3.35 & 0.00 & 0.00 \\
\hline $2^{\text {nd }}$ & 20.98 & 20.99 & 0.04 & 0.01 & 20.98 & 0.01 & 0.00 \\
\hline $3^{\text {rd }}$ & 58.74 & 58.80 & 0.10 & 0.06 & 58.73 & 0.02 & -0.01 \\
\hline $4^{\text {th }}$ & 115.12 & 115.35 & 0.20 & 0.23 & 115.09 & 0.03 & -0.03 \\
\hline $5^{\text {th }}$ & 190.29 & 190.87 & 0.30 & 0.58 & 190.16 & 0.07 & -0.13 \\
\hline $6^{\text {th }}$ & 284.27 & 285.85 & 0.56 & 1.59 & 284.30 & 0.01 & 0.04 \\
\hline
\end{tabular}


Table 4-22: Natural Frequencies of SS Beam with $L=1.5 \mathrm{~m}$ and $h=4 \mathrm{~mm}$

\begin{tabular}{|c|c|r|r|r|r|r|r|}
\hline \multicolumn{2}{|c|}{$N=\mathbf{1 2}$} & \multicolumn{3}{c|}{$\boldsymbol{k}_{\mathbf{0}}=\mathbf{1 X 1 0}$} & \multicolumn{3}{c|}{$\boldsymbol{k}_{\mathbf{0}}=\mathbf{1 X 1 0}$} \\
\hline Mode & $\begin{array}{c}\text { EBB } \\
(\mathbf{H z})\end{array}$ & $\begin{array}{c}\text { AAF } \\
(\mathbf{H z})\end{array}$ & $\begin{array}{c}\text { \% } \\
\text { Error }\end{array}$ & $\begin{array}{c}\text { Dev. } \\
(\mathbf{H z})\end{array}$ & $\begin{array}{c}\text { AAF } \\
(\mathbf{H z})\end{array}$ & $\begin{array}{c}\text { \% } \\
\text { Error }\end{array}$ & $\begin{array}{c}\text { Dev. } \\
(\mathbf{H z})\end{array}$ \\
\hline $1^{\text {st }}$ & 1.49 & 1.49 & 0.00 & 0.00 & 1.49 & 0.00 & 0.00 \\
\hline $2^{\text {nd }}$ & 9.32 & 9.33 & 0.04 & 0.00 & 9.32 & 0.01 & 0.00 \\
\hline $3^{\text {rd }}$ & 26.11 & 26.13 & 0.10 & 0.03 & 26.10 & 0.02 & -0.01 \\
\hline $4^{\text {th }}$ & 51.16 & 51.27 & 0.20 & 0.10 & 51.15 & 0.03 & -0.01 \\
\hline $5^{\text {th }}$ & 84.57 & 84.83 & 0.30 & 0.26 & 84.52 & 0.07 & -0.06 \\
\hline $6^{\text {th }}$ & 126.34 & 127.05 & 0.56 & 0.71 & 126.36 & 0.01 & 0.02 \\
\hline
\end{tabular}

Similarly, from Table 4-23 to Table 4-25 the thickness of the beam is kept constant at $5 \mathrm{~mm}$ and the corresponding values of natural frequencies for different lengths are tabulated.

Table 4-23: Natural Frequencies of SS Beam with $L=0.5 \mathrm{~m}$ and $h=5 \mathrm{~mm}$

\begin{tabular}{|c|c|c|c|c|c|c|c|}
\hline \multicolumn{2}{|c|}{$N=\mathbf{1 2}$} & \multicolumn{3}{c|}{$\boldsymbol{k}_{\mathbf{0}}=\mathbf{1 X 1 0}^{10}$} & \multicolumn{3}{c|}{$\boldsymbol{k}_{\mathbf{0}}=\mathbf{1 X 1 0}^{\mathbf{7 . 4 9}}$} \\
\hline Mode & $\begin{array}{c}\text { EBB } \\
(\mathbf{H z})\end{array}$ & $\begin{array}{c}\text { AAF } \\
(\mathbf{H z})\end{array}$ & $\begin{array}{c}\text { \% } \\
\text { Error }\end{array}$ & $\begin{array}{c}\text { Dev. } \\
(\mathbf{H z})\end{array}$ & $\begin{array}{c}\text { AAF } \\
(\mathbf{H z})\end{array}$ & $\begin{array}{c}\text { \% } \\
\text { Error }\end{array}$ & $\begin{array}{c}\text { Dev. } \\
(\mathbf{H z})\end{array}$ \\
\hline $1^{\text {st }}$ & 16.74 & 16.74 & 0.00 & 0.00 & 16.74 & 0.00 & 0.00 \\
\hline $2^{\text {nd }}$ & 104.90 & 104.94 & 0.04 & 0.04 & 104.89 & 0.01 & -0.01 \\
\hline $3^{\text {rd }}$ & 293.72 & 294.00 & 0.10 & 0.28 & 293.66 & 0.02 & -0.06 \\
\hline $4^{\text {th }}$ & 575.58 & 576.73 & 0.20 & 1.16 & 575.43 & 0.03 & -0.15 \\
\hline $5^{\text {th }}$ & 951.47 & 954.35 & 0.30 & 2.88 & 950.82 & 0.07 & -0.65 \\
\hline $6^{\text {th }}$ & 1421.33 & 1429.24 & 0.56 & 7.91 & 1421.52 & 0.01 & 0.19 \\
\hline
\end{tabular}


Table 4-24: Natural Frequencies of SS Beam with $L=1.0 \mathrm{~m}$ and $h=5 \mathrm{~mm}$

\begin{tabular}{|c|c|r|r|r|r|r|r|}
\hline \multicolumn{2}{|c|}{$N=\mathbf{1 2}$} & \multicolumn{3}{c|}{$\boldsymbol{k}_{\mathbf{0}}=\mathbf{1 X 1 0}^{10}$} & \multicolumn{3}{c|}{$\boldsymbol{k}_{\mathbf{0}}=\mathbf{1 X 1 0}$} \\
\hline Mode & $\begin{array}{c}\text { EBB } \\
(\mathbf{H z})\end{array}$ & $\begin{array}{c}\text { AAF } \\
(\mathbf{H z})\end{array}$ & $\begin{array}{c}\text { \% } \\
\text { Error }\end{array}$ & $\begin{array}{c}\text { Dev. } \\
(\mathbf{H z})\end{array}$ & $\begin{array}{c}\text { AAF } \\
(\mathbf{H z})\end{array}$ & $\begin{array}{c}\text { \% } \\
\text { Error }\end{array}$ & $\begin{array}{c}\text { Dev. } \\
(\mathbf{H z})\end{array}$ \\
\hline $1^{\text {st }}$ & 4.18 & 4.18 & 0.00 & 0.00 & 4.18 & 0.00 & 0.00 \\
\hline $2^{\text {nd }}$ & 26.22 & 26.23 & 0.04 & 0.01 & 26.22 & 0.01 & 0.00 \\
\hline $3^{\text {rd }}$ & 73.43 & 73.50 & 0.10 & 0.07 & 73.41 & 0.02 & -0.02 \\
\hline $4^{\text {th }}$ & 143.89 & 144.18 & 0.20 & 0.29 & 143.86 & 0.03 & -0.04 \\
\hline $5^{\text {th }}$ & 237.87 & 238.59 & 0.30 & 0.72 & 237.71 & 0.07 & -0.16 \\
\hline $6^{\text {th }}$ & 355.33 & 357.31 & 0.56 & 1.98 & 355.38 & 0.01 & 0.05 \\
\hline
\end{tabular}

Table 4-25: Natural Frequencies of SS Beam with $L=1.5 \mathrm{~m}$ and $h=5 \mathrm{~mm}$

\begin{tabular}{|c|c|r|r|r|r|r|r|}
\hline \multicolumn{2}{|c|}{$N=\mathbf{1 2}$} & \multicolumn{3}{c|}{$\boldsymbol{k}_{\mathbf{0}}=\mathbf{1 X 1 0}^{\mathbf{1 0}}$} & \multicolumn{3}{c|}{$\boldsymbol{K}_{\boldsymbol{0}}=\mathbf{1 X 1 0}^{6.06}$} \\
\hline Mode & $\begin{array}{c}\text { EBB } \\
(\mathbf{H z})\end{array}$ & $\begin{array}{c}\text { AAF } \\
(\mathbf{H z})\end{array}$ & $\begin{array}{c}\text { \% } \\
\text { Error }\end{array}$ & $\begin{array}{c}\text { Dev. } \\
(\mathbf{H z})\end{array}$ & $\begin{array}{c}\text { AAF } \\
(\mathbf{H z})\end{array}$ & $\begin{array}{c}\text { \% } \\
\text { Error }\end{array}$ & $\begin{array}{c}\text { Dev. } \\
(\mathbf{H z})\end{array}$ \\
\hline $1^{\text {st }}$ & 1.86 & 1.86 & 0.00 & 0.00 & 1.86 & 0.00 & 0.00 \\
\hline $2^{\text {nd }}$ & 11.66 & 11.66 & 0.04 & 0.00 & 11.65 & 0.01 & 0.00 \\
\hline $3^{\text {rd }}$ & 32.64 & 32.67 & 0.10 & 0.03 & 32.63 & 0.02 & -0.01 \\
\hline $4^{\text {th }}$ & 63.95 & 64.08 & 0.20 & 0.13 & 63.94 & 0.03 & -0.02 \\
\hline $5^{\text {th }}$ & 105.72 & 106.04 & 0.30 & 0.32 & 105.65 & 0.07 & -0.07 \\
\hline $6^{\text {th }}$ & 157.93 & 158.81 & 0.56 & 0.88 & 157.95 & 0.01 & 0.02 \\
\hline
\end{tabular}

It is noticed from Table 4-17 to Table 4-25 that the percentage error in predicting the natural frequency obtained using the penalty function obtained from the proposed methodology is lower compared to the values adopted directly from literature.

\subsubsection{Validation for Clamped-Clamped (CC) Boundary Condition}

The proposed approach for validation of Clamped-Clamped $(C C)$ boundary is presented in this section. A similar approach was considered as for the clamped-free and simply-supported cases. 
Three different beam lengths were considered. The length of the beam is increased, keeping the thickness constant. The specific values of calculated penalty functions using Eq.(4.24) for the material and geometrical parameters considered are tabulated in Table 4-26.

Table 4-26: Calculated Values of Penalty Function for $C C$ Boundary Conditions

\begin{tabular}{|c|c|c|c|}
\hline \multicolumn{4}{|c|}{$\left[k_{0}=k_{r 0}=\frac{E I}{L^{3}} \times 10^{5.78}\right]$} \\
\hline $\begin{array}{l}\text { Length }(\mathrm{m}) / \\
\text { thickness }(\mathrm{m})\end{array}$ & 0.5 & 1 & 1.5 \\
\hline $3 \times 10^{-3}$ & $1 \times 10^{7.58}$ & $1 \times 10^{6.67}$ & $1 \times 10^{6.14}$ \\
\hline $4 \times 10^{-3}$ & $1 \times 10^{7.95}$ & $1 \times 10^{7.05}$ & $1 \times 10^{6.52}$ \\
\hline $5 \times 10^{-3}$ & $1 \times 10^{8.24}$ & $1 \times 10^{7.34}$ & $1 \times 10^{6.81}$ \\
\hline
\end{tabular}

It is noticed that as the beam length increases, the calculated value required to enforce the boundary condition decreases and increases as the thickness of the beam increases. Using alternative admissible function with penalties, these values can directly be incorporated for enforcing the Clamped-Clamped $(C C)$ boundary conditions.

The natural frequencies calculated using the proposed method for the calculation of the value of penalty functions are compared to the closed form Euler-Bernoulli beam frequencies and natural frequencies obtained using penalty values adopted from the literature. Table 4-26 to Table 4-35 tabulate the comparison and validation of results for different dimensions as indicated in Table 4-26. From Table 4-27 to Table 4-29, the length of the beam increased from $0.5 \mathrm{~m}$ to $1.5 \mathrm{~m}$ with an increment of $0.5 \mathrm{~m}$. The thickness of the beam is kept constant at $3 \mathrm{~mm}$. Table 4-27 tabulates the predicted natural frequencies using the estimated value of penalty function of $k_{0}=$ $1 \mathrm{X} 10^{7.58}$ for a beam length of $0.5 \mathrm{~m}$ and $3 \mathrm{~mm}$ thickness. Similarly, the Table 4-27 and Table 4-28 accommodate the predicted natural frequency corresponding to estimated penalty functions of $k_{0}$ $=1 \mathrm{X} 10^{6.67}$ and $k_{0}=1 \mathrm{X} 10^{6.14}$ for $1 \mathrm{~m}$ and $1.5 \mathrm{~m}$ beam length respectively. Again, In tables from 
Table 4-27 and Table 4-28 the value of penalty is considered as $k_{0}=1 \times 10^{10}$ as stated in previous literature [2], [3], [11] for the purpose of validation of the present approach.

Table 4-27: Natural Frequencies of $C C$ Beam with $L=0.5 \mathrm{~m}$ and $h=3 \mathrm{~mm}$

\begin{tabular}{|c|c|r|r|r|r|r|r|}
\hline \multicolumn{2}{|c|}{$N=12$} & \multicolumn{3}{c|}{$\boldsymbol{k}_{\mathbf{0}}=\mathbf{1 X 1 0}^{\mathbf{1 0}}$} & \multicolumn{3}{c|}{$\boldsymbol{k}_{\mathbf{0}}=\mathbf{1 X 1 0}^{\mathbf{7 . 5 8}}$} \\
\hline Mode & $\begin{array}{c}\text { EBB } \\
(\mathbf{H z})\end{array}$ & $\begin{array}{c}\text { AAF } \\
(\mathbf{H z})\end{array}$ & $\begin{array}{c}\text { \% } \\
\text { Error }\end{array}$ & $\begin{array}{c}\text { Dev. } \\
(\mathbf{H z})\end{array}$ & $\begin{array}{c}\text { AAF } \\
(\mathbf{H z})\end{array}$ & $\begin{array}{c}\text { \% } \\
\text { Error }\end{array}$ & $\begin{array}{c}\text { Dev. } \\
(\mathbf{H z})\end{array}$ \\
\hline $1^{\text {st }}$ & 10.04 & 10.04 & 0.00 & 0.00 & 10.04 & 0.00 & 0.00 \\
\hline $2^{\text {nd }}$ & 62.94 & 62.96 & 0.04 & 0.02 & 62.96 & 0.03 & 0.02 \\
\hline $3^{\text {rd }}$ & 176.23 & 176.40 & 0.10 & 0.17 & 176.37 & 0.08 & 0.13 \\
\hline $4^{\text {th }}$ & 345.35 & 346.04 & 0.20 & 0.70 & 345.90 & 0.16 & 0.56 \\
\hline $5^{\text {th }}$ & 570.88 & 572.61 & 0.30 & 1.73 & 572.24 & 0.24 & 1.36 \\
\hline $6^{\text {th }}$ & 852.80 & 857.55 & 0.56 & 4.76 & 856.76 & 0.46 & 3.96 \\
\hline
\end{tabular}

Table 4-28: Natural Frequencies of $C C$ Beam with $L=1.0 \mathrm{~m}$ and $h=3 \mathrm{~mm}$

\begin{tabular}{|c|c|r|r|r|r|r|r|}
\hline \multicolumn{2}{|c|}{$N=\mathbf{1 2}$} & \multicolumn{3}{c|}{$\boldsymbol{k}_{\mathbf{0}}=\mathbf{1 X 1 0}^{\mathbf{1 0}}$} & \multicolumn{3}{c|}{$\boldsymbol{k}_{\mathbf{0}}=\mathbf{1 X 1 0}^{\mathbf{6 . 6 7}}$} \\
\hline Mode & $\begin{array}{c}\text { EBB } \\
(\mathbf{H z})\end{array}$ & $\begin{array}{c}\text { AAF } \\
(\mathbf{H z})\end{array}$ & $\begin{array}{c}\text { \% } \\
\text { Error }\end{array}$ & $\begin{array}{c}\text { Dev. } \\
(\mathbf{H z})\end{array}$ & $\begin{array}{c}\text { AAF } \\
(\mathbf{H z})\end{array}$ & $\begin{array}{c}\text { \% } \\
\text { Error }\end{array}$ & $\begin{array}{c}\text { Dev. } \\
(\mathbf{H z})\end{array}$ \\
\hline $1^{\text {st }}$ & 2.51 & 2.51 & 0.00 & 0.00 & 2.51 & 0.00 & 0.00 \\
\hline $2^{\text {nd }}$ & 15.73 & 15.74 & 0.04 & 0.01 & 15.74 & 0.03 & 0.00 \\
\hline $3^{\text {rd }}$ & 44.06 & 44.10 & 0.10 & 0.04 & 44.09 & 0.08 & 0.03 \\
\hline $4^{\text {th }}$ & 86.34 & 86.51 & 0.20 & 0.17 & 86.48 & 0.16 & 0.14 \\
\hline $5^{\text {th }}$ & 142.72 & 143.15 & 0.30 & 0.43 & 143.06 & 0.24 & 0.34 \\
\hline $6^{\text {th }}$ & 213.20 & 214.39 & 0.56 & 1.19 & 214.19 & 0.46 & 0.99 \\
\hline
\end{tabular}


Table 4-29: Natural Frequencies of $C C$ Beam with $L=1.5 \mathrm{~m}$ and $h=3 \mathrm{~mm}$

\begin{tabular}{|c|c|r|r|r|r|r|r|}
\hline \multicolumn{2}{|c|}{$N=\mathbf{1 2}$} & \multicolumn{3}{c|}{$\boldsymbol{k}_{\mathbf{0}}=\mathbf{1 X 1 0}$} & \multicolumn{3}{c|}{$\boldsymbol{k}_{\mathbf{0}}=\mathbf{1 X 1 0}^{\mathbf{6}} \mathbf{1 4}$} \\
\hline Mode & $\begin{array}{c}\text { EBB } \\
(\mathbf{H z})\end{array}$ & $\begin{array}{c}\text { AAF } \\
(\mathbf{H z})\end{array}$ & $\begin{array}{c}\text { \% } \\
\text { Error }\end{array}$ & $\begin{array}{c}\text { Dev. } \\
(\mathbf{H z})\end{array}$ & $\begin{array}{c}\text { AAF } \\
(\mathbf{H z})\end{array}$ & $\begin{array}{c}\text { \% } \\
\text { Error }\end{array}$ & $\begin{array}{c}\text { Dev. } \\
(\mathbf{H z})\end{array}$ \\
\hline $1^{\text {st }}$ & 1.12 & 1.12 & 0.00 & 0.00 & 1.12 & 0.00 & 0.00 \\
\hline $2^{\text {nd }}$ & 6.99 & 7.00 & 0.04 & 0.00 & 7.00 & 0.03 & 0.00 \\
\hline $3^{\text {rd }}$ & 19.58 & 19.60 & 0.10 & 0.02 & 19.60 & 0.08 & 0.01 \\
\hline $4^{\text {th }}$ & 38.37 & 38.45 & 0.20 & 0.08 & 38.43 & 0.16 & 0.06 \\
\hline $5^{\text {th }}$ & 63.43 & 63.62 & 0.30 & 0.19 & 63.58 & 0.24 & 0.15 \\
\hline $6^{\text {th }}$ & 94.76 & 95.28 & 0.56 & 0.53 & 95.20 & 0.46 & 0.44 \\
\hline
\end{tabular}

From Table 4-27 to Table 4-29, the percentage error in predicting the natural frequency with estimated penalty value decreases compared to the natural frequency obtained with the value of penalty selected from previous literature.

Table 4-30 to Table 4-32, tabulate the natural frequencies of beam length of $0.5 \mathrm{~m}$ to $1.5 \mathrm{~m}$ with a constant thickness of $4 \mathrm{~mm}$.

Table 4-30: Natural Frequencies of $C C$ Beam with $L=0.5 \mathrm{~m}$ and $h=4 \mathrm{~mm}$

\begin{tabular}{|c|c|r|r|r|r|r|r|}
\hline \multicolumn{2}{|c|}{$N=\mathbf{1 2}$} & \multicolumn{3}{c|}{$\boldsymbol{k}_{\mathbf{0}}=\mathbf{1 X 1 0}^{10}$} & \multicolumn{3}{c|}{$\boldsymbol{k}_{\mathbf{0}}=\mathbf{1 X 1 0}$} \\
\hline Mode & $\begin{array}{c}\text { EBB } \\
(\mathbf{H z})\end{array}$ & $\begin{array}{c}\text { AAF } \\
(\mathbf{H z})\end{array}$ & $\begin{array}{c}\text { \% } \\
\text { Error }\end{array}$ & $\begin{array}{c}\text { Dev. } \\
(\mathbf{H z})\end{array}$ & $\begin{array}{c}\text { AAF } \\
(\mathbf{H z})\end{array}$ & $\begin{array}{c}\text { \% } \\
\text { Error }\end{array}$ & $\begin{array}{c}\text { Dev. } \\
(\mathbf{H z})\end{array}$ \\
\hline $1^{\text {st }}$ & 13.39 & 13.39 & 0.00 & 0.00 & 13.39 & 0.00 & 0.00 \\
\hline $2^{\text {nd }}$ & 83.92 & 83.95 & 0.04 & 0.03 & 83.94 & 0.03 & 0.02 \\
\hline $3^{\text {rd }}$ & 234.98 & 235.20 & 0.10 & 0.23 & 235.15 & 0.08 & 0.18 \\
\hline $4^{\text {th }}$ & 460.46 & 461.39 & 0.20 & 0.93 & 461.20 & 0.16 & 0.74 \\
\hline $5^{\text {th }}$ & 761.17 & 763.48 & 0.30 & 2.31 & 762.99 & 0.24 & 1.82 \\
\hline $6^{\text {th }}$ & 1137.06 & 1143.40 & 0.56 & 6.34 & 1142.34 & 0.46 & 5.28 \\
\hline
\end{tabular}


Table 4-31: Natural Frequencies of CC Beam with $\mathrm{L}=1.0 \mathrm{~m}$ and $\mathrm{h}=4 \mathrm{~mm}$

\begin{tabular}{|c|c|r|r|r|r|r|r|}
\hline \multicolumn{2}{|c|}{$N=\mathbf{1 2}$} & \multicolumn{3}{c|}{$\boldsymbol{k}_{\mathbf{0}}=\mathbf{1 X 1 0}^{10}$} & \multicolumn{3}{c|}{$\boldsymbol{k}_{\mathbf{0}}=\mathbf{1 X 1 0}^{\mathbf{7 0 5}}$} \\
\hline Mode & $\begin{array}{c}\text { EBB } \\
(\mathbf{H z})\end{array}$ & $\begin{array}{c}\text { AAF } \\
(\mathbf{H z})\end{array}$ & $\begin{array}{c}\text { \% } \\
\text { Error }\end{array}$ & $\begin{array}{c}\text { Dev. } \\
(\mathbf{H z})\end{array}$ & $\begin{array}{c}\text { AAF } \\
(\mathbf{H z})\end{array}$ & $\begin{array}{c}\text { \% } \\
\text { Error }\end{array}$ & $\begin{array}{c}\text { Dev. } \\
(\mathbf{H z})\end{array}$ \\
\hline $1^{\text {st }}$ & 3.35 & 3.35 & 0.00 & 0.00 & 3.35 & 0.00 & 0.00 \\
\hline $2^{\text {nd }}$ & 20.98 & 20.99 & 0.04 & 0.01 & 20.99 & 0.03 & 0.01 \\
\hline $3^{\text {rd }}$ & 58.74 & 58.80 & 0.10 & 0.06 & 58.79 & 0.08 & 0.04 \\
\hline $4^{\text {th }}$ & 115.12 & 115.35 & 0.20 & 0.23 & 115.30 & 0.16 & 0.19 \\
\hline $5^{\text {th }}$ & 190.29 & 190.87 & 0.30 & 0.58 & 190.75 & 0.24 & 0.45 \\
\hline $6^{\text {th }}$ & 284.27 & 285.85 & 0.56 & 1.59 & 285.59 & 0.46 & 1.32 \\
\hline
\end{tabular}

Table 4-32: Natural Frequencies of $C C$ Beam with $L=1.5 \mathrm{~m}$ and $h=4 \mathrm{~mm}$

\begin{tabular}{|c|c|r|r|r|r|r|r|}
\hline \multicolumn{2}{|c|}{$N=\mathbf{1 2}$} & \multicolumn{3}{c|}{$\boldsymbol{k}_{\mathbf{0}}=\mathbf{1 X 1 0}^{\mathbf{1 0}}$} & \multicolumn{3}{c|}{$\boldsymbol{k}_{\mathbf{0}}=\mathbf{1 X 1 0}^{6.52}$} \\
\hline Mode & $\begin{array}{c}\text { EBB } \\
(\mathbf{H z})\end{array}$ & $\begin{array}{c}\text { AAF } \\
(\mathbf{H z})\end{array}$ & $\begin{array}{c}\text { \% } \\
\text { Error }\end{array}$ & $\begin{array}{c}\text { Dev. } \\
(\mathbf{H z})\end{array}$ & $\begin{array}{c}\text { AAF } \\
(\mathbf{H z})\end{array}$ & $\begin{array}{c}\text { \% } \\
\text { Error }\end{array}$ & $\begin{array}{c}\text { Dev. } \\
(\mathbf{H z})\end{array}$ \\
\hline $1^{\text {st }}$ & 1.49 & 1.49 & 0.00 & 0.00 & 1.49 & 0.00 & 0.00 \\
\hline $2^{\text {nd }}$ & 9.32 & 9.33 & 0.04 & 0.00 & 9.33 & 0.03 & 0.00 \\
\hline $3^{\text {rd }}$ & 26.11 & 26.13 & 0.10 & 0.03 & 26.13 & 0.08 & 0.02 \\
\hline $4^{\text {th }}$ & 51.16 & 51.27 & 0.20 & 0.10 & 51.24 & 0.16 & 0.08 \\
\hline $5^{\text {th }}$ & 84.57 & 84.83 & 0.30 & 0.26 & 84.78 & 0.24 & 0.20 \\
\hline $6^{\text {th }}$ & 126.34 & 127.05 & 0.56 & 0.71 & 126.93 & 0.46 & 0.59 \\
\hline
\end{tabular}

From Table 4-33 to Table 4-35 the thickness of the beam is kept constant at $5 \mathrm{~mm}$ and the corresponding values of estimated penalty functions for different length are tabulated. 
Table 4-33: Natural Frequencies of $C C$ Beam with $L=0.5 \mathrm{~m}$ and $h=5 \mathrm{~mm}$

\begin{tabular}{|c|c|c|c|c|c|c|c|}
\hline \multicolumn{2}{|c|}{$N=\mathbf{1 2}$} & \multicolumn{3}{c|}{$\boldsymbol{k}_{\mathbf{0}}=\mathbf{1 X 1 0}^{10}$} & \multicolumn{3}{c|}{$\boldsymbol{k}_{\mathbf{0}}=\mathbf{1 X 1 0}^{8.24}$} \\
\hline Mode & $\begin{array}{c}\text { EBB } \\
(\mathbf{H z})\end{array}$ & $\begin{array}{c}\text { AAF } \\
(\mathbf{H z})\end{array}$ & $\begin{array}{c}\text { \% } \\
\text { Error }\end{array}$ & $\begin{array}{c}\text { Dev. } \\
(\mathbf{H z})\end{array}$ & $\begin{array}{c}\text { AAF } \\
(\mathbf{H z})\end{array}$ & $\begin{array}{c}\text { \% } \\
\text { Error }\end{array}$ & $\begin{array}{c}\text { Dev. } \\
(\mathbf{H z})\end{array}$ \\
\hline $1^{\text {st }}$ & 16.74 & 16.74 & 0.00 & 0.00 & 16.74 & 0.00 & 0.00 \\
\hline $2^{\text {nd }}$ & 104.90 & 104.94 & 0.04 & 0.04 & 104.93 & 0.03 & 0.03 \\
\hline $3^{\text {rd }}$ & 293.72 & 294.00 & 0.10 & 0.28 & 293.94 & 0.08 & 0.22 \\
\hline $4^{\text {th }}$ & 575.58 & 576.73 & 0.20 & 1.16 & 576.51 & 0.16 & 0.93 \\
\hline $5^{\text {th }}$ & 951.47 & 954.35 & 0.30 & 2.88 & 953.74 & 0.24 & 2.27 \\
\hline $6^{\text {th }}$ & 1421.33 & 1429.24 & 0.56 & 7.91 & 1427.93 & 0.46 & 6.60 \\
\hline
\end{tabular}

Table 4-34: Natural Frequencies of $C C$ Beam with $L=1.0 \mathrm{~m}$ and $h=5 \mathrm{~mm}$

\begin{tabular}{|c|c|r|r|r|r|r|r|}
\hline \multicolumn{2}{|c|}{$N=\mathbf{1 2}$} & \multicolumn{3}{c|}{$\boldsymbol{k}_{\mathbf{0}}=\mathbf{1 X 1 0}^{\mathbf{1 0}}$} & \multicolumn{3}{c|}{$\boldsymbol{k}_{\mathbf{0}}=\mathbf{1 X 1 0}^{\mathbf{7 . 3 4}}$} \\
\hline Mode & $\begin{array}{c}\text { EBB } \\
(\mathbf{H z})\end{array}$ & $\begin{array}{c}\text { AAF } \\
(\mathbf{H z})\end{array}$ & $\begin{array}{c}\text { \% } \\
\text { Error }\end{array}$ & $\begin{array}{c}\text { Dev. } \\
(\mathbf{H z})\end{array}$ & $\begin{array}{c}\text { AAF } \\
(\mathbf{H z})\end{array}$ & $\begin{array}{c}\text { \% } \\
\text { Error }\end{array}$ & $\begin{array}{c}\text { Dev. } \\
(\mathbf{H z})\end{array}$ \\
\hline $1^{\text {st }}$ & 4.18 & 4.18 & 0.00 & 0.00 & 4.18 & 0.00 & 0.00 \\
\hline $2^{\text {nd }}$ & 26.22 & 26.23 & 0.04 & 0.01 & 26.23 & 0.03 & 0.01 \\
\hline $3^{\text {rd }}$ & 73.43 & 73.50 & 0.10 & 0.07 & 73.49 & 0.08 & 0.06 \\
\hline $4^{\text {th }}$ & 143.89 & 144.18 & 0.20 & 0.29 & 144.13 & 0.16 & 0.23 \\
\hline $5^{\text {th }}$ & 237.87 & 238.59 & 0.30 & 0.72 & 238.43 & 0.24 & 0.57 \\
\hline $6^{\text {th }}$ & 355.33 & 357.31 & 0.56 & 1.98 & 356.98 & 0.46 & 1.65 \\
\hline
\end{tabular}


Table 4-35: Natural Frequencies of $C C$ Beam with $L=1.5 \mathrm{~m}$ and $h=5 \mathrm{~mm}$

\begin{tabular}{|c|c|r|r|r|r|r|r|}
\hline \multicolumn{2}{|c|}{$N=\mathbf{1 2}$} & \multicolumn{3}{c|}{$\boldsymbol{k}_{\mathbf{0}}=\mathbf{1 X 1 0}^{\mathbf{1 0}}$} & \multicolumn{3}{c|}{$\boldsymbol{k}_{\mathbf{0}}=\mathbf{1 X 1 0}^{\mathbf{6 . 8 1}}$} \\
\hline Mode & $\begin{array}{c}\text { EBB } \\
(\mathbf{H z})\end{array}$ & $\begin{array}{c}\text { AAF } \\
(\mathbf{H z})\end{array}$ & $\begin{array}{c}\text { \% } \\
\text { Error }\end{array}$ & $\begin{array}{c}\text { Dev. } \\
(\mathbf{H z})\end{array}$ & $\begin{array}{c}\text { AAF } \\
(\mathbf{H z})\end{array}$ & $\begin{array}{c}\text { \% } \\
\text { Error }\end{array}$ & $\begin{array}{c}\text { Dev. } \\
(\mathbf{H z})\end{array}$ \\
\hline $1^{\text {st }}$ & 1.86 & 1.86 & 0.00 & 0.00 & 1.86 & 0.00 & 0.00 \\
\hline $2^{\text {nd }}$ & 11.66 & 11.66 & 0.04 & 0.00 & 11.66 & 0.03 & 0.00 \\
\hline $3^{\text {rd }}$ & 32.64 & 32.67 & 0.10 & 0.03 & 32.66 & 0.08 & 0.02 \\
\hline $4^{\text {th }}$ & 63.95 & 64.08 & 0.20 & 0.13 & 64.06 & 0.16 & 0.10 \\
\hline $5^{\text {th }}$ & 105.72 & 106.04 & 0.30 & 0.32 & 105.97 & 0.24 & 0.25 \\
\hline $6^{\text {th }}$ & 157.93 & 158.81 & 0.56 & 0.88 & 158.66 & 0.46 & 0.73 \\
\hline
\end{tabular}

It is noticed in all three boundary conditions, the percentage of error in predicting the natural frequency obtained using the estimated penalty estimated with the proposed methodology is lower as compared to when using the penalty values adopted directly from literature. The estimated value of penalties provides better convergence in predicting the natural frequency to the closed-form solution. The proposed method in obtaining the penalty functions is a systematic approach and yields good results. Using the proposed methodology, the estimation of the penalty function for any given beam parameter is easily done. 


\subsection{Conclusion}

In this chapter, an attempt has been made to calculate the penalty values for different boundary conditions. The values of the penalty function not only depend on the type of boundary conditions, but also on the system parameters. A procedure to calculate the penalty values for three different boundary conditions was proposed, viz., $C F, S S$, and $C C$.

It was noticed that in the three cases, the specific value required for penalty function is directly proportional to the Young's modulus $(E)$ of the beam material, second moment of area of the cross section $(I)$ and inversely proportional the length $(L)$ of the beam used. As a part of this conclusion the following relations for the aforementioned three boundary conditions are proposed as

$$
\begin{array}{lc}
\text { Boundary Conditions } & \text { Penalty function } \\
\text { Clamped-Free }(C F) & k_{0}=k_{r 0}=\frac{E I}{L^{3}} \times 10^{5.49} \\
\text { Simply-Supported }(S S) & k_{0}=k_{r 0}=\frac{E I}{L^{3}} \times 10^{5.03} \\
\text { Clamped-Clamped }(C C) & k_{0}=k_{r 0}=\frac{E I}{L^{3}} \times 10^{5.78}
\end{array}
$$

The developed generalized expressions were successfully validated with numerical examples for different boundary conditions and geometrical properties. The natural frequencies obtained using the $A A F$ method with penalties estimated with this approach are in excellent agreement with the benchmark closed-form Euler-Bernoulli values, as compared to those obtained using penalty values selected from previous literature. The $A A F$ with penalty can be used with different boundary conditions. The same approach can be used to estimate the penalty values as well. 


\section{Alternative Admissible Function Approach for a Beam with a}

\section{Shallow Crack}

\subsection{Introduction}

Vibration analysis of structures with cracks is an interesting topic in many disciplines of study. A beam is one of these fundamental structures. It is important to investigate the influence of a crack on structures to avoid catastrophic failures due to a crack. The presence of a crack causes a change in the structure's local stiffness, and hence a change in the structure's dynamic behaviour, such as natural frequencies and mode shapes [13],[14],[15],[16],[17].

In this chapter, an analytical method to determine the eigenvalues and eigenfunctions of a beam with a shallow is proposed. The crack is modelled as a penalty function to account for the local stiffness reduction. From Chapter 3 and 4, the mass, stiffness, and penalty function matrices for various boundary conditions were developed and are used for the analysis of a beam with a crack. Due to the presence of the crack, the stiffness matrix of the unconstrained beam is altered, and the effective stiffness of the beam with a crack is calculated. The eigenvalues and eigenfunctions for the beam with a shallow crack for various locations and crack depth ratios are determined by constructing the equation of motion.

The study is conducted for three typical boundary conditions: Simply-Supported (SS), Clamped-Clamped $(C C)$, and Clamped-Free $(C F)$. Finally, the last section is devoted to validating the results obtained using the developed model with published literature. 


\subsection{Formulation of Crack as a Penalty}

The alternative admissible function of the uncracked beam is used to model the assumed modes of the beam with a shallow crack for a Rayleigh-Ritz approximation. The presence of a crack leads to loss of potential energy [27] and is included by modifying the potential energy of the cracked beam. The energy loss is proportional to the amount of added flexibility in the beam. To quantify the added flexibility, the crack is modeled as a penalty function. The virtual linear stiffness at the location of the crack is assumed to be negligible and the virtual rotational stiffness at the location of the crack is considered as a massless rotational spring $\left(k_{c}\right)$.

Figure 5-1 shows a beam with shallow crack at a distance $x_{c}$ from one end of the beam. The dimensions of the beam are represented by length $L$, breadth $b$ and thickness $h$.

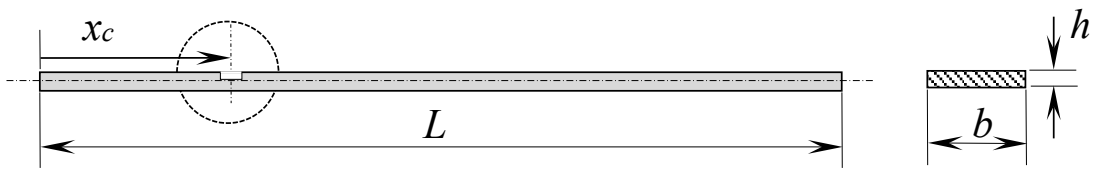

Figure 5-1: A Euler-Bernoulli Beam with a Shallow Crack at $x_{c}$

The magnified view of the location of the shallow crack in the beam is shown in Figure $5-2$, indicating the depth of the crack as $d_{c}$. Only bending vibrations is considered in the present work.

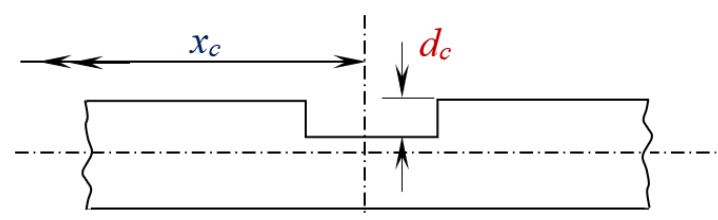

Figure 5-2: Magnified View of Crack on the Beam

Dimarogonas [41] defined the torsional spring constant in the vicinity of the cracked section of a beam when a lateral crack of uniform depth exists. The crack is represented as a massless rotational spring in literature [18]-[25] due to its simplicity and adaptability. Afshari and Inman [27] have 
represented a crack as a massless rotational spring in the modal analysis of a simply-supported and clamped-clamped cracked beam as shown in Figure 5-3.

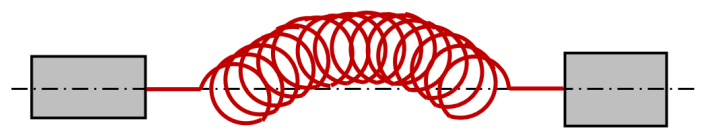

Figure 5-3: Crack Represented as a Massless Rotational Spring

The potential energy due to the presence of the crack can be quantified as stated in Afshari and Inman [27]

$$
V_{c}=\frac{1}{2} E I C_{E}\left(\frac{\partial^{2} W}{\partial x^{2}}\left(x_{c}\right)\right)^{2}
$$

where effective compliance of crack $C_{E}$, is given as $C_{E}=E I C_{c} . E$ and $I$ are the known beam properties (Young's modulus and area moment of inertia), and $\left(C_{c}\right)$ is the compliance of the crack.

The potential energy is proportional to the added compliance due to the presence of the crack and the added rotation. The compliance of the $\operatorname{crack}\left(C_{c}\right)$ of the virtual rotational stiffness at the location of the crack, as given in Afshari and Inman approach [27] is

$$
C_{c}=\frac{1}{K_{c}}=\frac{6 h \pi}{E I} \alpha\left(\frac{d_{c}}{h}\right)
$$

where, $E I$ is the flexural rigidity of the beam, $h$ is the thickness of the beam and $d_{c}$ represents the depth of the crack. In the present study, the values of the function $\alpha$ for different crack depth ratios in the range of $0 \leq \frac{d_{c}}{h} \leq 0.6$ is considered from Afshari and Inman [27] and is expressed as

$$
\begin{aligned}
& \alpha\left(\frac{d_{c}}{h}\right)=0.629\left(\frac{d_{c}}{h}\right)^{2}-1.047\left(\frac{d_{c}}{h}\right)^{3}+4.602\left(\frac{d_{c}}{h}\right)^{4}-9.975\left(\frac{d_{c}}{h}\right)^{5}+ \\
& 20.295\left(\frac{d_{c}}{h}\right)^{6}-32.993\left(\frac{d_{c}}{h}\right)^{7}+47.041\left(\frac{d_{c}}{h}\right)^{8}-40.693\left(\frac{d_{c}}{h}\right)^{9}+19.6\left(\frac{d_{c}}{h}\right)^{10}
\end{aligned}
$$


The (modified) potential energy of the cracked beam is formulated by subtracting the potential energy due to the presence of the crack in Eq. (5.1) from the potential energy of the unconstrained beam. The modified potential energy of the beam in the presence of crack is then given by

$$
V_{\text {mod }}=\frac{E I}{2} \int_{x=0}^{x=L}\left(\frac{\partial^{2} W}{\partial x^{2}}\right)^{2} d x-\frac{1}{2} E I C_{E}\left(\frac{\partial^{2} W}{\partial x^{2}}\left(x_{c}\right)\right)^{2}
$$

Applying the Rayleigh-Ritz minimization to the conservative system, using the maximum modified potential energy, leads to the effective stiffness matrices for the traverse vibrations of the cracked beam

$$
k_{E, i j}=E I \int_{0}^{L} \phi_{i}{ }^{\prime \prime}(x) \phi_{j}{ }^{\prime \prime}(x) d x-E I C_{E}\left[\phi_{i}{ }^{\prime \prime}\left(x_{c}\right)\right]\left[\phi_{j}{ }^{\prime \prime}\left(x_{c}\right)\right]
$$

The first part of the Eq. (5.5) accounts for the unconstrained beam stiffness and the later part accounts for the reduction in stiffness due the presence of the crack. The stiffness matrix of the cracked beam portion is expressed as,

$$
k_{c, i j}=\operatorname{EIC}_{E}\left[\phi^{i^{\prime \prime}}\left(x_{c}\right)\right]\left[\phi^{j^{\prime \prime}}\left(x_{c}\right)\right]
$$

Equation (5.6) can be expressed in matrix form for $N=5$ as,

$$
\begin{aligned}
& K_{C} \\
& =E I C_{E}\left[\begin{array}{ccccc}
0 & 0 & 0 & 0 & 0 \\
0 & 0 & 0 & 0 & 0 \\
0 & 0 & 4 & -2 \pi^{2} \cos \left(\pi \frac{x_{c}}{L}\right) & -8 \pi^{2} \cos \left(2 \pi \frac{x_{c}}{L}\right) \\
0 & 0 & -2 \pi^{2} \cos \left(\pi \frac{x_{c}}{L}\right) & \pi^{4} \cos \left(\pi \frac{x_{c}}{L}\right)^{2} & 4 \pi^{4} \cos \left(\pi \frac{x_{c}}{L}\right) \cos \left(2 \pi \frac{x_{c}}{L}\right) \\
0 & 0 & -8 \pi^{2} \cos \left(2 \pi \frac{x_{c}}{L}\right) & 4 \pi^{4} \cos \left(\pi \frac{x_{c}}{L}\right) \cos \left(2 \pi \frac{x_{c}}{L}\right) & 16 \pi^{4} \cos \left(2 \pi \frac{x_{c}}{L}\right)^{2}
\end{array}\right]
\end{aligned}
$$

Using the (5.7), the equation of motion for a beam with shallow crack is developed in the following sections. 


\subsection{Equation of Motion of a Beam with a Shallow Crack}

The frequency equation for a beam with shallow crack takes the generalized matrix form as

$$
\left[\left(\left(K-K_{c}\right)+K_{P}\right)-\omega^{2} M\right] C=0
$$

Eq. (5.8) can be simplified as

$$
\left[\left(K_{E}+K_{P}\right)-\omega^{2} M\right] C=0
$$

where $K$ and $M$ are the generalized unconstrained beam stiffness and mass matrices of size $N$ x $N$, respectively, $K_{P}$ is the penalty matrix depending on the type of boundary to be enforced, $K_{c}$ is the stiffness matrix due to the presence of the crack, the vector $C$ is the coefficients from Eq. (3.2) and $K_{E}=\left(K-K_{c}\right)$ is the effective stiffness matrix of the cracked beam (beam only, without boundary conditions considered) and is given by

$$
K_{E}=\frac{E I}{L^{3}}\left[\begin{array}{ccccc}
0 & 0 & 0 & 0 & 0 \\
0 & 0 & 0 & 0 & 0 \\
0 & 0 & 4(1-\gamma) & 2 \pi^{2} \cos \left(\pi \frac{x_{c}}{L}\right) \gamma & 8 \pi^{2} \cos \left(2 \pi \frac{x_{c}}{L}\right) \gamma \\
0 & 0 & 2 \pi^{2} \cos \left(\pi \frac{x_{c}}{L}\right) \gamma & \frac{\pi^{4}}{2}\left(1-2 \cos \left(\pi \frac{x_{c}}{L}\right)^{2} \gamma\right) & -4 \pi^{4} \cos \left(\pi \frac{x_{c}}{L}\right) \cos \left(2 \pi \frac{x_{c}}{L}\right) \gamma \\
0 & 0 & 8 \pi^{2} \cos \left(2 \pi \frac{x_{c}}{L}\right) \gamma & -4 \pi^{4} \cos \left(\pi \frac{x_{c}}{L}\right) \cos \left(2 \pi \frac{x_{c}}{L}\right) \gamma & \frac{(2 \pi)^{4}}{2}\left(1-2 \cos \left(2 \pi \frac{x_{c}}{L}\right)^{2} \gamma\right)
\end{array}\right]
$$

and where $\gamma=\frac{6 \pi h}{L} \alpha\left(\frac{d_{c}}{h}\right)$

It can be noticed that the effective stiffness matrix in Eq. (5.10) involves the location of the crack,

$x_{c}$, and as well as the crack depth ratio, $\frac{d_{c}}{h}$. The advantage of modelling crack as a penalty is that it can easily be enforced in a beam with different boundary conditions directly for any location of crack and crack depth ratio.

For a non-trivial solution of Eq. (5.9), the determinant of the coefficients of $C$ must be equal to zero, hence

$$
\left|K_{E}+K_{P}-\omega^{2} M\right|=0
$$


Eq. (5.11) can be used to estimate the natural frequencies of a beam with a crack at different location and depth ratio.

\subsection{Validation of Proposed Approach}

Using Eq. (5.11), the first three natural frequencies and modeshapes of a beam with a shallow crack are extracted for different location and crack depth ratio. The results obtained are validated with the available literature results.

\subsubsection{Crack Parameters}

The validation for the present approach is carried out using the geometrical parameters and material properties of Afshari and Inman [27]. The data available in Afshari and Inman [27] are limited to Simply-Supported (SS) and Clamped-Clamped (CC) boundary conditions. The geometrical parameters and material properties are tabulated in Table 5-1.

Table 5-1: Geometrical Parameters and Material Properties

\begin{tabular}{|l|r|}
\hline Young's Modulus $(\mathrm{E})$ & $71.7 \mathrm{GPa}$ \\
\hline Mass Density $(\rho)$ & $2770 \mathrm{~kg} / \mathrm{m}^{3}$ \\
\hline Length, $L$ & $0.3048 \mathrm{~m}$ \\
\hline Thickness, $h$ & $0.0032 \mathrm{~m}$ \\
\hline Width, $b$ & $0.0254 \mathrm{~m}$ \\
\hline
\end{tabular}

The normalized crack position considered in the literature is varied from 0.2 to 0.8 times the total length of the beam. The crack depth ratio considered are from 0.2 to 0.4 . The following section provides validation of results for simply-supported and clamped-clamped cracked beam.

\subsubsection{Validation of a Shallow Crack in a Simply-Supported (SS) Beam}

The geometrical parameters and material properties tabulated in Table 5-1 is used to model a $S S$ beam. Using the Eq. (4.18) for $N=12$, the boundary penalty function for the $S S$ boundary conditions is calculated as, 


$$
\begin{aligned}
& k_{0}=k_{r 0}=\frac{E I}{L^{3}} \times 10^{5.03}=\frac{71.7 \times 10^{9} \times\left(0.0254 \times 0.0032^{3}\right)}{12 \times 0.3048^{3}} \times 10^{5.03} \\
& k_{0}=k_{r 0}=1.8818 \times 10^{7}=1 \times 10^{7.27}
\end{aligned}
$$

Upon substituting the value of $k_{0}$ in Eq. (4.13), $K_{P, S S}$ can be evaluated. Using Eq. (5.10), effective stiffness matrix $K_{E}$ of the beam with a shallow crack for the chosen system parameters is evaluated. On substituting the expression for $K_{P, S S}, K_{E}$ and $M$ in Eq. (5.11), the natural frequencies of the $S S$ beam with shallow crack can be evaluated. Table 5-2 shows the evaluated first three natural frequencies of a $S S$ beam with shallow crack for a normalized crack positions of $0.2,0.4,0.6$, and 0.8 from the end, and with a crack depth ratio of 0.2 . The second and the third column tabulates the natural frequency using the Afshari and Inman [27] approach and the proposed $A A F$ method respectively. The fourth column tabulates the percentage error in predicting the natural frequency using the present approach. 
Table 5-2: Natural Frequencies (Hz) of SS Beam with Crack Depth Ratio, $\left(d_{c} / h\right)=0.2$.

\begin{tabular}{|c|c|c|c|}
\hline Mode & $\begin{array}{c}\text { Afshari\& Inman [27] } \\
(\mathrm{Hz})\end{array}$ & $\begin{array}{l}\mathrm{AAF} \\
(\mathbf{H z})\end{array}$ & $\begin{array}{c}\% \\
\text { Error }\end{array}$ \\
\hline \multicolumn{4}{|c|}{ Normalized Location of Crack, $\left(x_{c}\right)=0.2$} \\
\hline 1 & 78.78 & 79.34 & 0.71 \\
\hline 2 & 314.40 & 316.49 & 0.67 \\
\hline 3 & 707.80 & 712.24 & 0.63 \\
\hline \multicolumn{4}{|c|}{ Normalized Location of Crack, $\left(x_{c}\right)=0.4$} \\
\hline 1 & 78.58 & 79.14 & 0.71 \\
\hline 2 & 315.20 & 317.30 & 0.67 \\
\hline 3 & 709.00 & 714.30 & 0.75 \\
\hline \multicolumn{4}{|c|}{ Normalized Location of Crack, $\left(x_{c}\right)=0.6$} \\
\hline 1 & 78.58 & 79.14 & 0.71 \\
\hline 2 & 315.2 & 317.30 & 0.67 \\
\hline 3 & 709.0 & 714.30 & 0.75 \\
\hline \multicolumn{4}{|c|}{ Normalized Location of Crack, $\left(x_{c}\right)=0.8$} \\
\hline 1 & 78.78 & 79.34 & 0.71 \\
\hline 2 & 314.4 & 316.49 & 0.67 \\
\hline 3 & 707.8 & 712.24 & 0.63 \\
\hline
\end{tabular}

It is noticed that the results obtained are in good agreement with the results considered for comparison from Afshari and Inman approach [27] and the errors are less than 1\%. As expected, due to symmetry, the results for pairs of cracks present at normalized positions of $0.2 \& 0.8$, and $0.4 \& 0.6$, are the same. It is noted that for the first mode, there is a dip in the natural frequency as the location of crack moves towards the centre of the beam.

Similarly, the natural frequencies for crack depth ratio of 0.4 at four different normalized crack positions are obtained and tabulated in Table 5-3. 
Table 5-3: Natural Frequencies (Hz) of SS Beam with Crack Depth Ratio, $\left(d_{c} / h\right)=0.4$

\begin{tabular}{|c|c|c|c|}
\hline Mode & $\begin{array}{c}\text { Afshari and Inman } \\
{[27]} \\
(\mathrm{Hz})\end{array}$ & $\begin{array}{l}\mathbf{A A F} \\
(\mathbf{H z})\end{array}$ & $\begin{array}{c}\text { \%o } \\
\text { Error }\end{array}$ \\
\hline \multicolumn{4}{|c|}{ Normalized Location of Crack, $\left(x_{c}\right)=0.2$} \\
\hline 1 & 78.37 & 78.78 & 0.53 \\
\hline 2 & 309.90 & 311.02 & 0.36 \\
\hline 3 & 697.00 & 700.04 & 0.44 \\
\hline \multicolumn{4}{|c|}{ Normalized Location of Crack, $\left(x_{c}\right)=0.4$} \\
\hline 1 & 77.50 & 77.75 & 0.32 \\
\hline 2 & 313.5 & 315.26 & 0.56 \\
\hline 3 & 705.0 & 710.11 & 0.72 \\
\hline \multicolumn{4}{|c|}{ Normalized Location of Crack, $\left(x_{c}\right)=0.6$} \\
\hline 1 & 77.50 & 77.75 & 0.32 \\
\hline 2 & 313.5 & 315.26 & 0.56 \\
\hline 3 & 705.0 & 710.11 & 0.72 \\
\hline \multicolumn{4}{|c|}{ Normalized Location of Crack, $\left(x_{c}\right)=0.8$} \\
\hline 1 & 78.37 & 78.78 & 0.53 \\
\hline 2 & 309.90 & 311.02 & 0.36 \\
\hline 3 & 697.00 & 700.04 & 0.44 \\
\hline
\end{tabular}

It is noticed that estimated natural frequencies using $A A F$ method are in good agreement with the reported literature. It can be observed that the percentage error in predicting the natural frequency is larger for the first mode as the normalized location of the crack moves closer to the boundaries compared to the second and third mode. This type of trend could be due to the selected boundary penalty value. In the present study, the boundary penalty value for both uncracked and cracked beam was chosen to be the same. As the predicted natural frequency using $A A F$ approach 
is higher for the first mode compared Afshari and Inman [27], this indicated the selected boundary penalty value is higher and can be tuned by considering the crack. It can be concluded that the present method can predict the natural frequencies for different normalized positions and crackdepth ratio. The percentage of error is less than $1 \%$ and has a similar type of trend about the centre of the beam, as indicated for a crack depth ratio of 0.2 . The next section reports and discusses the validation of results obtained for $C C$ boundary conditions.

\subsubsection{Validation of a Shallow Crack in a Clamped-Clamped (CC) Beam}

Having obtained a good agreement in results for SS boundary condition, the work is extended to validate the results for $C C$ boundary conditions using the $A A F$ method. The geometrical parameters and material properties tabulated in Table 5-1 is used to model a $C C$ beam. Using the Eq. (4.24) for $N=12$, the penalty function is calculated as,

$$
\begin{aligned}
& k_{0}=k_{r 0}=\frac{E I}{L^{3}} \times 10^{5.78}=\frac{71.7 \times 10^{9} \times\left(0.0254 \times 0.0032^{3}\right)}{12 \times 0.3048^{3}} \times 10^{5.78} \\
& k_{0}=k_{r 0}=1.0582 \times 10^{8}=1 \times 10^{8.02}
\end{aligned}
$$

Upon substituting the value of $k_{0}$ in Eq. (4.19), $K_{P, C C}$ can be evaluated.

Using Eq. (5.10), effective stiffness matrix $K_{E}$ of the beam with a shallow crack for the present system parameters are evaluated. On substituting the expression for $K_{P, C C}, K_{E}$ and $M$ in Eq. (5.11), the natural frequencies of the $S S$ beam with a shallow crack can be evaluated. Table 5-4 tabulates the first three natural frequencies of $C C$ beam with shallow crack for a normalized crack positions of $0.2,0.4,0.6$, and 0.8 from one end, with a crack depth ratio of 0.2 . 
Table 5-4: Natural Frequencies (Hz) of CC Beam with Crack Depth Ratio, $\left(d_{c} / h\right)=0.2$

\begin{tabular}{|c|c|c|c|}
\hline Mode & $\begin{array}{c}\text { Afshari and Inman [27] } \\
(\mathrm{Hz})\end{array}$ & $\begin{array}{l}\mathrm{AAF} \\
(\mathbf{H z})\end{array}$ & $\begin{array}{c}\% \\
\text { Error }\end{array}$ \\
\hline \multicolumn{4}{|c|}{ Normalized Location of Crack, $\left(x_{c}\right)=0.2$} \\
\hline 1 & 178.80 & 180.24 & 0.81 \\
\hline 2 & 492.50 & 496.44 & 0.80 \\
\hline 3 & 964.00 & 972.99 & 0.93 \\
\hline \multicolumn{4}{|c|}{ Normalized Location of Crack, $\left(x_{c}\right)=0.4$} \\
\hline 1 & 178.50 & 179.79 & 0.72 \\
\hline 2 & 492.00 & 496.12 & 0.84 \\
\hline 3 & 963.00 & 976.20 & 1.37 \\
\hline \multicolumn{4}{|c|}{ Normalized Location of Crack, $\left(x_{c}\right)=0.6$} \\
\hline 1 & 178.50 & 179.79 & 0.72 \\
\hline 2 & 492.00 & 496.12 & 0.84 \\
\hline 3 & 963.00 & 976.20 & 1.37 \\
\hline \multicolumn{4}{|c|}{ Normalized Location of Crack, $\left(x_{c}\right)=0.8$} \\
\hline 1 & 178.80 & 180.24 & 0.81 \\
\hline 2 & 492.50 & 496.44 & 0.80 \\
\hline 3 & 964.00 & 972.99 & 0.93 \\
\hline
\end{tabular}

It is noticed that the results obtained are in good agreement with the results considered for comparison [27] and the errors are less than 1.5\%. Due to symmetry, the results for normalized positions of $0.2 \& 0.8$, and $0.4 \& 0.6$ are the same, as expected. The percentage error decreases as the normalized crack position moves towards the boundaries for third mode and second mode. While the percentage error in predicting the natural frequency increases for the first mode as the normalized crack location moves towards the clamped-clamped condition. 
Similarly, the natural frequencies for crack depth ratio of 0.4 at four different normalized crack positions are obtained and tabulated in Table 5-4.

Table 5-5: Natural Frequencies (Hz) of $C C$ Beam with Crack Depth Ratio, $\left(d_{c} / h\right)=0.4$

\begin{tabular}{|c|c|c|c|}
\hline Mode & $\underset{(\mathrm{Hz})}{\text { Afshari and Inman [27] }}$ & $\begin{array}{l}\text { AAF } \\
(\mathbf{H z})\end{array}$ & $\begin{array}{c}\% \\
\text { Error }\end{array}$ \\
\hline \multicolumn{4}{|c|}{ Normalized Location of Crack, $\left(x_{c}\right)=0.2$} \\
\hline 1 & 178.90 & 180.19 & 0.72 \\
\hline 2 & 490.00 & 493.41 & 0.70 \\
\hline 3 & 951.00 & 956.84 & 0.61 \\
\hline \multicolumn{4}{|c|}{ Normalized Location of Crack, $\left(x_{c}\right)=0.4$} \\
\hline 1 & 176.90 & 177.88 & 0.55 \\
\hline 2 & 488.50 & 491.85 & 0.69 \\
\hline 3 & 962.00 & 972.96 & 1.14 \\
\hline \multicolumn{4}{|c|}{ Normalized Location of Crack, $\left(x_{c}\right)=0.6$} \\
\hline 1 & 176.90 & 177.88 & 0.55 \\
\hline 2 & 488.50 & 491.85 & 0.69 \\
\hline 3 & 962.00 & 972.96 & 1.14 \\
\hline \multicolumn{4}{|c|}{ Normalized Location of Crack, $\left(x_{c}\right)=0.8$} \\
\hline 1 & 178.90 & 180.19 & 0.72 \\
\hline 2 & 490.00 & 493.41 & 0.70 \\
\hline 3 & 951.00 & 956.84 & 0.61 \\
\hline
\end{tabular}

In Table 5-5, it is observed the percentage error in predicting the natural frequency for the first and second mode is higher compared to the third mode as the location of the crack approaches the boundary condition. In the present study, the boundary penalty value for both uncracked and cracked beam is considered same. As the predicted natural frequency using $A A F$ approach is higher for the all three modes compared to Afshari and Inman [27], this indicated the selected boundary 
penalty value is higher and can be tuned by considering the effect of the crack. Validation carried out for $S S$ and $C C$ boundary conditions still yields an excellent result and is good agreement with reported literature. It indicates that crack as a penalty function model is well suited to accurately evaluate the natural frequencies for different boundary conditions. The selected boundary penalty value has scope for tuning by considering the effect of crack on the boundaries.

\subsubsection{Validation of a Shallow Crack in a Clamped-Free $(\mathrm{CF})$ Beam}

Validation for $C F$ boundary condition with a shallow crack is carried out and compared with results in Khnaijar and Benamar [18]. The geometrical and material properties used for validation are tabulated in Table 5-6.

Table 5-6: Geometrical Parameters and Material Properties Khnaijar and Benamar [18]

\begin{tabular}{|l|r|}
\hline Young's Modulus (E) & $70 \times 10^{9} \mathrm{~N} / \mathrm{m}^{2}$ \\
\hline Mass Density $(\rho)$ & $2700 \mathrm{~kg} / \mathrm{m}^{3}$ \\
\hline Length, $L$ & $0.8 \mathrm{~m}$ \\
\hline Thickness, $h$ & $0.006 \mathrm{~m}$ \\
\hline Width, $b$ & $0.05 \mathrm{~m}$ \\
\hline
\end{tabular}

The geometrical parameters and material properties tabulated in Table 5-6 are used to model a $C F$ beam. Using Eq. (4.12) for $N=12$, the penalty function is calculated as,

$$
\begin{aligned}
& k_{0}=k_{r 0}=\frac{E I}{L^{3}} \times 10^{5.49}=\frac{70 \times 10^{9} \times\left(0.05 \times 0.006^{3}\right)}{12 \times 0.8^{3}} \times 10^{5.49} \\
& k_{0}=k_{r 0}=3.8054 \times 10^{7}=1 \times 10^{7.58}
\end{aligned}
$$

Upon substituting the value of $k_{0}$ in Eq. (4.7), the $K_{P, C F}$ matrix is evaluated. Using Eq. (5.10), effective stiffness matrix $K_{E}$ of the beam with a shallow crack for the present system parameters are evaluated. On substituting the expression for $K_{P, C F}, K_{E}$ and $M$ in Eq. (5.11), the natural frequencies of the $C F$ beam with shallow crack are calculated. Khnaijar and Benamar [18] reported first two natural frequencies and Table 5-7 tabulates the first two natural frequencies estimated 
using $A A F$ approach of a $C F$ beam with shallow crack for normalized crack positions of $0.25,0.50$, and 0.75 from the fixed end, with a crack depth ratio of 0.5 .

Table 5-7: Natural Frequencies $(\mathrm{Hz})$ of $C F$ for $\left(d_{c} / h\right)=0.5$

\begin{tabular}{|c|c|c|c|}
\hline Mode & $\begin{array}{c}\text { Khnaijar and Benamar } \\
{[\mathbf{1 8}]} \\
(\mathbf{H z})\end{array}$ & $\begin{array}{c}\text { AAF } \\
\mathbf{( H z )}\end{array}$ & $\begin{array}{c}\text { \% } \\
\text { Error }\end{array}$ \\
\hline Normalized Crack Position $\left(x_{c}\right)=0.25$ \\
\hline 1 & 7.65 & 7.48 & 2.18 \\
\hline 2 & 48.52 & 48.29 & 0.48 \\
\hline \multicolumn{4}{|c|}{ Normalized Crack Position $\left(x_{c}\right)=0.50$} \\
\hline 1 & 7.71 & 7.65 & 0.77 \\
\hline 2 & 47.90 & 46.78 & 2.34 \\
\hline \multicolumn{4}{|c|}{ Normalized Crack Position $\left(x_{c}\right)=0.75$} \\
\hline 1 & 7.75 & 7.71 & 0.57 \\
\hline 2 & 48.28 & 47.74 & 1.12 \\
\hline
\end{tabular}

An increase in the percentage error in predicting the fundamental natural frequency as the position of the crack moves towards the fixed end is noticed. A similar trend is observed in the case of $S S$ and $C C$ boundary condition. The second natural frequency has the highest percentage error for the crack at mid point of the beam. This indicates a scope for improvement of boundary penalty values by considering the effect of crack.

Using the crack as a penalty model approach the results show an acceptable agreement; comparing with results from with Khnaijar and Benamar [18], there was less than 3\% error in predicting the natural frequency.

The effectiveness of the present approach is further validated with different crack modelling method proposed by Barad et.al.[20]. The geometrical and material properties are listed in Table $5-8$. 
Table 5-8: Geometrical Parameters and Material Properties Barad et.al., [20]

\begin{tabular}{|l|r|}
\hline Young's Modulus $(\mathrm{E})$ & $210 \times 10^{9} \mathrm{GPa}$ \\
\hline Mass Density $(\rho)$ & $7860 \mathrm{~kg} / \mathrm{m}^{3}$ \\
\hline Length, $L$ & $0.78 \mathrm{~m}$ \\
\hline Thickness, $h$ & $0.01 \mathrm{~m}$ \\
\hline Width, $b$ & $0.04 \mathrm{~m}$ \\
\hline
\end{tabular}

Using the geometrical parameters and material properties from Table 5-8, and using the Eq. (4.12) for $N=12$, the penalty function is calculated as,

$$
\begin{aligned}
& k_{0}=k_{r 0}=\frac{E I}{L^{3}} \times 10^{5.49}=\frac{210 \times 10^{9} \times\left(0.04 \times 0.01^{3}\right)}{12 \times 0.78^{3}} \times 10^{5.49} \\
& k_{0}=k_{r 0}=4.5584 \times 10^{8}=1 \times 10^{8.6}
\end{aligned}
$$

Upon substituting the value of $k_{0}$ in Eq. (4.7),the $K_{P, C F}$ matrix is evaluated.

Using Eq. (5.10), effective stiffness matrix $K_{E}$ of the beam with a shallow crack for the present system parameters are evaluated. On substituting the expression for $K_{P, C F}, K_{E}$ and $M$ in Eq. (5.11), the natural frequencies of the $C F$ beam with shallow crack are calculated. The first two natural modes are validated for four different normalized crack positions while keeping the crack depth ratio constant at 0.5 in Table 5-9. 
Table 5-9: Natural Frequencies $(\mathrm{Hz})$ of $C F$ for $\left(d_{\mathrm{c}} / h\right)=0.5$

\begin{tabular}{|c|c|c|c|}
\hline Mode & $\begin{array}{c}\text { Barad et.al., } \\
{[20]} \\
(\mathrm{Hz}) \\
\end{array}$ & $\begin{array}{c}\mathbf{A A F} \\
(\mathbf{H z})\end{array}$ & $\begin{array}{c}\% \\
\text { Error }\end{array}$ \\
\hline \multicolumn{4}{|c|}{ Normalized Crack Position $(\mathrm{xc})=0.2$} \\
\hline 1 & 13.02 & 12.76 & 1.99 \\
\hline 2 & 84.30 & 85.98 & 1.95 \\
\hline \multicolumn{4}{|c|}{ Normalized Crack Position $\left(x_{c}\right)=0.3$} \\
\hline 1 & 13.15 & 13.05 & 0.76 \\
\hline 2 & 83.30 & 84.91 & 1.89 \\
\hline \multicolumn{4}{|c|}{ Normalized Crack Position $\left(x_{c}\right)=0.4$} \\
\hline 1 & 13.28 & 13.31 & 0.22 \\
\hline 2 & 82.87 & 81.99 & 1.06 \\
\hline \multicolumn{4}{|c|}{ Normalized Crack Position $\left(x_{c}\right)=0.5$} \\
\hline 1 & 13.37 & 13.49 & 0.88 \\
\hline 2 & 81.82 & 80.37 & 1.77 \\
\hline
\end{tabular}

It is noticed that for the normalized crack location closer to the fixed end of the beam, the error in predicting the first natural frequency is higher. The same trend is observed in all the previous cases. This clearly indicates a scope for estimation of boundary penalty value based on the crack location. On the other hand, the results show an excellent agreement with less than $2 \%$ error in predicting the natural frequencies using crack as a penalty method approach. The present crack as a penalty approach provides flexibility in relocating the crack without the need to reformulate the whole problem to estimate natural frequencies leading to a lower computational time. 


\subsubsection{Validation of Proposed Approach to Estimate Modeshapes}

Using Eq. (5.9), the modeshapes of a beam with a shallow crack are extracted for different location and crack depth ratio. The results obtained are validated with the available literature results for $S S$, $C C$, and $C F$.

The dashed lines indicate the modeshapes obtained via Alternative Admissible Functions $(A A F)$, and the solid lines indicate the modeshapes of using the uncracked beam system eigenfunctions as the assumed mode. The first mode is indicated by blue colour, the second mode is indicated by red, and black depicts the third mode of the beam. The above stated legend applies to Figure 5-4 to Figure 5-8. The $S S$ and $C C$ boundary conditions modeshapes are validated with reference model of Afshari and Inman approach [27], while the $C F$ boundary condition modeshapes are validated with Khnaijar and Benamar [18]. Figure 5-4, represents the modeshapes of a $S S$ beam with a shallow crack at different normalized location and crack depth ratio $\left(d_{c} / h\right)=0.2$.

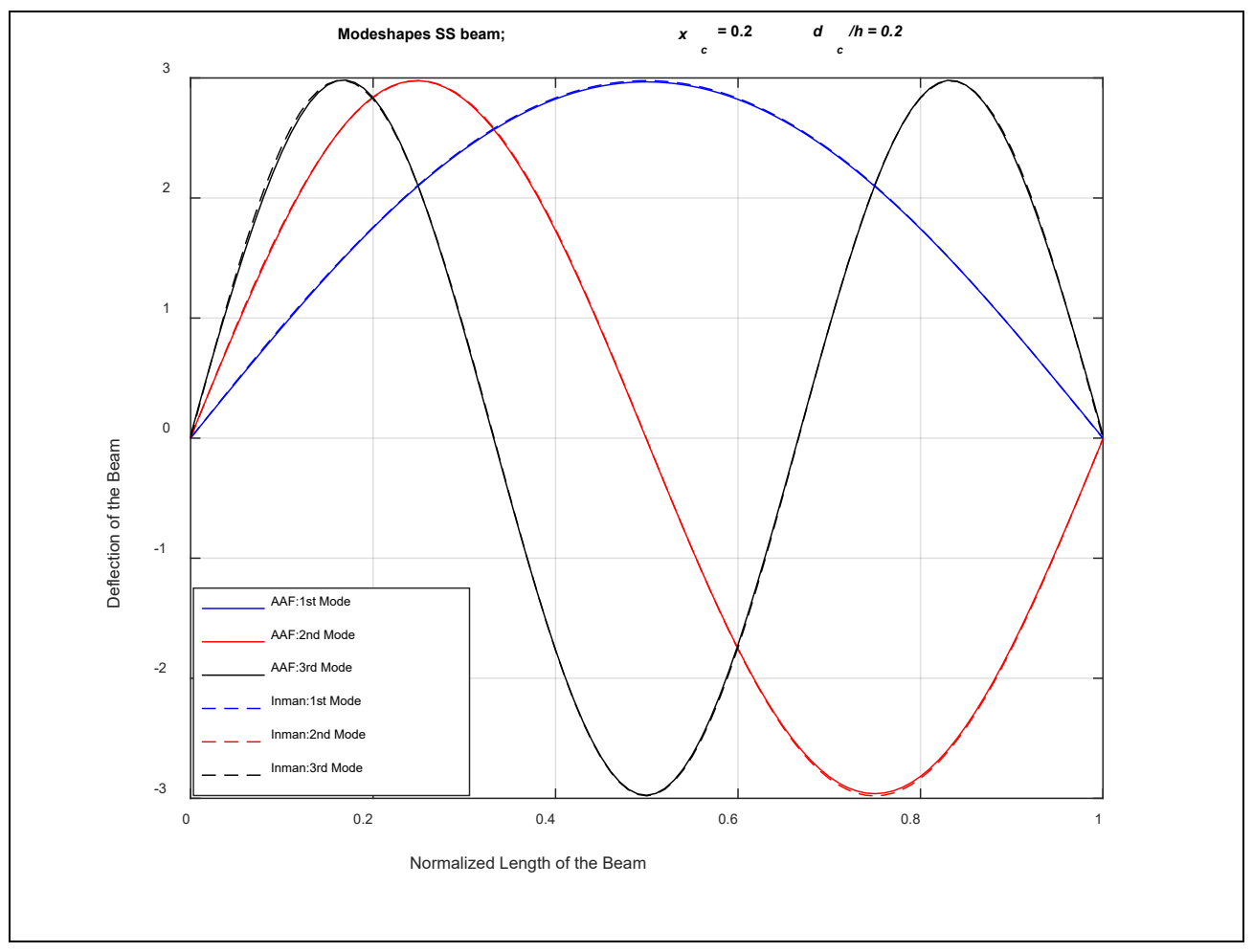

A 


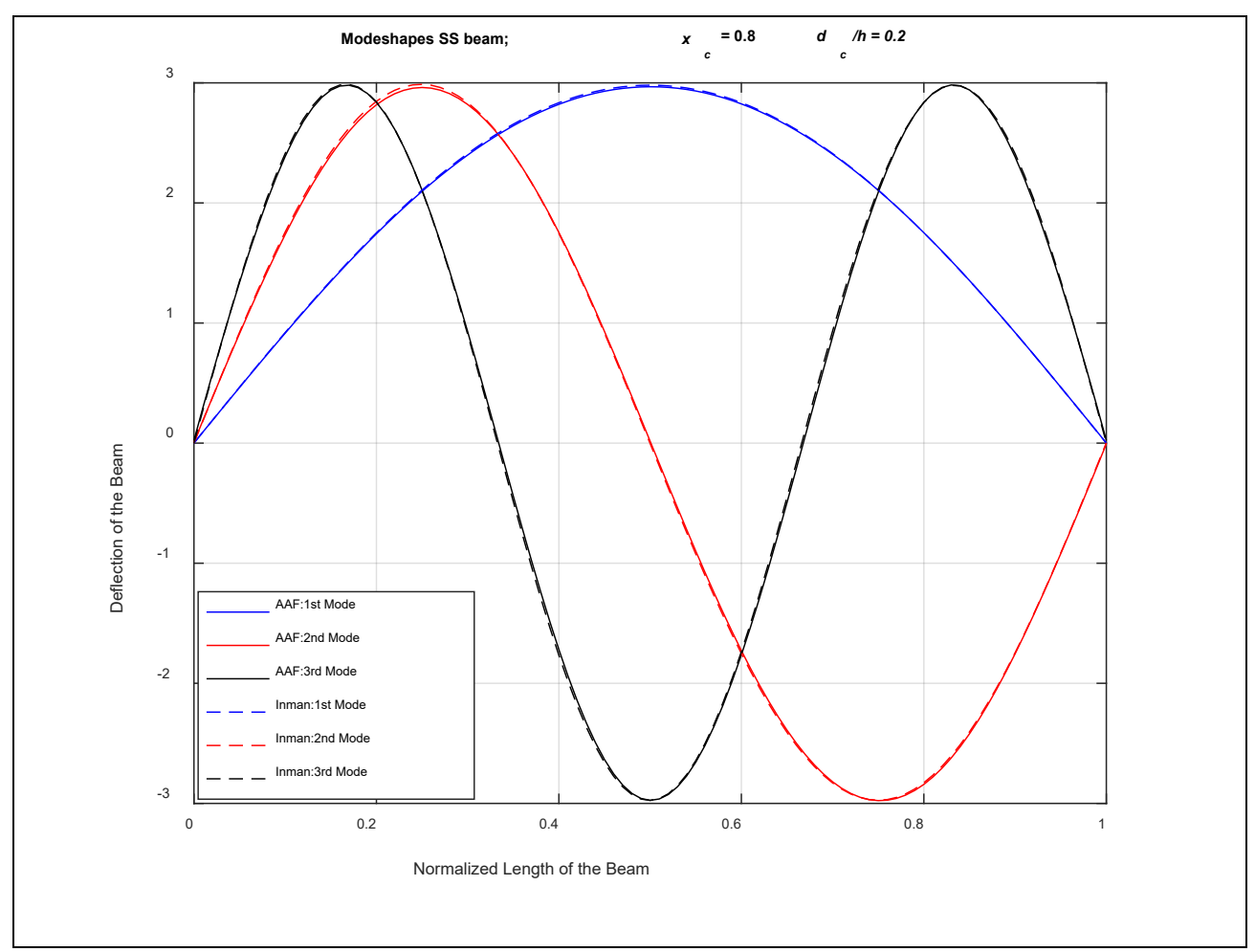

B

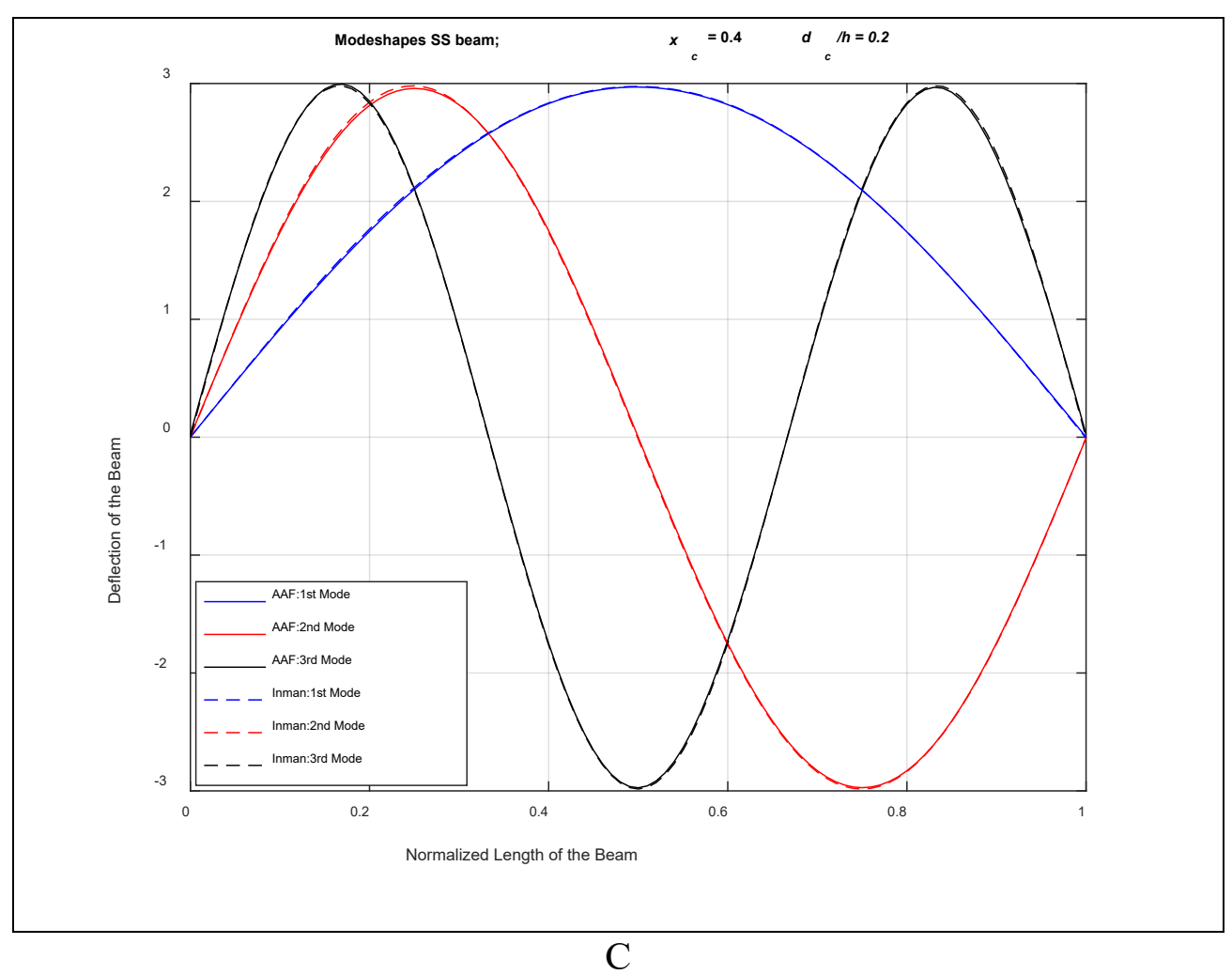




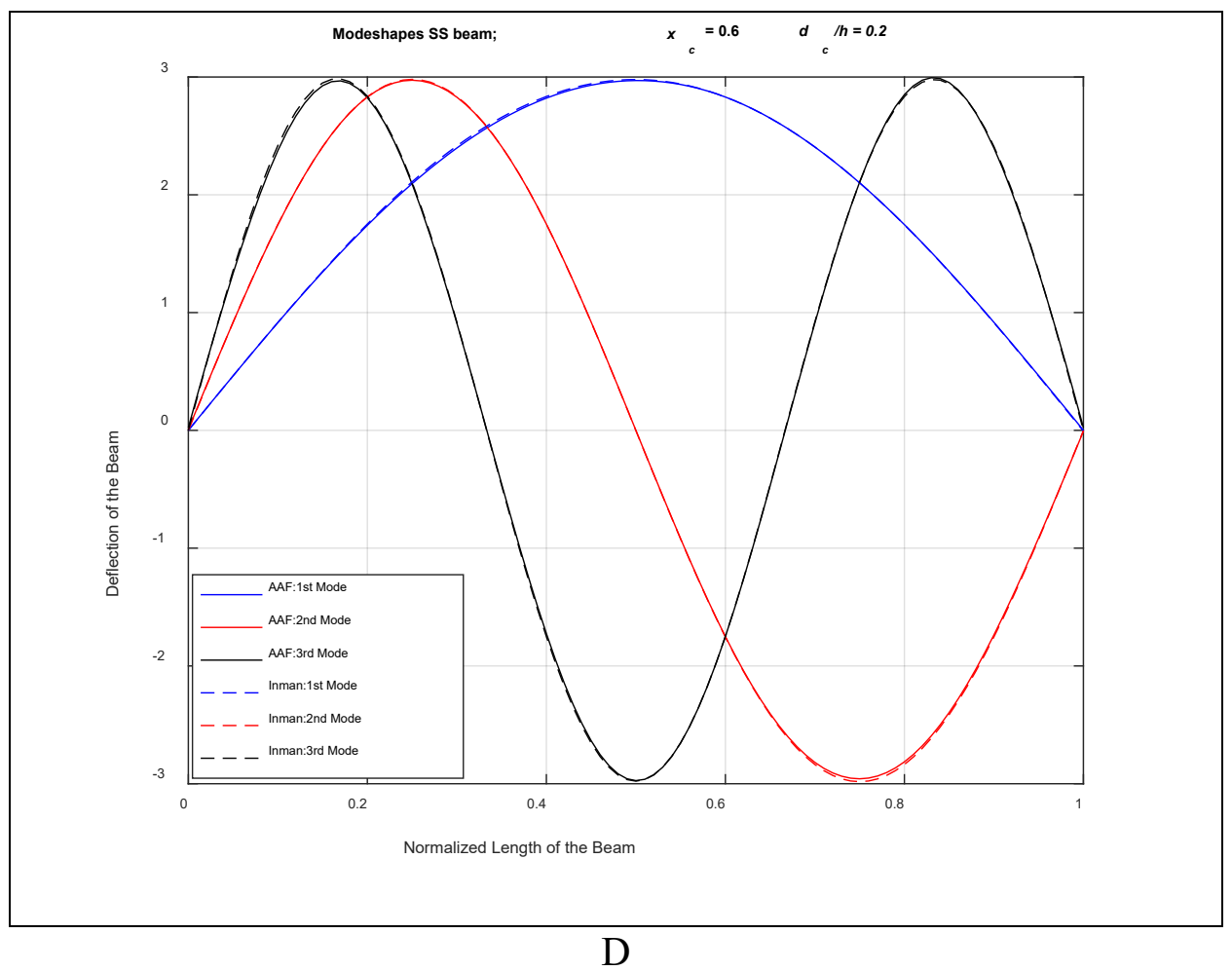

Figure 5-4: Modeshapes of SS Beam with Crack Depth Ratio, $(\mathrm{dc} / \mathrm{h})=0.2$. (A) Normalized Location of Crack, $(\mathrm{xc})=0.2$, (B) Normalized Location of Crack, $(\mathrm{xc})=0.8,(\mathrm{C})$ Normalized Location of Crack, $(\mathrm{xc})=0.4$, and $(\mathrm{D})$ Normalized Location of Crack, $(\mathrm{xc})=0.6$.

Figure 5-5,represents the modeshapes of a $S S$ beam with a shallow crack at different normalized location and crack depth ratio $\left(d_{c} / h\right)=0.4$. By visual examination of these plot, it is observed at the boundaries the lateral motion is restricted and the rotational motion is allowed to satisfy the $S S$ beam boundary condition. It is noticed that the present crack modelling approach used with $A A F$ method satisfies the boundary condition while generating the modeshapes for different normalized locations of crack and crack depth ratios. In Figure 5-4 and Figure 5-5 the modeshapes are in symmetry for normalized crack location of $0.2 \& 0.8$, and $0.4 \& 0.6$. 

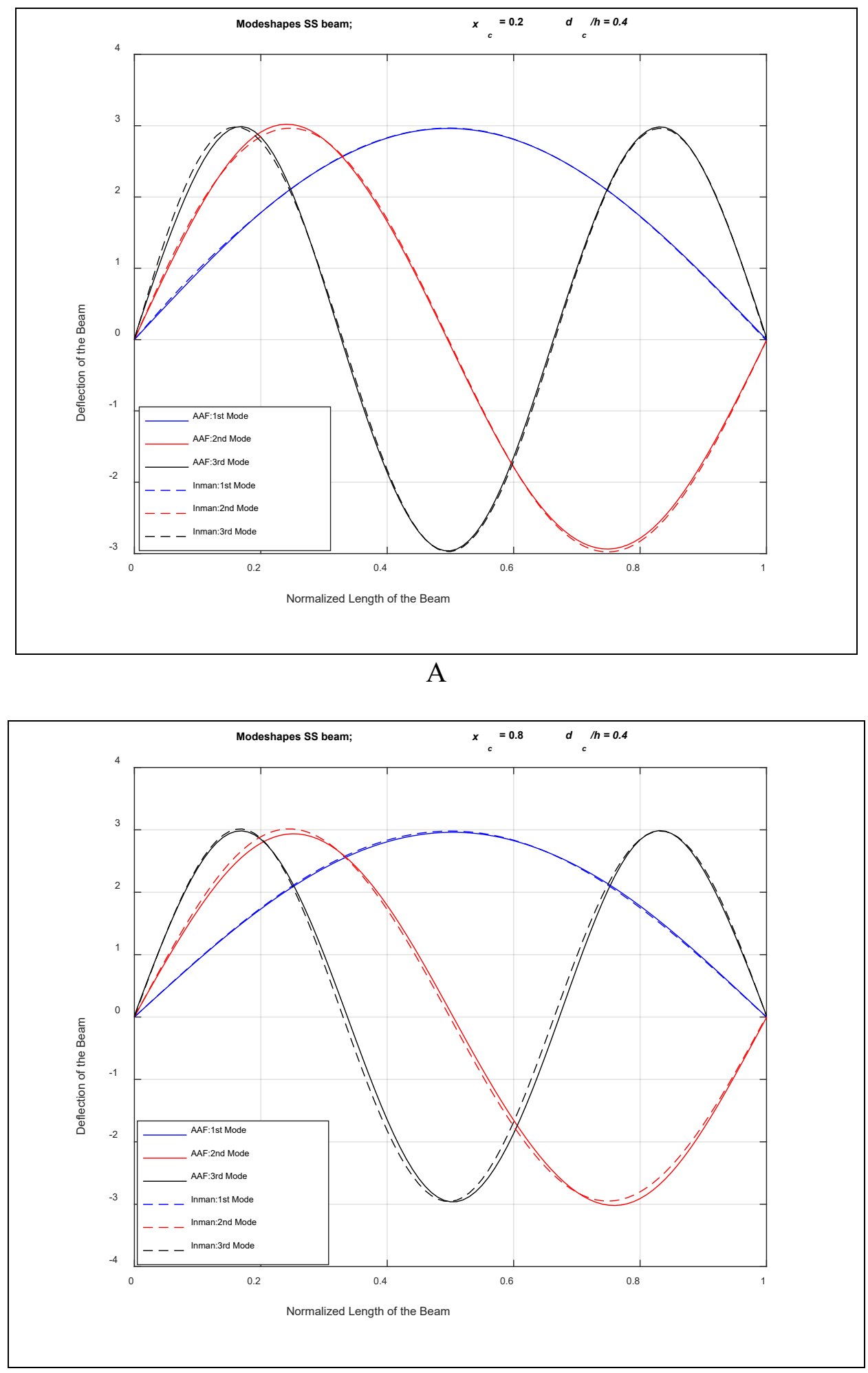

B 

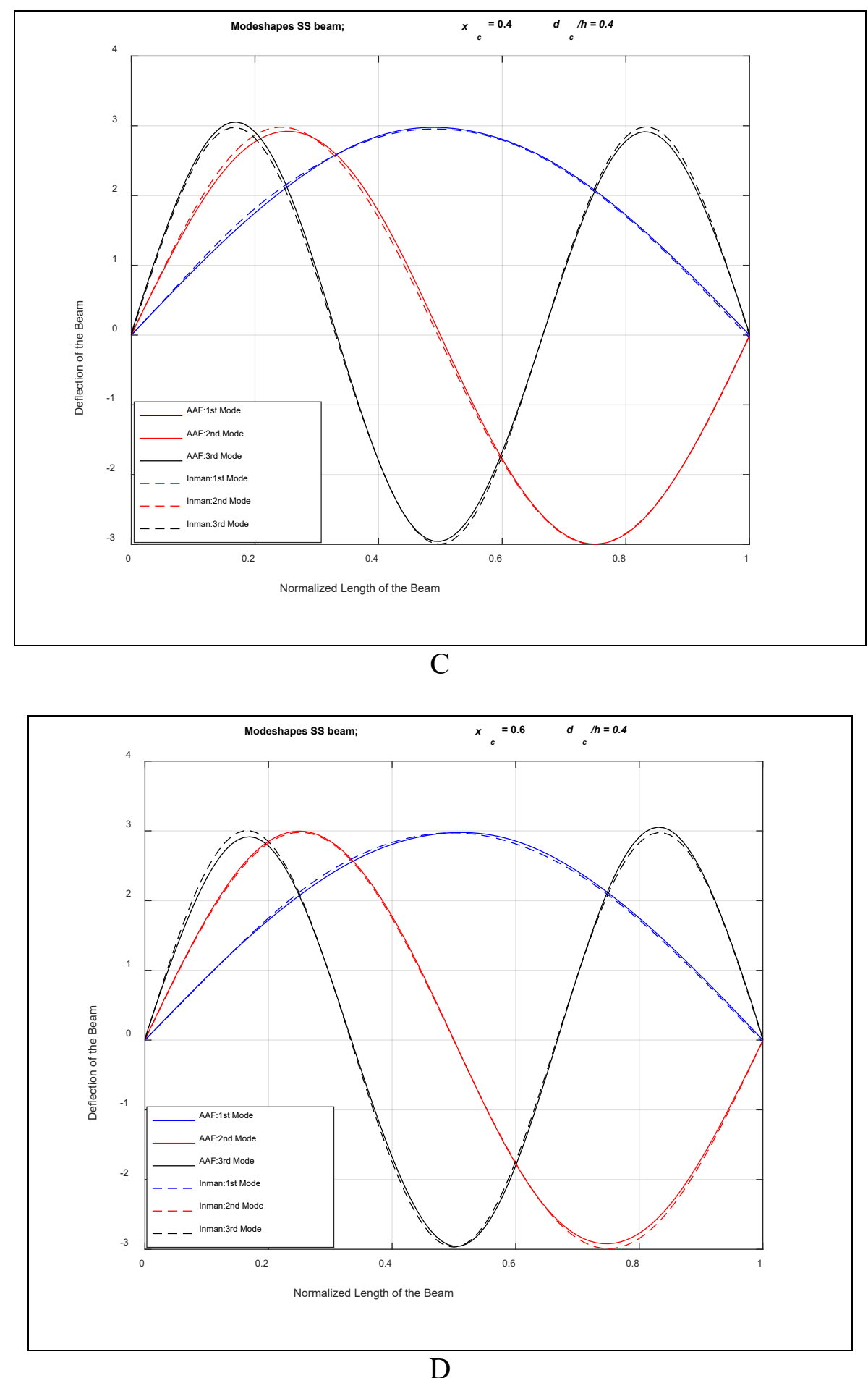

Figure 5-5: Modeshapes of SS Beam with Crack Depth Ratio, $\left(d_{c} / h\right)=0.4,(\mathrm{~A})$ Normalized Location of Crack, $\left(x_{c}\right)=0.2$, (B) Normalized Location of Crack, $\left(x_{c}\right)=0.8,(\mathrm{C})$ Normalized Location of Crack, $\left(x_{c}\right)=0.4$, and (D) Normalized Location of Crack, $\left(x_{c}\right)=0.6$ 
To validate the proposed approach for $C C$ beam with shallow crack, the modeshapes for different normalized location and crack depth ratio of 0.2 is depicted in Figure 5-6.

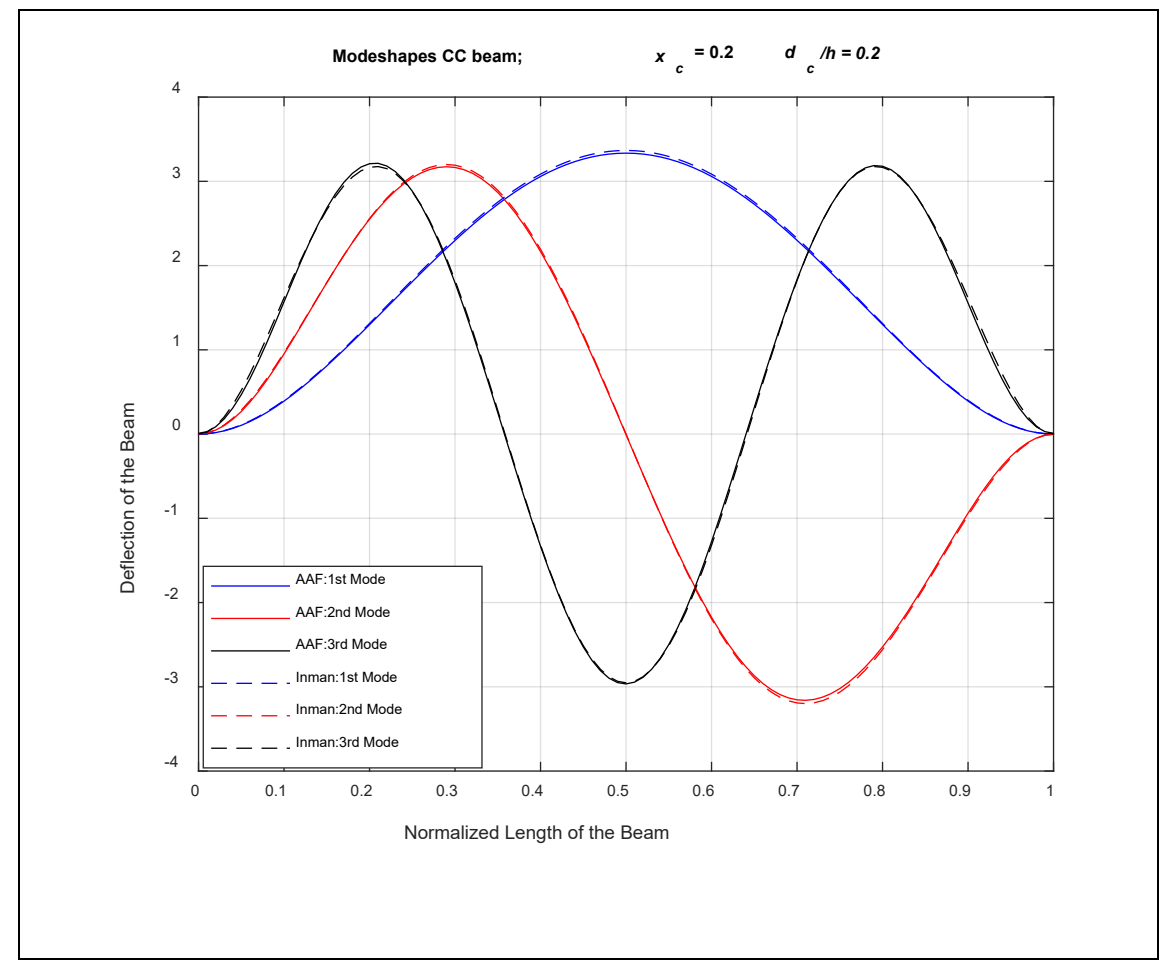

A

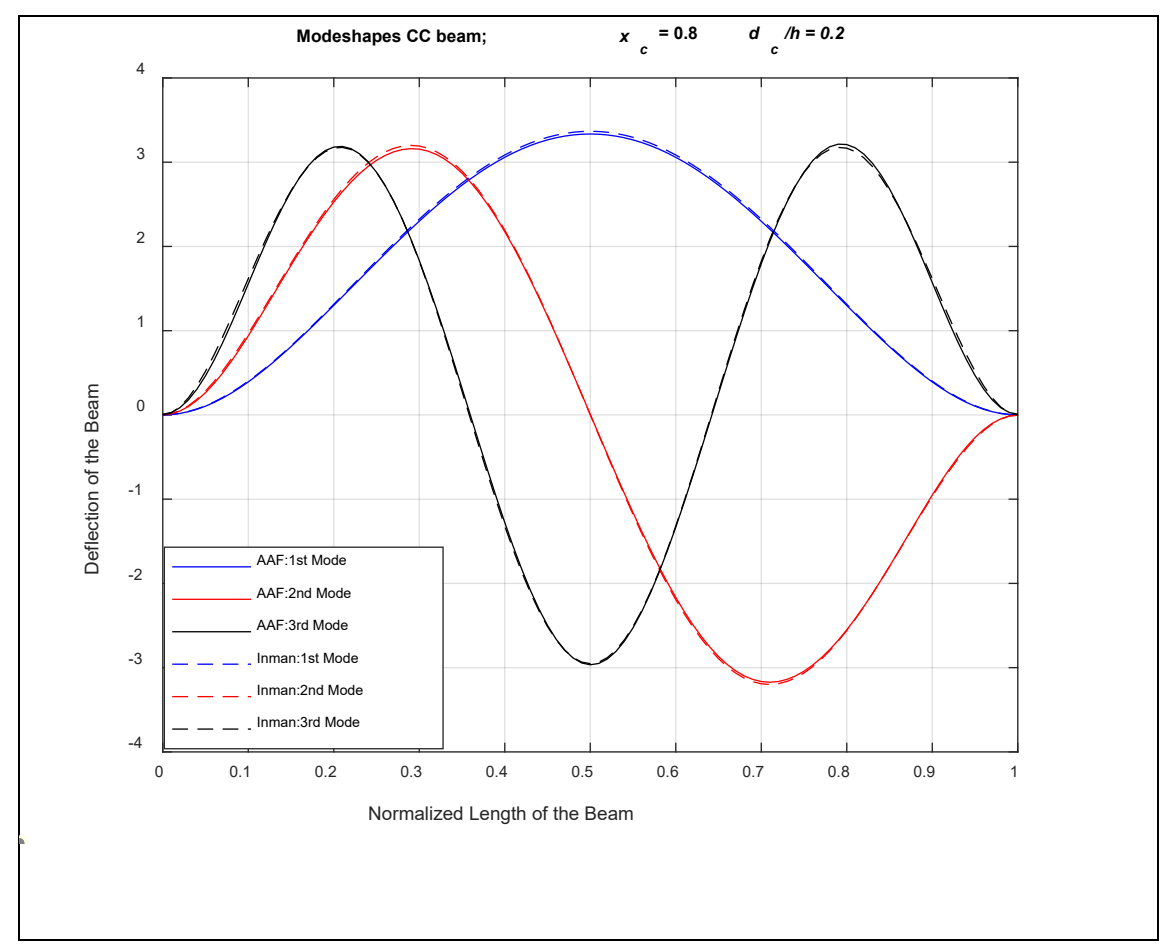

B 

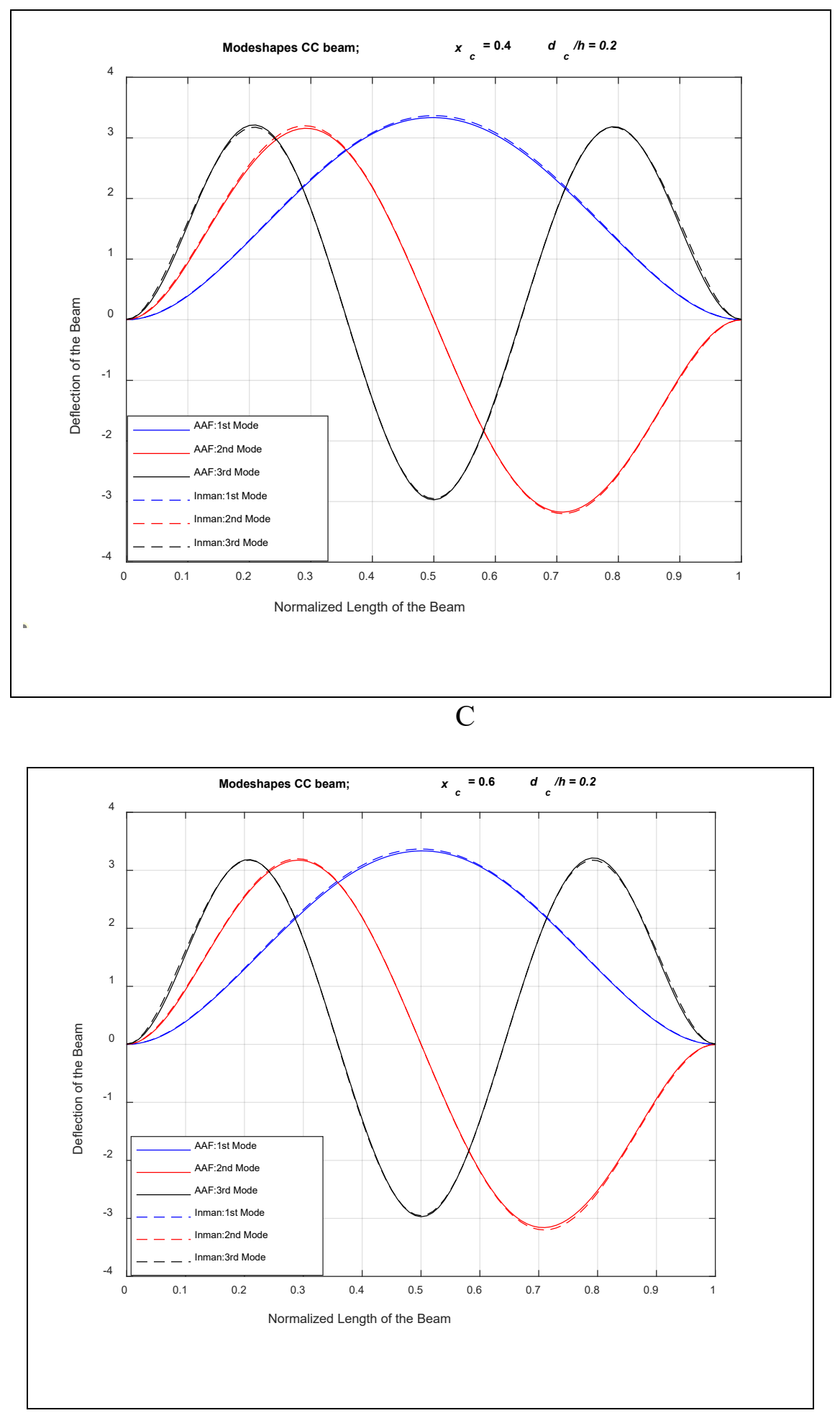

$\mathrm{D}$

Figure 5-6: Modeshapes of CC Beam with Crack Depth Ratio, $(d c / h)=0.2$, (A) Normalized Location of Crack, $(\mathrm{xc})=0.2$, (B) Normalized Location of Crack, $\left(x_{c}\right)=0.8$, (C) Normalized Location of Crack, $\left(x_{c}\right)=0.4$, and (D) Normalized Location of Crack, $\left(x_{c}\right)=0.6$ 
In Figure 5-6, it is noticed that the boundary conditions exhibited by the $C C$ boundary condition is satisfied by the proposed method. At the clamped end of the beam, the rotational and the lateral motion are restricted and are clearly depicted in the modeshapes. An excellent agreement with the reference model Afshari and Inman approach [27] is observed. Similarly, in Figure 5-7 modeshapes of a $C C$ beam for different normalized locations and crack depth ratio of 0.4 is derived and plotted.

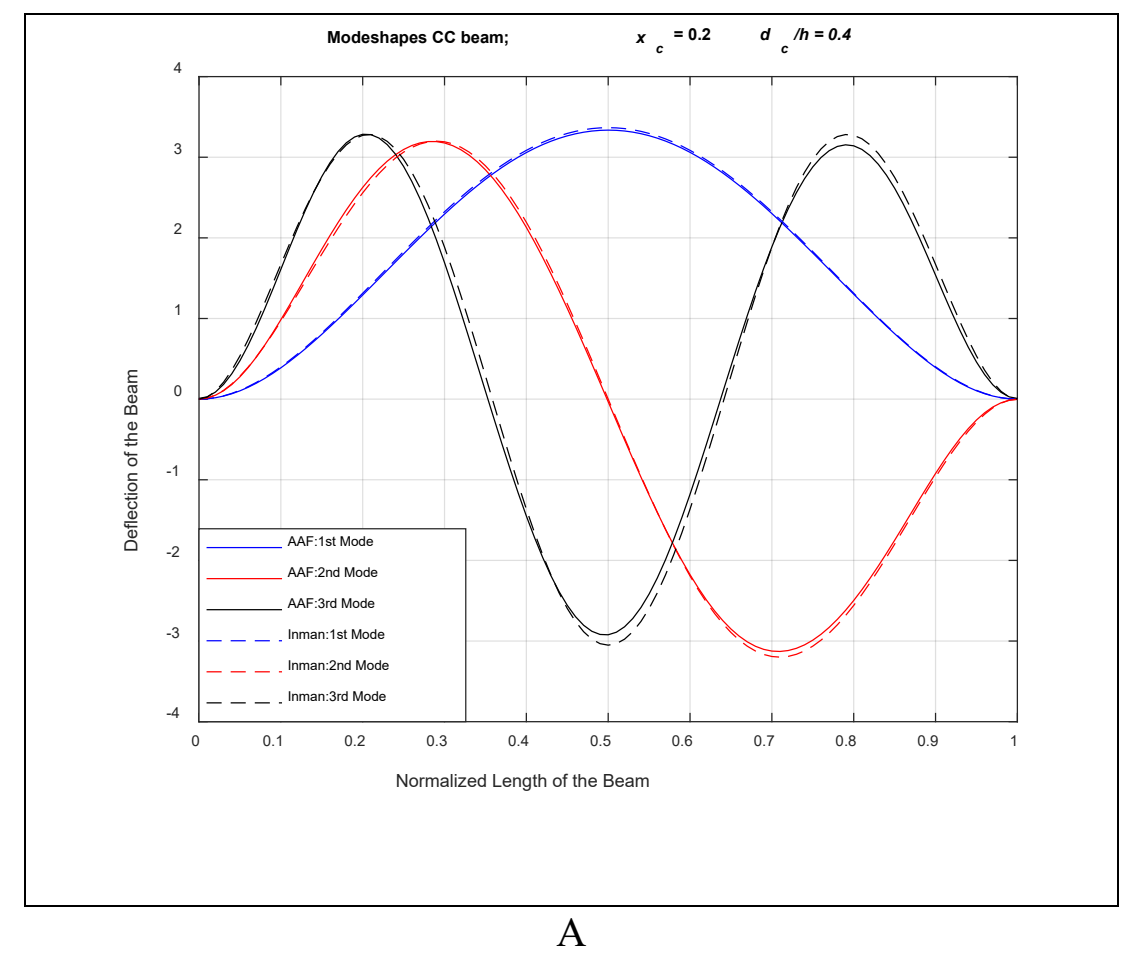




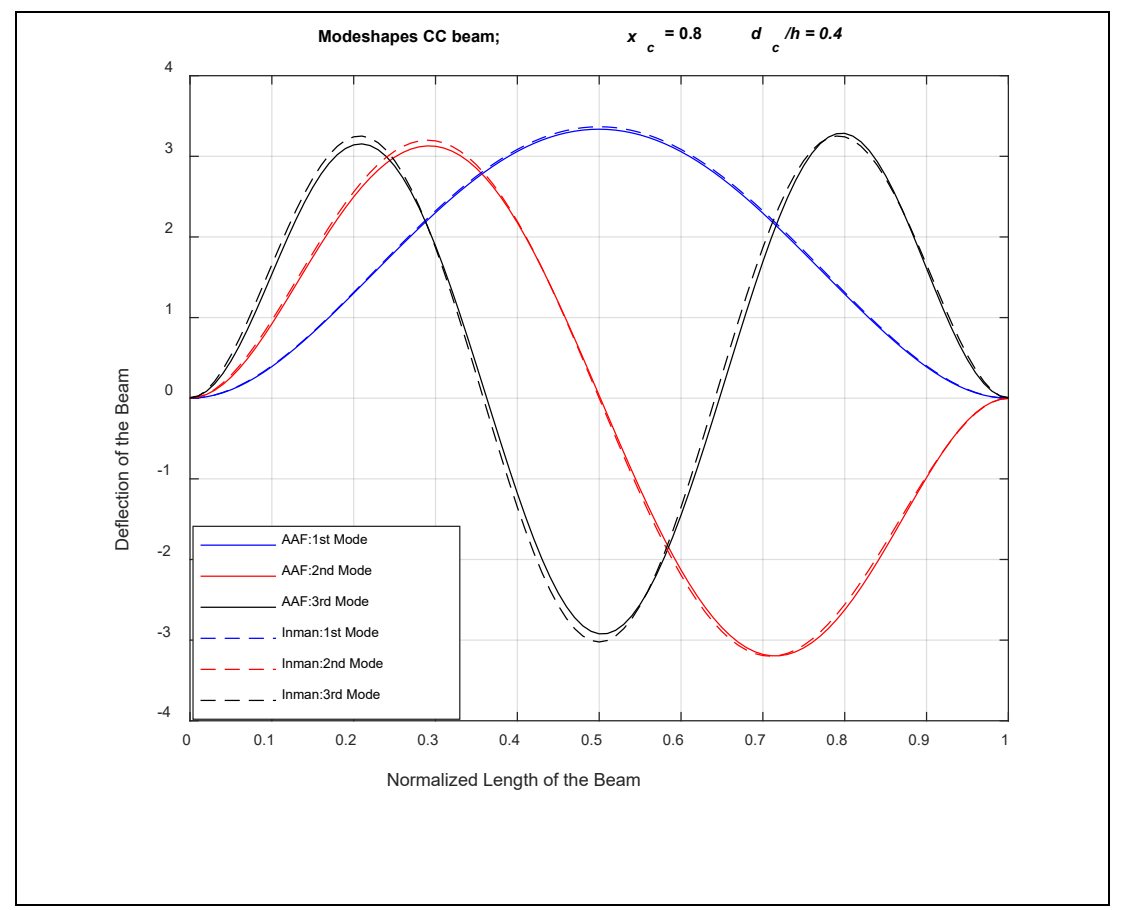

B

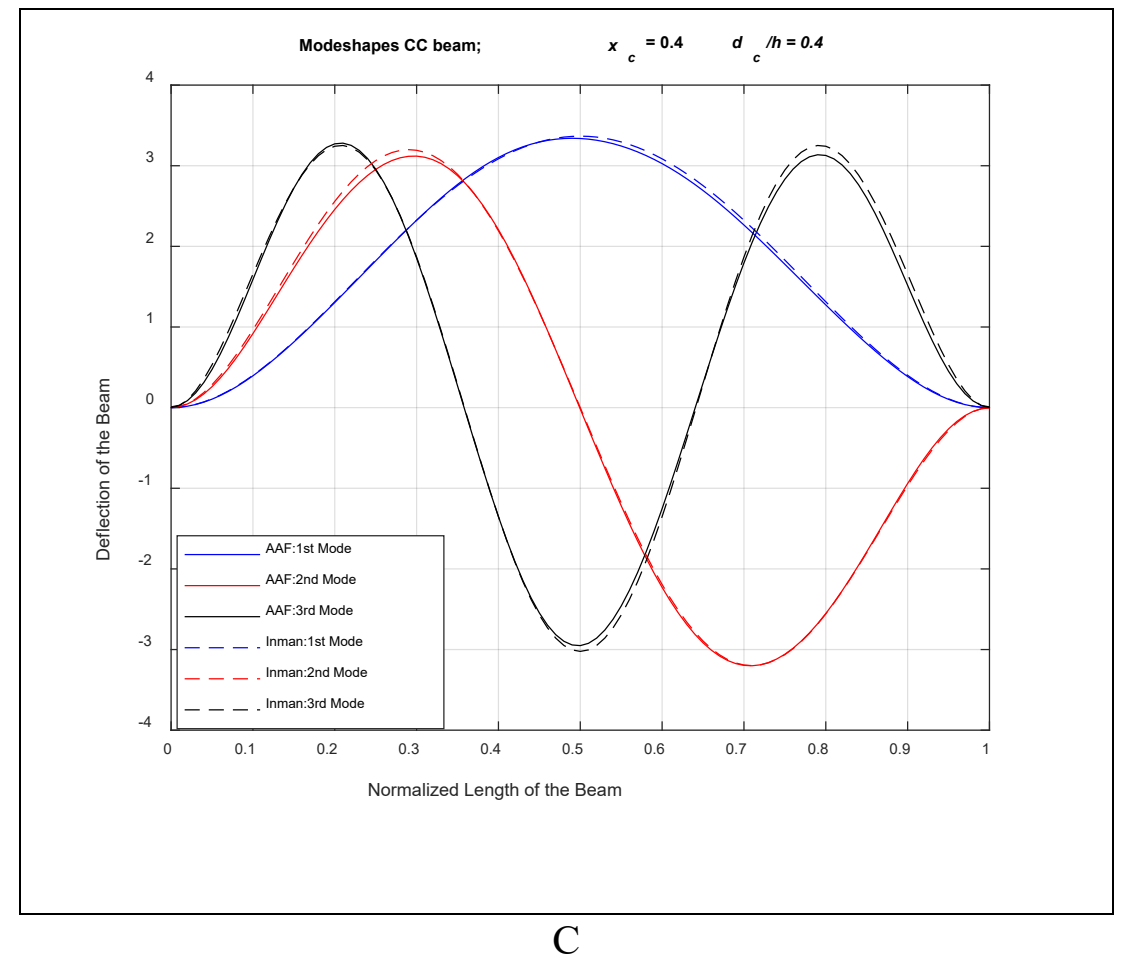




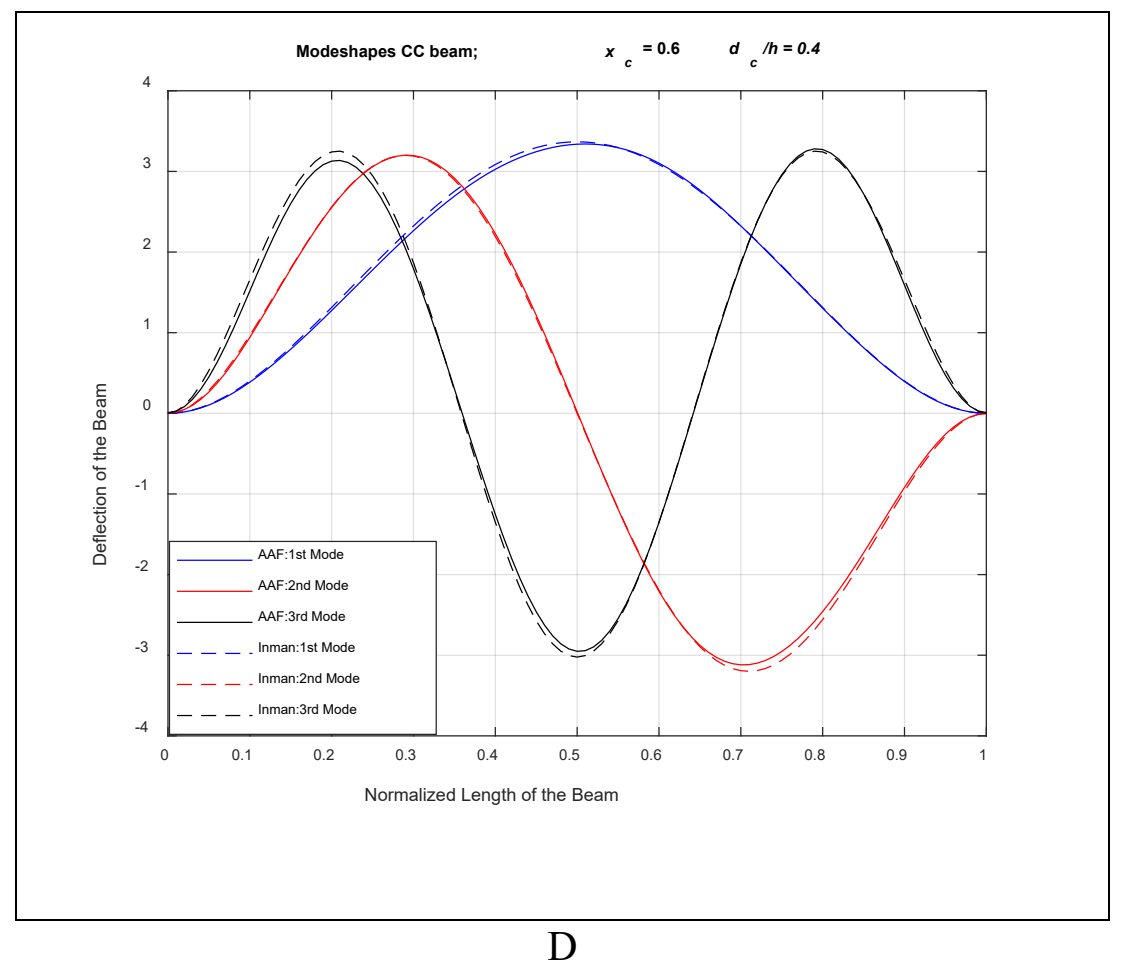

Figure 5-7: Modeshapes of $C C$ Beam with Crack Depth Ratio, $\left(d_{c} / h\right)=0.4$. (A) Normalized Location of Crack, (xc) $=0.2$, (B) Normalized Location of Crack, $\left(x_{c}\right)=0.8,(\mathrm{C})$ Normalized Location of Crack, $\left(x_{c}\right)=0.4$, and (D) Normalized Location of Crack, $\left(x_{c}\right)=0.6$

It is observed in Figure 5-7, the modes shapes have a mirror image for normalized crack location of $0.2 \& 0.8$, and $0.4 \& 0.6$. This indicates the selected set of $A A F$ with boundary penalties and crack model as penalty method produces the predicted behaviour for both the $S S$ and $C C$ boundary conditions. The proposed approach can be used to generate modeshapes for different crack locations and depth ratio efficiently.

The method is further validated for $C F$ boundary conditions with Khnaijar and Benamar [18]. Figure 5-8, the crack depth ratio is kept constant at 0.5 and the modeshape is derived for three normalized crack positions. 


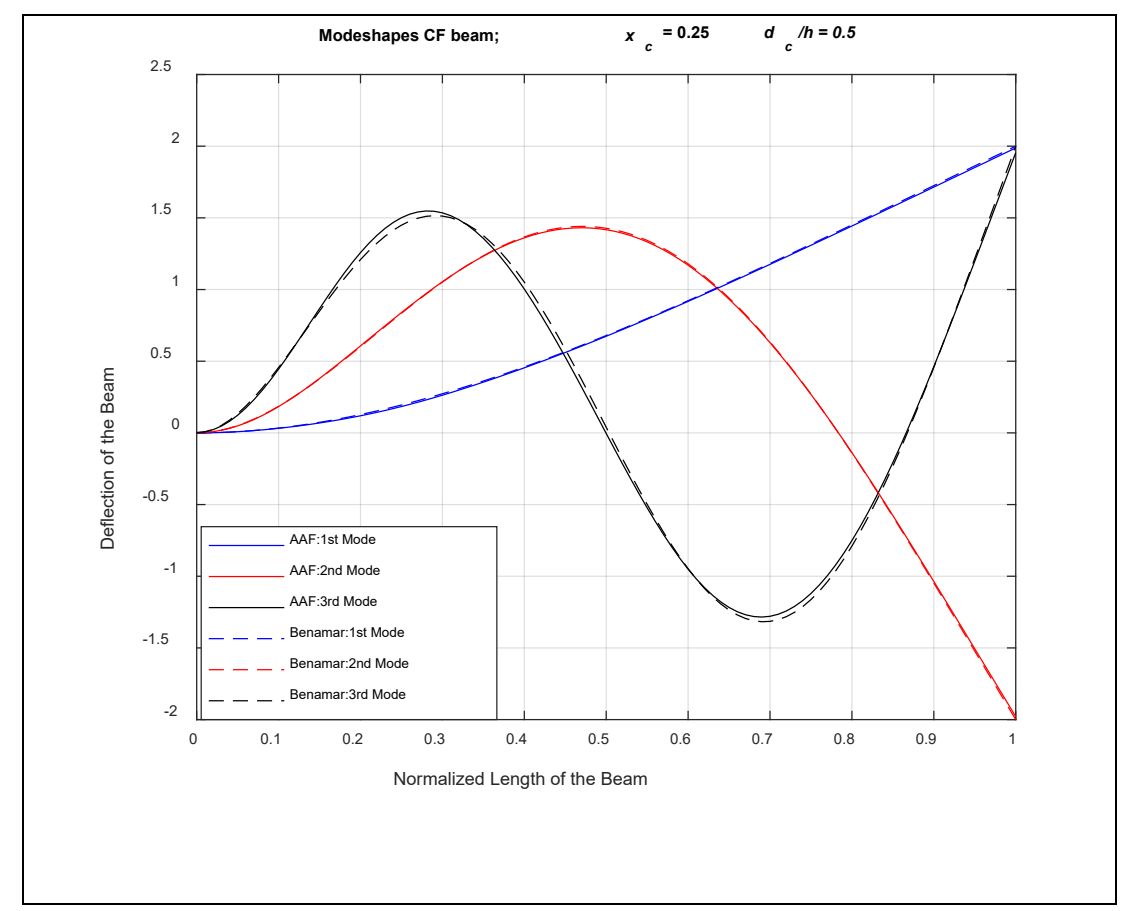

A

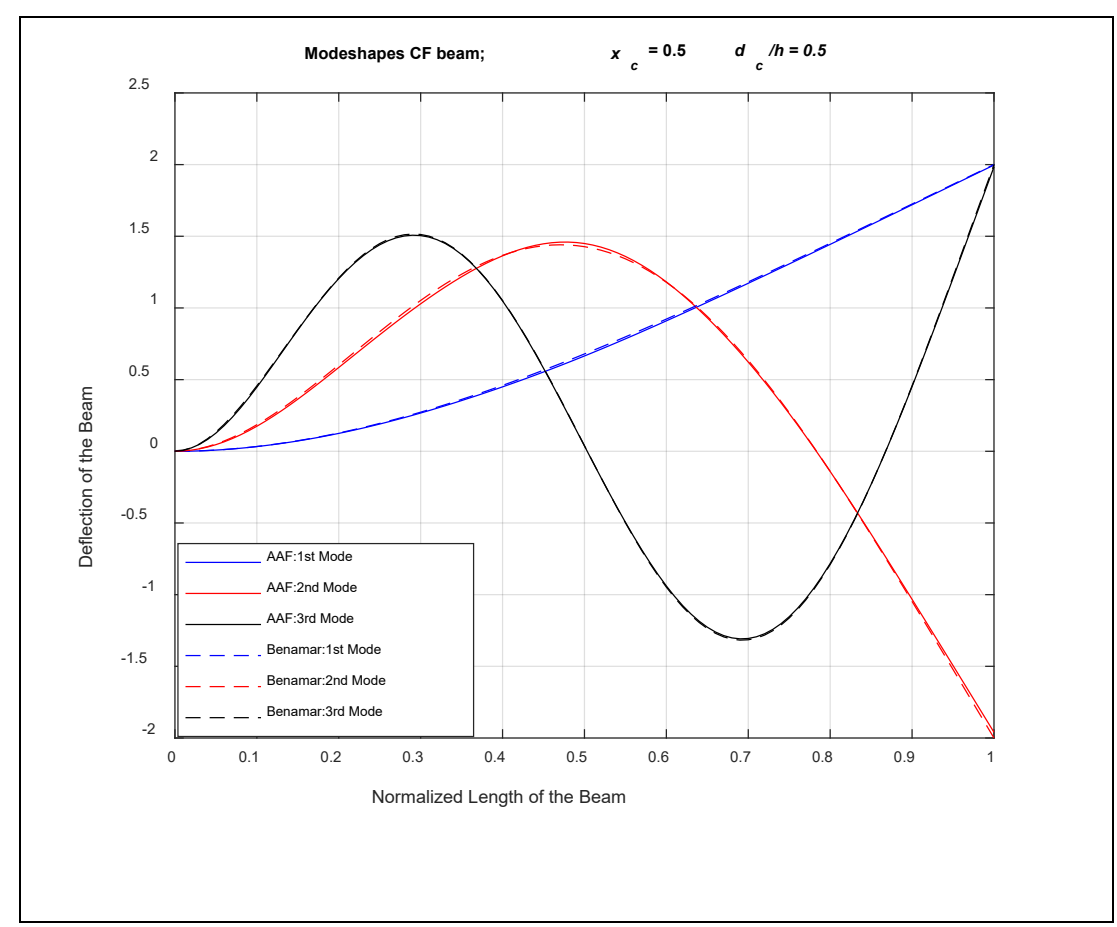

B 


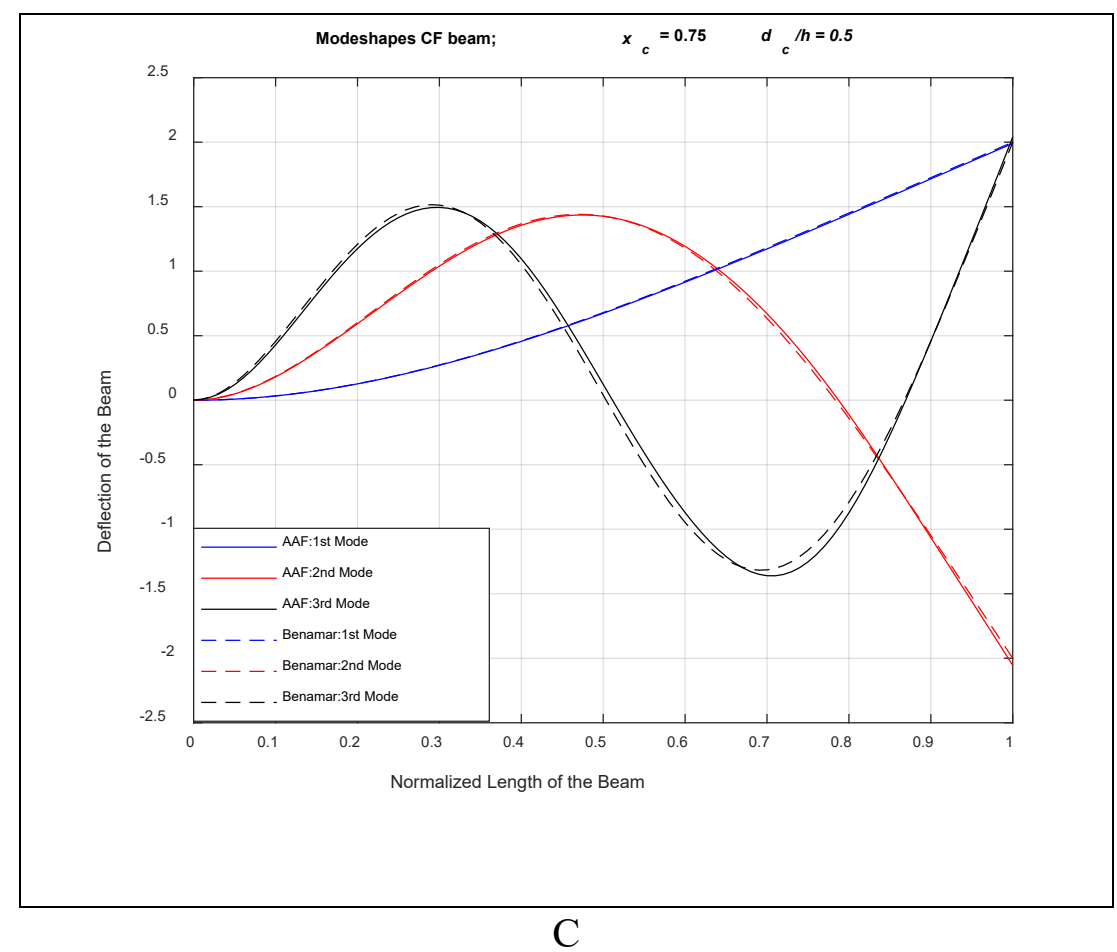

Figure 5-8: Modeshapes of $C F$ Beam with Crack Depth Ratio, $(d c / h)=0.5$. (A) Normalized Location of Crack, $\left(x_{c}\right)=0.25$, (B) Normalized Location of Crack, $\left(x_{c}\right)=$ 0.50 , and $(\mathrm{C})$ Normalized Location of Crack, $\left(x_{c}\right)=0.75$

Plots of the measured modeshapes for the first three natural frequencies of vibration for a crack depth 0.5 for normalized crack locations $0.25,0.5$, and 0.75 are plotted. The proposed method efficiently satisfies the different boundary conditions and can be used to derive modeshapes for various normalized shallow crack locations. 


\subsection{Conclusion}

A penalty method has been developed to model a shallow crack in a beam. In this method, the crack is modelled as a pair of virtual linear and rotational springs. The effect of the virtual linear spring is assumed to be negligible on the crack and the virtual rotational spring is modelled as a massless rotational spring. The loss of energy due to the presence of crack is accommodated by modifying the potential energy of the beam. Using this method, the dynamic characteristics of the beam with three different boundaries i.e., Simply-Supported (SS), Clamped-Clamped (CC) and Clamped-Free $(C F)$ boundary conditions are validated with previous literatures. The obtained results show good agreement with previous literatures. In the case of $S S$ and $C C$ boundary conditions, the obtained natural frequency values indicate a symmetry for normalized crack locations $0.2 \& 0.8$, and $0.4 \& 0.6$, as would be expected from the physical symmetry of the beam, even though not all the $A A F$ are symmetric about the centre of the beam. The percentage error in predicting natural frequencies using the crack modelling approach of Afshari and Inman [27] with $A A F$ method and crack as a penalty is less than $1 \%$ for Simply-Supported (SS) beam and less than $2 \%$ for Clamped-Clamped $(C C)$ beam. This indicates a scope for estimation of boundary penalty value based on the boundary condition and the crack. For the Clamped-Free boundary condition, the method is validated with results from Khnaijar \& Benamar [18], and Barad et.al. [20], the obtained results were in good agreement with the literature for crack depth ratio of 0.5 . It was observed that the percentage error in predicting the first natural frequency for all the three cases is higher compared to the other two modes as the location of the crack was moved closer to boundary condition. The percentage error in predicting the natural frequency was less than $3 \%$ compared to results in Khnaijar and Benamar [18] and less than 2\% compared to results in Barad et.al., [20]. The estimated natural frequencies obtained using the present crack modelling method prove to be 
efficient. The method was used to derive the modeshapes of the $S S, C C$, and $C F$ beam with a shallow crack. The method was validated with eigenvalues results obtained from previous literature for various crack depth and normalized crack depth ratios. It was observed that the proposed method satisfies the different boundary conditions and produces accurate modeshapes. By visual examination, the modeshapes are in excellent agreement with the reported literature.

Finally, the proposed method provides flexibility in choosing the location of crack and depth of crack by modelling it as a crack penalty function. The loss in energy due to the presence of a crack is calculated and accommodated by modifying the potential energy of the beam. The proposed expressions in Chapter 4 are utilized to calculate the boundary penalty function and provide excellent results for all three boundary conditions. The proposed method can be efficiently used to estimate accurate natural frequencies and modeshapes with ease of choosing the location of shallow cracks without the need to reformulate the problem for different boundary conditions or crack locations. 


\section{Conclusions and Future Work}

\subsection{Overview}

In the present work, the use of Alternative Admissible Functions $(A A F)$ as assumed modes to find the natural frequencies and modeshapes of a beam is considered. A set of Alternative Admissible Functions $(A A F)$, along with penalty functions, are used to obtain natural frequencies and modeshapes for an Euler-Bernoulli beam with various boundary conditions. It is observed the advantage of the $A A F$ method is that the choice of $A A F$ does not depend on the boundary conditions since the boundary conditions are implemented via penalty functions. Further, the work in this thesis aimed at proposing a methodology to estimate the value of penalty functions and establishing the relationship between the different boundary conditions, the underlying system parameters, and the choice of penalty. By using the proposed methodology, the dynamic characteristics of an Euler-Bernoulli uncracked beam were studied with the calculated penalty values.

The penalty function method was then extended to model a shallow crack as a massless rotational spring to determine the dynamic characteristics of a beam with a shallow crack. Three different boundary conditions were considered viz., Clamped-Free $(C F)$, Simply-Supported (SS) and Clamped-Clamped $(C C)$ throughout the study.

In Chapter 3, an overview on the Alternative Admissible Functions $(A A F)$ with penalties for an Euler-Bernoulli Beam $(E B B)$ with number of terms $N=12$ and the value of penalties as $k_{0}$ $=k_{\mathrm{r} 0}=1 \times 10^{10}$, based on reported literature. The present study is carried out for varying geometrical parameters and boundary conditions. The natural frequencies and the mode shapes obtained using the $A A F$ method with penalties were found to be in excellent agreement with the benchmark closed-form Euler-Bernoulli Beam values, with less than $1 \%$ error in predicting the 
natural frequency for $C F$ and $S S$ boundary conditions, and less than $2 \%$ error in predicting the natural frequency for $C C$ boundary condition.

It was observed in Chapter 3 that the selected values of penalties need to agree with the underlying beam properties, as well as the boundary conditions of the beam. This provided scope for appropriate selection of the penalty values depending on the boundary conditions and geometrical beam properties. In Chapter 4, a procedure was established to obtain a relationship between the different boundary conditions and the underlying system parameters. It was noticed that the specific value required for penalty function is directly proportional to the Young's modulus $(E)$ of the beam material, second moment of area of the cross section $(I)$, and inversely proportional the length $(L)$ of the beam under consideration. Following are the established relation between the boundary condition and the penalty functions.

\section{Boundary Conditions}

\section{Penalty function}

$$
\begin{array}{ll}
\text { Clamped-Free }(C F) & k_{0}=k_{r 0}=\frac{E I}{L^{3}} \times 10^{5.49} \\
\text { Simply-Supported }(S S) & k_{0}=k_{r 0}=\frac{E I}{L^{3}} \times 10^{5.03} \\
\text { Clamped-Clamped }(C C) & k_{0}=k_{r 0}=\frac{E I}{L^{3}} \times 10^{5.78}
\end{array}
$$

The proposed relationship is used to determine the frequencies and is validated for an Euler-Bernoulli beam for $C F, S S$ and $C C$ boundary conditions. The natural frequencies obtained using the $A A F$ method with estimated penalties were in excellent agreement with the benchmark closed-form Euler-Bernoulli values when compared to the penalty values selected from previous literature. The proposed relationship can be used with the three classical boundary conditions as the chosen functions for the $A A F$ do not change with boundary conditions. By using the developed 
expression, one can easily calculate the penalty values required at the boundaries. A similar approach can be used to obtain penalty values for various other boundary conditions.

Keeping in mind the benefits of the $A A F$ and penalty function, the proposed method is applied to beams with a shallow crack. In Chapter 5, an Euler-Bernoulli beam with shallow crack of crack depth ratio ranging from $0 \leq \frac{d_{c}}{h} \leq 0.4$ was considered. The penalty method was developed to model a shallow crack as a virtual massless rotational spring at the location of the crack. The $A A F$ provides the freedom to select any boundary condition and by using the proposed method in Chapter 4, the penalty function value is calculated for different boundary conditions. The crack modelled as a penalty function provided additional flexibility to relocate the crack and study the dynamic characteristics of the beam.

Using this method, the dynamic characteristics of the beam with a shallow crack for three different boundary conditions, $S S, C C$, and $C F$ were validated with reported literature. The obtained results demonstrated good agreement with previous literature. The percentage error in natural frequency using the Afshari and Inman approach [27] compared with $A A F$ method is less than $1 \%$ for a beam with $S S$ boundary condition and less than $2 \%$ for beam with $C C$ boundary condition. For a beam with a $C F$ boundary condition, the percentage error in natural frequency is less than 3\% compared with the results in Khnaijar and Benamar [18] and less than $2 \%$ compared with the results in Barad et.al., [20].

The proposed method was used to extract the modeshapes of different boundary conditions. Different crack depth and normalized crack locations were used to derive the modeshapes. Excellent agreement was found with modeshapes calculated using the uncracked beam eigenfunctions as the assumed modes. 
The proposed method shows excellent results for computing natural frequencies and modeshapes for different crack locations and crack depth ratios using the $A A F$ method with penalties. The methodology provides easy modification for changes in boundary conditions and crack parameters.

Publications the following publications were published or submitted:

- S. Kateel and N. Baddour, "Vibration Analysis of Beams using Alternative Admissible Functions with Penalties," in Volume 10: 33rd Conference on Mechanical Vibration and Sound (VIB), Virtual, Online, Aug. 2021, p. V010T10A025. doi: 10.1115/DETC2021-68459. [42]

- S. Kateel and N. Baddour, "Dynamic Characteristics of a Beam with a Shallow Crack using Alternative Admissible Functions", accepted for publication in Symmetry, January 2022 .

\subsection{Future work}

The results of the present study pave the way for condition monitoring for more complex structures and studying vibrations of combined dynamical systems. To increase the accuracy of the model with penalties, the values for the virtual linear and rotational springs can be calculated individually. The $A F F$ method with penalties provides a simple method to adapt to changes in boundary conditions, making way to study other structures using analytical methods. The proposed method can be further extended to study the effect of the number of terms, $N$ on the penalties.

The crack model as penalties provides scope to change the location and depth of crack and to conduct an in-depth parametric study. The effect of a crack on boundary penalties can be studied based on the location of the crack and depth of the crack. Experimental results to determine the dynamic characteristics of both beams with and without cracks would be an asset. 


\section{References}

[1] L. Meirovitch, Fundamentals of vibrations. Boston: McGraw-Hill, 2001.

[2] L. E. Monterrubio and S. Ilanko, "Sets of admissible functions for the Rayleigh-Ritz Method," Dubrovnik, Croatia, p. 97. doi: 10.4203/ccp.99.97.

[3] L. E. Monterrubio and S. Ilanko, "Proof of convergence for a set of admissible functions for the Rayleigh-Ritz analysis of beams and plates and shells of rectangular planform," Computers \& Structures, vol. 147, pp. 236-243, Jan. 2015, doi: 10.1016/j.compstruc.2014.09.008.

[4] R. E. Brown and M. A. Stone, "On the use of polynomial series with the Rayleigh-Ritz method," Composite Structures, vol. 39, no. 3-4, pp. 191-196, Nov. 1997, doi: 10.1016/S02638223(97)00113-X.

[5] D. J. Gorman and S. D. Yu, "A review of the superposition method for computing free vibration eigenvalues of elastic structures," Computers \& Structures, vol. 104-105, pp. 27-37, Aug. 2012, doi: 10.1016/j.compstruc.2012.02.018.

[6] C. S. Kim, P. G. Young, and S. M. Dickinson, "On the flexural vibration of rectangular plates approached by using simple polynomials in the Rayleigh-Ritz method," Journal of Sound and Vibration, vol. 143, no. 3, pp. 379-394, Dec. 1990, doi: 10.1016/0022-460X(90)90730-N.

[7] R. B. Bhat, "Natural frequencies of rectangular plates using characteristic orthogonal polynomials in rayleigh-ritz method," Journal of Sound and Vibration, vol. 102, no. 4, pp. 493499, Oct. 1985, doi: 10.1016/S0022-460X(85)80109-7. 
[8] J. W. Jaworski and E. H. Dowell, "Free vibration of a cantilevered beam with multiple steps: Comparison of several theoretical methods with experiment," Journal of Sound and Vibration, vol. 312, no. 4-5, pp. 713-725, May 2008, doi: 10.1016/j.jsv.2007.11.010.

[9] W. L. Li, "Comparison of fourier sine and cosine series expansions for beams with arbitrary boundary conditions," Journal of Sound and Vibration, vol. 255, no. 1, pp. 185-194, Aug. 2002, doi: $10.1006 /$ jsvi.2001.4108.

[10] W. L. Li, "Free vibrations of beams with general boundary conditions," Journal of Sound and Vibration, vol. 237, no. 4, pp. 709-725, Nov. 2000, doi: 10.1006/jsvi.2000.3150.

[11] F. Mir Hosseini and N. Baddour, "Alternative Admissible Functions for Natural Frequencies and Modeshapes of a Beam with Lumped Attachments," Structures, vol. 10, pp. 5975, May 2017, doi: 10.1016/j.istruc.2017.01.001.

[12] H. Ahmadian, J. E. Mottershead, and M. I. Friswell, "Boundary condition identification by solving characteristic equations," Journal of Sound and Vibration, vol. 247, no. 5, pp. 755-763, Nov. 2001, doi: 10.1006/jsvi.2001.3708.

[13] G. Štimac Rončević, B. Rončević, A. Skoblar, and R. Žigulić, "Closed form solutions for frequency equation and mode shapes of elastically supported euler-bernoulli beams," Journal of Sound and Vibration, vol. 457, pp. 118-138, Sep. 2019, doi: 10.1016/j.jsv.2019.04.036.

[14] L. Majkut, "Identification of beam boundary conditions in ill-posed problem," p. 15.

[15] S. Chesne, "Identification of beam boundary conditions using displacement derivatives estimations," IFAC Proceedings Volumes, vol. 45, no. 16, pp. 416-421, Jul. 2012, doi: 10.3182/20120711-3-BE-2027.00020.

[16] Y. Tao, "Impacts of various boundary conditions on beam vibrations," MS, West Virginia University Libraries, 2015. doi: 10.33915/etd.6774. 
[17] S. W. Doebling, C. R. Farrar, M. B. Prime, and D. W. Shevitz, "Damage identification and health monitoring of structural and mechanical systems from changes in their vibration characteristics: A literature review," LA--13070-MS, 249299, May 1996. doi: 10.2172/249299.

[18] A. Khnaijar and R. Benamar, "A new model for beam crack detection and localization using a discrete model," Engineering Structures, vol. 150, pp. 221-230, Nov. 2017, doi: 10.1016/j.engstruct.2017.07.034.

[19] P. F. Rizos, N. Aspragathos, and A. D. Dimarogonas, "Identification of crack location and magnitude in a cantilever beam from the vibration modes," Journal of Sound and Vibration, vol. 138, no. 3, pp. 381-388, May 1990, doi: 10.1016/0022-460X(90)90593-O.

[20] K. H. Barad, D. S. Sharma, and V. Vyas, "Crack Detection in Cantilever Beam by Frequency based Method," Procedia Engineering, vol. 51, pp. 770-775, 2013, doi: 10.1016/j.proeng.2013.01.110.

[21] H.-I. Yoon, I.-S. Son, and S.-J. Ahn, "Free vibration analysis of Euler-Bernoulli beam with double cracks," J Mech Sci Technol, vol. 21, no. 3, pp. 476-485, Mar. 2007, doi: 10.1007/BF02916309.

[22] N. T. Khiem and L. K. Toan, "A novel method for crack detection in beam-like structures by measurements of natural frequencies," Journal of Sound and Vibration, vol. 333, no. 18, pp. 4084-4103, Sep. 2014, doi: 10.1016/j.jsv.2014.04.031.

[23] E. I. Shifrin and R. Ruotolo, "Natural frequencies of a beam with an arbitrary number of cracks," Journal of Sound and Vibration, vol. 222, no. 3, pp. 409-423, May 1999, doi: 10.1006/jsvi.1998.2083.

[24] J. Hu and R. Liang, "An integrated approach to detection of cracks using vibration characteristics," p. 13. 
[25] T. G. Chondros and A. D. Dimarogonas, "Identification of cracks in welded joints of complex structures," p. 8.

[26] R. Y. Liang, F. K. Choy, and J. Hu, "Detection of cracks in beam structures using measurements of natural frequencies," Journal of the Franklin Institute, vol. 328, no. 4, pp. 505518, Jan. 1991, doi: 10.1016/0016-0032(91)90023-V.

[27] M. Afshari and D. J. Inman, "Continuous crack modeling in piezoelectrically driven vibrations of an Euler-Bernoulli beam," Journal of Vibration and Control, vol. 19, no. 3, pp. 341355, Feb. 2013, doi: 10.1177/1077546312437803.

[28] H. P. Lee and T. Y. Ng, "Natural frequencies and modes for the flexural vibration of a cracked beam," Applied Acoustics, vol. 42, no. 2, pp. 151-163, 1994, doi: 10.1016/0003682X(94)90004-3.

[29] N. T. Khiem and T. V. Lien, "A simplified method for natural frequency analysis of a multiple cracked beam," Journal of Sound and Vibration, vol. 245, no. 4, pp. 737-751, Aug. 2001, doi: $10.1006 /$ jsvi.2001.3585.

[30] R. D. Adams, P. Cawley, C. J. Pye, and B. J. Stone, "A vibration technique for nondestructively assessing the integrity of structures," Journal of Mechanical Engineering Science, vol. 20, no. 2, pp.93-100, Apr. 1978, doi: 10.1243/JMES_JOUR_1978_020_016_02.

[31] J. Fernández-Sáez, L. Rubio, and C. Navarro, “Approximate calculation of the fundamental frequency for bending vibrations of cracked beams," Journal of Sound and Vibration, vol. 225, no. 2, pp. 345-352, Aug. 1999, doi: 10.1006/jsvi.1999.2251.

[32] J. Fernández-Sáez and C. Navarro, "Fundamental frequency of cracked beams in bending vibrations: an analytical approach,” Journal of Sound and Vibration, vol. 256, no. 1, pp. 17-31, Sep. 2002, doi: 10.1006/jsvi.2001.4197. 
[33] W. M. Ostachowicz and M. Krawczuk, "Analysis of the effect of cracks on the natural frequencies of a cantilever beam," Journal of Sound and Vibration, vol. 150, no. 2, pp. 191-201, Oct. 1991, doi: 10.1016/0022-460X(91)90615-Q.

[34] T. G. Chondros, A. D. Dimarogonas, and J. Yao, "A continuous cracked beam vibration theory," Journal of Sound and Vibration, vol. 215, no. 1, pp. 17-34, Aug. 1998, doi: 10.1006/jsvi.1998.1640.

[35] X. F. Yang, A. S. J. Swamidas, and R. Seshadri, "Crack identification in vibrating beams using the energy method," Journal of Sound and Vibration, vol. 244, no. 2, pp. 339-357, Jul. 2001, doi: $10.1006 /$ jsvi.2000.3498.

[36] G.-L. Qian, S.-N. Gu, and J.-S. Jiang, "The dynamic behaviour and crack detection of a beam with a crack," Journal of Sound and Vibration, vol. 138, no. 2, pp. 233-243, Apr. 1990, doi: 10.1016/0022-460X(90)90540-G.

[37] T.Y. Kam and T.Y. Lee, "Identification of crack size via an energy approach," p. 11.

[38] J. K. Sinha, M. I. Friswell, and S. Edwards, "Simplified models for the location of cracks in beam structures using measured vibration data," Journal of Sound and Vibration, vol. 251, no. 1, pp. 13-38, Mar. 2002, doi: 10.1006/jsvi.2001.3978.

[39] X. B. Lu, J. K. Liu, and Z. R. Lu, "A two-step approach for crack identification in beam," Journal of Sound and Vibration, vol. 332, no. 2, pp. 282-293, Jan. 2013, doi: 10.1016/j.jsv.2012.08.025.

[40] T. Y. Kam and T. Y. Lee, "Detection of cracks in structures using modal test data," p. 7.

[41] A. D. Dimarogonas, "Vibration of cracked structures: A state of the art review," Engineering Fracture Mechanics, vol. 55, no. 5, pp. 831-857, Nov. 1996, doi: 10.1016/00137944(94)00175-8. 
[42] S. Kateel and N. Baddour, "Vibration Analysis of Beams Using Alternative Admissible Functions With Penalties," in Volume 10: 33rd Conference on Mechanical Vibration and Sound (VIB), Virtual, Online, Aug. 2021, p. V010T10A025. doi: 10.1115/DETC2021-68459. 